SPhT-T06/037

\title{
Mixed correlation function and spectral curve for the 2-matrix model
}

\author{
M. Bergère ${ }^{1}$, B. Eynard ${ }^{2}$
}

\begin{abstract}
:
We compute the mixed correlation function in a way which involves only the orthogonal polynomials with degrees close to $n$, (in some sense like the Christoffel

Darboux theorem for non-mixed correlation functions). We also derive new representations for the differential systems satisfied by the biorthogonal polynomials, and we find new formulae for the spectral curve. In particular we prove the conjecture of M. Bertola, claiming that the spectral curve is the same curve which appears in the loop equations.
\end{abstract}

\section{Contents}

1 Introduction 1

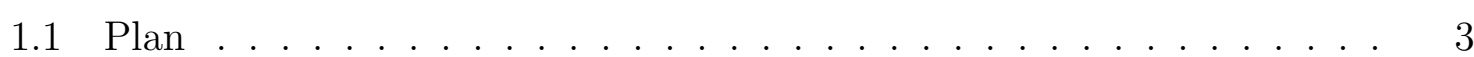

1.2 Main results . . . . . . . . . . . . . . . . . . . 4

2 Definitions and notations about bi-orthogonal polvnomials 5

2.1 Measure and integration paths . . . . . . . . . . . . . 5

2.2 Bi-orthogonal polvnomials . . . . . . . . . . . . . . . . . 7

2.2.1 Wave functions . . . . . . . . . . . . . . . 7

2.2.2 Cauchy transforms . . . . . . . . . . . . . . . . . 7

2.2.3 Fourier-Laplace transforms . . . . . . . . . . . . . . . . . 7

2.2.4 Third-tvpe functions ... . . . . . . . . . . . 8

2.3 Semi-infinite vectors and matrices . . . . . . . . . . . . . . . . 8

2.4 Recursion relations for the bi-orthogonal polvnomials . . . . . . . . . . 9

2.5 Relations between $\mathrm{Q}$ and $\mathrm{P}$................... 10

\footnotetext{
${ }^{1}$ E-mail: bergere@spht.saclay.cea.fr

${ }^{2}$ E-mail: eynard@spht.saclay.cea.fr
} 
2.6 Recursion relations for the Cauchy transforms . . . . . . . . . . . . 10

2.7 Recursion relations for the Fourier-Laplace transforms . . . . . . . . . . 10

2.8 Recursion relations for the third tvpe functions . . . . . . . . . . . . . 11

3 Inverses $\quad 11$

3.1 Inverse . . . . . . . . . . . . . . . . . . . . . . . . . . 11

3.2 Right inverse . . . . . . . . . . . . . . . . . . . . . . . . . 12

3.3 Left inverse . . . . . . . . . . . . . . . . . . . . 13

3.4 Relationship between right and left inverses . . . . . . . . . . . . . . 15

4 Kernels 16

4.1 Definition of the kernels . . . . . . . . . . . . . . . . 16

$4.1 .1 \quad K$ kernels . . . . . . . . . . . . . . . . . . 16

$4.1 .2 \quad J$ kernels . . . . . . . . . . . . . . . . . . . . . . 16

4.1.3 $H$ kernels . . . . . . . . . . . . . . . . . . . . . . . . . . 17

$4.1 .4 \quad \tilde{H}$ kernels . . . . . . . . . . . . . . . . . . . . 17

4.2 Christoffel-Darboux matrices . . . . . . . . . . . . . . 18

4.3 Christoffel-Darboux theorems . . . . . . . . . . . . . . . . . . 18

5 Determinantal formula for the orthogonal polvnomials and kernels 19

5.1 Determinantal formulae . . . . . . . . . . . . . . . . 19

5.2 Inverses . . . . . . . . . . . . . . . . . . . . . . . . . . 19

$\begin{array}{lll}6 & \text { Windows } & 20\end{array}$

7 Mixed correlation function $\quad 21$

7.1 Formula for $W_{n} \ldots \ldots \ldots \ldots$. . . . . . . . . . . . . . . 22

7.2 Recursion $W_{n+1}-W_{n} \ldots \ldots \ldots$. . . . . . . . . . . . . 23

8 Application: differential systems and spectral curve $\quad 24$

8.1 Differential svstems . . . . . . . . . . . . . . . . . . . . 24

8.2 Spectral curve . . . . . . . . . . . . . . . . . 25

9 Examples $\quad 27$

9.1 Gaussian case Ginibre polvnomials . . . . . . . . . . . . . . . . . . . 27

9.2 Gaussian elliptical case . . . . . . . . . . . . . . . . . . . . . . . . 27

10 Tau-function $\quad 28$

$\begin{array}{ll}11 \text { Conclusion } & 29\end{array}$ 
Appendix B Proof of determinantal formulae 33

B.1 Inverses . . . . . . . . . . . . . . . . . . . . 33

B.2 Determinants . . . . . . . . . . . . . . . . . 36

Appendix C Matrices $U$ and $\tilde{U}$

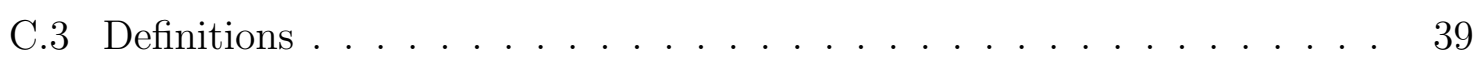

C.4 Some properties . . . . . . . . . . . . . . . . . . . . . 40

Appendix D $\quad$ Proof of theorem $7.1 \quad 42$

Appendix E Proof of theorem $7.3 \quad 45$

$\begin{array}{lll}\text { Appendix F } & \text { Proof of theorem 8.1 } & 48\end{array}$

F.5 U-conjecture, Lemma 8.1 . . . . . . . . . . . . . . . . . . 51

$\begin{array}{lll}\text { Appendix G } & \text { Proof of theorem } 8.3 & 51\end{array}$

$\begin{array}{lll}\text { Appendix H } & \text { Proof of theorem } 8.2 & 52\end{array}$

Appendix I A usefull formula for determinants with rank 2 matrices 53

\section{Introduction}

Consider a pair of random hermitean matrices $M_{1}$ and $M_{2}$, of size $n$, with the probability measure:

$$
\mathrm{e}^{-\operatorname{Tr}\left(V_{1}\left(M_{1}\right)+V_{2}\left(M_{2}\right)+M_{1} M_{2}\right)} d M_{1} d M_{2}
$$

This random 2-matrix model has many applications to physics (in particular in quantum gravity, i.e. statistical physics on a random surface and conformal field theory [30, 15]) and mathematics (bi-orthogonal polynomials [34]). Another important application of the 2-hermitean matrix model comes from the fact that it is the analytical continuation of the complex-matrix model, which describes the Dyson gaz at $\beta=2$ and is an important model of Laplacian growth [38. And the complex matrix model plays a crucial role in the AdS/CFT correspondance, in the so-called BMN limit [31. In that model, it is important to know how to compute mixed expectation values.

For all applications, one would like to be able to compute various expectation values. Some expectation values can be written in terms of eigenvalues of $M_{1}$ and $M_{2}$, 
for instance $<\operatorname{Tr} M_{1}^{k} \operatorname{Tr} M_{2}^{l}>$, which we call non-mixed because each trace contains only one type of matrix $M_{1}$ or $M_{2}$ but not both. On the contrary, mixed expectation values are those where $M_{1}$ and $M_{2}$ may appear together in the same trace, for instance $<\operatorname{Tr} M_{1}^{k} M_{2}^{l}>$. Mixed expectation values cannot be written in terms of eigenvalues of $M_{1}$ and $M_{2}$, and are thus more difficult to compute than non-mixed ones.

Beyond the technical challenge of computing them, mixed expectation values should play an important role in applications to boundary conformal field theory and to the BMN limit of string theory/CFT correspondance. While many formulae for non mixed expectation values have been known for a long time, in particular in terms of biorthogonal polynomials [16, 37, 23, 2, 3, 4, 1], it is only recently that formulae have been found for mixed traces. In particular the following expectation value:

$$
W_{n}(x, y)=1+\left\langle\operatorname{Tr} \frac{1}{x-M_{1}} \frac{1}{y-M_{2}}\right\rangle,
$$

was first computed in [8]. The idea was to diagonalize the hermitean matrices $M_{1}=$ $V X V^{\dagger}$ and $M_{2}=V U Y U^{\dagger} V^{\dagger}$ where $U$ and $V$ are unitary matrices and $X$ and $Y$ are diagonal matrices containing the eigenvalues of $M_{1}$ and $M_{2}$. Then:

$$
W_{n}(x, y)=1+\left\langle\operatorname{Tr} \frac{1}{x-X} U \frac{1}{y-Y} U^{\dagger}\right\rangle,
$$

and using the Morozov's formula [35, 19] for unitary integrals of the form $\left\langle U_{i j} U_{k l}^{\dagger}>\right.$, one reexpresses $W_{n}$ in terms of eigenvalues of $M_{1}$ and $M_{2}$ only. Then the integration over eigenvalues is done with the method of biorthogonal polynomials [33]. The result found in [8] is thus a $n \times n$ determinant involving recursion coefficients of bi-orthogonal polynomials:

$$
W_{n}(x, y)=\operatorname{det}_{n \times n}\left(\operatorname{Id}_{n}+\Pi_{n-1} \frac{1}{x-Q} \frac{1}{y-P^{t}} \Pi_{n-1}\right),
$$

where the matrices $Q$ and $P$ implement the recursion relation (multiplication by $x$ and $y$ ) of the biorthogonal polynomials, and $\Pi_{n-1}$ is the projection on the polynomials of degree $\leq n-1$. Those notations are explained in more details in section 2.4 ,

The mere existence of such a formula was a progress, but a $n \times n$ determinant is not convenient for practical computations and for taking large $n$ limits. In the nonmixed case, the Christoffel-Darboux theorem allows one to rewrite expectation values in terms of smaller determinants, whose size does not grow with $n$ [16, 3, 4].

The purpose of the present article is to find a similar property for $W_{n}(x, y)$, i.e. write it in terms of determinants whose size is independent of $n$.

As a byproduct of such a rewriting, we are able to find new formulae for the spectral curve of the isomonodromic differential system satisfied by the corresponding biorthogonal polynomials. 


\section{$1.1 \quad$ Plan}

- In section 2, we recall the definitions of bi-orthogonal polynomials, as well as their Fourier-Laplace transforms, Cauchy transforms, and the third type of solution introduced by [13, 25]. We also recall their recursion relations, obtained by multiplication by $x$ or derivation. We define the infinite matrices (finite band) $Q$ and $P$, which encode the recursion relations $(Q$ and $P$ are also Lax matrices).

- In section 3 , we study the inverse of $x-Q$ and $y-P$, in particular, we show that they have distinct right and left inverses. The difference between the right and left inverse, is related to the so-called folding matrix [1], and is found to be a sum of bi-orthogonal polynomials and their various transforms.

- In section 4, we introduce the kernels [16], which are the building blocks of correlation functions. We show that they satisfy some Christoffel Darboux relations.

- In section 5, we compute the biorthogonal polynomials, their Cauchy transforms, and the kernels as determinants involving matrices $Q$ and $P$.

- In section 6, we introduce the notion of windows of consecutive bi-orthogonal polynomials [9, 10, 11, because the recursion relations (matrix $Q$ and $P$ ) allow to rewrite any bi-orthogonal polynomial $p_{m}$ as a linear combination of $p_{j}$ with $n-d_{2} \leq j \leq n$, where $d_{2}+1$ is the degree of the potential entering the weight of orthogonality.

- In section 7, we prove our main result. We compute the mixed correlation function $W_{n}(x, y)$ in terms of polynomials in the window, and in terms of the kernels.

- In section 8, we study some consequences of that formula. In particular, we find new representations of the differential system satisfied by a window, and we compute the spectral curve. We find several new formulae for the spectral curve, and we prove the conjecture of M. Bertola, which claims that the spectral curve is the same curve which was found from loop equations.

- In section 9, we discuss the consequences of that formula in terms of tau functions.

- In section 10, we give some explicit examples of our formulae, namely the gaussian and gaussian elliptical cases.

- Most of the technical proofs are put in the appendix. 


\subsection{Main results}

This article is very technical, and the purpose is to give many formulae for effective computations with bi-orthogonal polynomials. We propose some new formulae in almost every paragraph. Let us mention here the most important ones:

- In section 3.1. we give integral representations of the right $(R)$ and left $(L)$ inverses of $x-Q$. One of the important relations is Eq. (3-30):

$$
L(x)-R(x)=\Psi_{\infty}(x) \hat{\Phi}_{\infty}^{t}(x)
$$

- In section 5, we give determinantal expressions of bi-orthogonal polynomials and their transforms, as well as kernels. For instance the kernel $K_{n}(x, y)$ is:

$$
K_{n+1}(x, y) \propto \operatorname{det}_{n \times n}\left((x-Q)\left(y-P^{t}\right)\right)
$$

- In section [7 we state one of the main results of this paper, i.e. some formulae for the 2-point mixed correlation function $W_{n}(x, y)$ :

$$
\begin{aligned}
W_{n}(x, y)= & 1+\left\langle\operatorname{Tr} \frac{1}{x-M_{1}} \frac{1}{y-M_{2}}\right\rangle \\
= & \operatorname{det}_{n \times n}\left(1+\frac{1}{x-Q} \frac{1}{y-P^{t}}\right) \\
= & \gamma_{n}^{2} K_{n+1}(x, y) J_{n-1}(x, y) \times \\
& \quad \operatorname{det}_{d_{2}+1 \times d_{2}+1}\left(1-\prod_{n}^{n-d_{2}}\left(1-\frac{\vec{\psi}_{n} \vec{\phi}_{n}^{t}}{K_{n+1}}\right) U_{n}\left(1-\frac{\hat{\vec{\psi}}_{n} \hat{\vec{\phi}}_{n}^{t}}{J_{n-1}}\right) \tilde{U}_{n}^{t}\right)
\end{aligned}
$$

and theorem 7.2 gives $W_{n}$ in terms of kernels only.

We also find interesting recursion relations for $W_{n+1}-W_{n}$.

- In section 8, we compute new representations of the differential system $\mathcal{D}_{n}(x)=$ $\Psi_{n}^{\prime}(x) \Psi_{n}(x)^{-1}$, and we compute the spectral curve:

$$
\mathcal{E}_{n}(x, y)=\tilde{t} \operatorname{det}\left(y-\mathcal{D}_{n}(x)\right)=-\gamma_{n}^{2} \operatorname{det}\left(1-U_{n} \tilde{U}_{n}^{t}\right)=\operatorname{det}\left(y-H^{(i j)}(x, x)\right)
$$

The most interesting is that we prove Bertola's conjecture [5]:

$\mathcal{E}_{n}(x, y)=\left(V_{1}^{\prime}(x)+y\right)\left(V_{2}^{\prime}(y)+x\right)-n+\left\langle\operatorname{Tr} \frac{V_{1}^{\prime}(x)-V_{1}^{\prime}\left(M_{1}\right)}{x-M_{1}} \frac{V_{2}^{\prime}(y)-V_{2}^{\prime}\left(M_{2}\right)}{y-M_{2}}\right\rangle$

This conjecture has important consequences in terms of tau functions. Indeed the Miwa-Jimbo-Ueno approach of isomonodromic tau functions [27, 28, 36], generalized in [7, allows to express the tau function in terms of residues of the spectral 
curve, and this formula is particularly convenient for that purpose. It shows that the tau function is the matrix integral, and it shows that some additional parameters could be added to the model.

\section{Definitions and notations about bi-orthogonal polynomials}

This section recalls well known facts about bi-orthogonal polynomials, and stands here just for setting notations and describing known properties. Notations are similar (although with small differences) to those of [10, 11].

\subsection{Measure and integration paths}

Consider the weight:

$$
\omega(x, y)=\mathrm{e}^{-\left(V_{1}(x)+V_{2}(y)+x y\right)},
$$

where $V_{1}$ is a complex polynomial of degree $d_{1}+1$ and $V_{2}$ a complex polynomial of degree $d_{2}+1$ :

$$
V_{1}(x)=\sum_{k=0}^{d_{1}+1} t_{k} x^{k} \quad, \quad V_{2}(y)=\sum_{k=0}^{d_{2}+1} \tilde{t}_{k} y^{k}
$$

We write the leading coefficients of $V_{1}^{\prime}$ and $V_{2}^{\prime}$ :

$$
t=\left(d_{1}+1\right) t_{d_{1}+1} \quad, \quad \tilde{t}=\left(d_{2}+1\right) \tilde{t}_{d_{2}+1} .
$$

We choose a basis of $d_{1}$ contours $\gamma^{(i)}$ with $i=1, \ldots, d_{1}$, going from $\infty$ to $\infty$ in sectors where the integral $\int \mathrm{e}^{-V_{1}(x)} d x$ is convergent, and we choose a basis of $d_{2}$ contours $\tilde{\gamma}^{(i)}$ with $i=1, \ldots, d_{2}$, going from $\infty$ to $\infty$ in sectors where the integral $\int \mathrm{e}^{-V_{2}(y)} d y$ is convergent (see [10, 6]).

Then we choose a dual basis of $d_{1}$ contours $\bar{\gamma}^{(i)}$ with $i=1, \ldots, d_{1}$, going from $\infty$ to $\infty$ in sectors where the integral $\int \mathrm{e}^{+V_{1}(x)} d x$ is convergent, and we choose a dual basis of $d_{2}$ contours $\tilde{\bar{\gamma}}^{(i)}$ with $i=1, \ldots, d_{2}$, going from $\infty$ to $\infty$ in sectors where the integral $\int \mathrm{e}^{+V_{2}(y)} d y$ is convergent, such that:

$$
\gamma^{(i)} \cap \bar{\gamma}^{(j)}=\delta_{i j} \quad, \quad \tilde{\gamma}^{(i)} \cap \tilde{\bar{\gamma}}^{(j)}=\delta_{i j}
$$

Then, we choose $d_{1} d_{2}$ numbers $\kappa_{i, j}$ such that at least one of them is non-vanishing, and we define a path $\Gamma$ :

$$
\Gamma:=\sum_{i=1}^{d_{1}} \sum_{j=1}^{d_{2}} \kappa_{i, j} \gamma^{(i)} \times \tilde{\gamma}^{(j)}
$$


and we define the following measure on $\Gamma$ :

$$
d \mu(x, y)=\mathrm{e}^{-\left(V_{1}(x)+V_{2}(y)+x y\right)} d x d y .
$$

Remark 2.1 Generalized path $\Gamma$ and matrix models

We have introduced the generalized integration contours $\Gamma$, because it is the most general contour on which the measure $d \mu$ can be integrated. It corresponds to a generalization of the hermitean 2-matrix model. Indeed, hermitean matrices have their eigenvalues on the real axis, and the hermitean 2-matrix model Eq. (1-1) corresponds to the case $\Gamma=\mathbf{R} \times \mathbf{R}$.

A generalized path $\Gamma$ can also correspond to a matrix model, with matrices which are not hermitean. It corresponds to normal matrices (i.e. which can be diagonalized by a unitary transformation, but with complex eigenvalues), with pairs of eigenvalues constrained to be on $\Gamma$. This allows to define an ensemble of matrices which is noted $H_{n} \times H_{n}(\Gamma)$, see [18] for more details.

In this normal matrix model, it makes sense to compute matrix expectation values, in particular the mixed correlation function $W_{n}(x, y)=1+\left\langle\operatorname{Tr} \frac{1}{x-M_{1}} \frac{1}{y-M_{2}}\right\rangle$.

\section{More definitions}

We define (see [13, 25]):

$$
h_{0}=\int_{\Gamma} d \mu(x, y)=\sum_{i=1}^{d_{1}} \sum_{j=1}^{d_{2}} \kappa_{i, j} \int_{\gamma^{(i)}} d x \int_{\tilde{\gamma}^{(j)}} d y \mathrm{e}^{-\left(V_{1}(x)+V_{2}(y)+x y\right)} .
$$

And:

$$
\begin{array}{lll}
g^{(i)}(x):=\frac{\sqrt{h_{0}}}{2 i \pi} \int_{\tilde{\bar{\gamma}}^{(i)}} \mathrm{e}^{x y} \mathrm{e}^{V_{2}(y)} d y \quad, i=1, \ldots, d_{2} \quad, \quad g^{(0)}:=0, \\
\tilde{g}^{(i)}(y):=\frac{\sqrt{h_{0}}}{2 i \pi} \int_{\bar{\gamma}^{(i)}} \mathrm{e}^{x y} \mathrm{e}^{V_{1}(x)} d x \quad, i=1, \ldots, d_{1} \quad, \quad \tilde{g}^{(0)}:=0,
\end{array}
$$

which are the independent solutions of the differential equations:

$$
\begin{aligned}
& V_{2}^{\prime}(\partial / \partial x) g^{(i)}(x)=-x g^{(i)}(x) \\
& V_{1}^{\prime}(\partial / \partial y) \tilde{g}^{(i)}(y)=-y \tilde{g}^{(i)}(y) .
\end{aligned}
$$

Then we define the following "concomitents" [13, 25]:

$$
\begin{aligned}
& c^{(i j)}=\frac{1}{2 i \pi} \int_{\gamma^{(i)}} d x \int_{\bar{\gamma}^{(j)}} d x^{\prime} \frac{V_{1}^{\prime}(x)-V_{1}^{\prime}\left(x^{\prime}\right)}{x-x^{\prime}} \mathrm{e}^{V_{1}\left(x^{\prime}\right)-V_{1}(x)} \mathrm{e}^{\left(x^{\prime}-x\right) y}, \\
& \tilde{c}^{(i j)}=\frac{1}{2 i \pi} \int_{\tilde{\gamma}^{(i)}} d y \int_{\tilde{\bar{\gamma}}^{(j)}} d y^{\prime} \frac{V_{2}^{\prime}(y)-V_{2}^{\prime}\left(y^{\prime}\right)}{y-y^{\prime}} \mathrm{e}^{V_{2}\left(y^{\prime}\right)-V_{2}(y)} \mathrm{e}^{\left(y^{\prime}-y\right) x} .
\end{aligned}
$$

The $c^{(i j)}$ 's (resp. $\tilde{c}^{(i j)}$ 's) are independent of $y$ (resp. $x$ ). Due to the dual choice of contours Eq. (2-4), they are normalized:

$$
c^{(i j)}=\delta_{i, j} \quad, \quad \tilde{c}^{(i j)}=\delta_{i, j}
$$


Indeed, integrating by parts, we can replace both $V_{1}^{\prime}(x)$ by $y$ and $V_{1}^{\prime}\left(x^{\prime}\right)$ by $y$. If $i \neq j$, the contours $\gamma^{(i)}$ and $\bar{\gamma}^{(j)}$ do not intersect, and the integration by parts gives no boundary term and the result vanishes. If $i=j$, the two contours intersect and we have a boundary term. A way to compute it, is to write the pole $1 /\left(x-x^{\prime}\right)$ as the sum of a principal part and $2 i \pi \delta\left(x-x^{\prime}\right)$. The principal part is integrated by parts and gives zero as in the $i \neq j$ case, whilst the $\delta$-term corresponds to the boundary term in the integration by parts, and it gives 1 .

This computation was first done by the authors of [13, 25], in the comparison of the two Riemann-Hilbert problems derived for biorthogonal polynomials [10, 32, 29]

\subsection{Bi-orthogonal polynomials}

The monic bi-orthogonal polynomials [33, 34, (if they exist), are uniquely determined by:

$$
p_{n}(x)=x^{n}+\ldots \quad, \quad q_{n}(y)=y^{n}+\ldots
$$

and

$$
\int_{\Gamma} p_{n}(x) q_{m}(y) d \mu(x, y)=h_{n} \delta_{n m}
$$

For given potentials $V_{1}$ and $V_{2}$, bi-orthogonal polynomials exist for almost every choice of $\Gamma$ (in fact they don't exist only for an enumerable set of $\Gamma$ 's, see [10]).

\subsubsection{Wave functions}

We define:

$$
\psi_{n}(x):=\frac{1}{\sqrt{h_{n}}} p_{n}(x) \mathrm{e}^{-V_{1}(x)} \quad, \quad \phi_{n}(y):=\frac{1}{\sqrt{h_{n}}} q_{n}(y) \mathrm{e}^{-V_{2}(y)} .
$$

\subsubsection{Cauchy transforms}

We introduce the Cauchy transforms [9, 10]:

$$
\begin{aligned}
& \hat{\psi}_{n}(y):=\frac{1}{\sqrt{h_{n}}} \mathrm{e}^{V_{2}(y)} \int_{\Gamma} \frac{1}{y-y^{\prime}} p_{n}\left(x^{\prime}\right) d \mu\left(x^{\prime}, y^{\prime}\right), \\
& \hat{\phi}_{n}(x):=\frac{1}{\sqrt{h_{n}}} \mathrm{e}^{V_{1}(x)} \int_{\Gamma} \frac{1}{x-x^{\prime}} q_{n}\left(y^{\prime}\right) d \mu\left(x^{\prime}, y^{\prime}\right) .
\end{aligned}
$$

\subsubsection{Fourier-Laplace transforms}

We also introduce the following functions [16, 17]:

$$
\hat{\psi}_{n}^{(i)}(y):=\int_{\gamma^{(i)}} \psi_{n}(x) \mathrm{e}^{-x y} d x \quad \text { for } i=1, \ldots, d_{1}
$$




$$
\hat{\phi}_{n}^{(i)}(x):=\int_{\tilde{\gamma}^{(i)}} \phi_{n}(y) \mathrm{e}^{-x y} d y \quad \text { for } i=1, \ldots, d_{2} .
$$

They are the Fourier-Laplace transforms of $\psi_{n}$ and $\phi_{n}$. For $i=0$ we will also write:

$$
\hat{\psi}_{n}^{(0)}(y):=\hat{\psi}_{n}(y) \quad, \quad \hat{\phi}_{n}^{(0)}(x):=\hat{\phi}_{n}(x) .
$$

\subsubsection{Third-type functions}

The authors of [13, 25] have introduced the following functions:

$$
\begin{aligned}
& \phi_{n}^{(i)}(y):=\frac{1}{2 i \pi} \frac{1}{\sqrt{h_{n}}} \int_{\bar{\gamma}^{(i)}} d x \int_{\Gamma} d \mu\left(x^{\prime}, y^{\prime}\right) \frac{1}{y-y^{\prime}} \frac{V_{1}^{\prime}(x)-V_{1}^{\prime}\left(x^{\prime}\right)}{x-x^{\prime}} \mathrm{e}^{V_{1}(x)} \mathrm{e}^{x y} q_{n}\left(y^{\prime}\right), \\
& \psi_{n}^{(i)}(x):=\frac{1}{2 i \pi} \frac{1}{\sqrt{h_{n}}} \int_{\tilde{\bar{\gamma}}^{(i)}} d y \int_{\Gamma} d \mu\left(x^{\prime}, y^{\prime}\right) \frac{1}{x-x^{\prime}} \frac{V_{2}^{\prime}(y)-V_{2}^{\prime}\left(y^{\prime}\right)}{y-y^{\prime}} \mathrm{e}^{V_{2}(y)} \mathrm{e}^{x y} p_{n}\left(x^{\prime}\right) .
\end{aligned}
$$

We will also write:

$$
\psi_{n}^{(0)}(x):=\psi_{n}(x) \quad, \quad \phi_{n}^{(0)}(y):=\phi_{n}(y)
$$

Formaly, those functions are the "inverse Fourier transforms" of the $\hat{\psi}$ 's, as described in [10, 29].

\subsection{Semi-infinite vectors and matrices}

We introduce semi-infinite vector notations:

$$
\psi_{\infty}(x)=\left(\psi_{0}(x), \psi_{1}(x), \psi_{2}(x), \ldots\right)^{t} \quad, \quad \phi_{\infty}(y)=\left(\phi_{0}(y), \phi_{1}(y), \phi_{2}(y), \ldots\right)^{t}
$$

and more generaly:

$$
\psi_{\infty}{ }^{(i)}(x)=\left(\psi_{0}^{(i)}(x), \psi_{1}^{(i)}(x), \psi_{2}^{(i)}(x), \ldots\right)^{t}, \phi_{\infty}{ }^{(i)}(y)=\left(\phi_{0}^{(i)}(y), \phi_{1}^{(i)}(y), \phi_{2}^{(i)}(y), \ldots\right)^{t}
$$

And

$$
\hat{\psi_{\infty}}(y)=\left(\hat{\psi_{0}}(y), \hat{\psi}_{1}(y), \hat{\psi}_{2}(y), \ldots\right)^{t} \quad, \quad \hat{\phi_{\infty}}(x)=\left(\hat{\phi}_{0}(x), \hat{\phi}_{1}(x), \hat{\phi}_{2}(x), \ldots\right)^{t}
$$

and more generaly:

$$
{\hat{\psi_{\infty}}}^{(i)}(y)=\left(\hat{\psi}_{0}^{(i)}(y), \hat{\psi}_{1}^{(i)}(y), \hat{\psi}_{2}^{(i)}(y), \ldots\right)^{t},{\hat{\phi_{\infty}}}^{(i)}(x)=\left(\hat{\phi}_{0}^{(i)}(x), \hat{\phi}_{1}^{(i)}(x), \hat{\phi}_{2}^{(i)}(x), \ldots\right)^{t}
$$

We also introduce the basis vectors:

$$
\mathbf{e}_{n}=(\overbrace{0, \ldots, 0}^{n}, 1, \overbrace{0, \ldots}^{\infty})^{t},
$$


i.e. the vector whose only non vanishing component is in the $n+1$ position. It is such that:

$$
\mathbf{e}_{n}^{t} \psi_{\infty}(x)=\psi_{n}(x)
$$

Similarly, we consider the projection matrix:

$$
\Pi_{n}=\operatorname{diag}(\overbrace{1, \ldots, 1}^{n+1}, \overbrace{0, \ldots}^{\infty})=\sum_{j=0}^{n} \mathbf{e}_{j} \mathbf{e}_{j}^{t}
$$

with $n+1$ ones on the diagonal. It is the projector on the span of $\mathbf{e}_{0}, \mathbf{e}_{1}, \ldots, \mathbf{e}_{n}$. We also define:

$$
\Pi^{n}=1-\Pi_{n-1}=\text { projector on the span of } n, n+1, \ldots, \infty
$$

and:

$$
\Pi_{m}^{n}=\sum_{j=n}^{m} \mathbf{e}_{j} \mathbf{e}_{j}^{t}=\Pi^{n} \Pi_{m}=\Pi_{m}-\Pi_{n-1}=\text { projector on the span of } n, \ldots, m .
$$

We also introduce the following $\infty \times\left(d_{2}+1\right)$, (resp. $\left.\infty \times\left(d_{1}+1\right)\right)$ matrices:

$$
\begin{aligned}
\Psi_{\infty} & :=\left(\begin{array}{llll}
\psi_{\infty}{ }^{(0)} & \psi_{\infty}{ }^{(1)} & \ldots & \psi_{\infty}{ }^{\left(d_{2}\right)}
\end{array}\right), \\
\Phi_{\infty} & :=\left(\begin{array}{llll}
\hat{\phi}_{\infty}^{(0)} & \phi_{\infty}{ }^{(1)} & \ldots & \phi_{\infty}^{\left(d_{1}\right)}
\end{array}\right), \\
\hat{\Psi}_{\infty} & :=\left(\begin{array}{llll}
\hat{\psi}_{\infty}^{(0)} & {\hat{\psi_{\infty}}}^{(1)} & \ldots & {\hat{\psi_{\infty}}}^{\left(d_{1}\right)}
\end{array}\right), \\
\hat{\Phi}_{\infty} & :=\left(\begin{array}{llll}
{\hat{\phi_{\infty}}}^{(0)} & {\hat{\phi_{\infty}}}^{(1)} & \ldots & {\hat{\phi_{\infty}}}^{\left(d_{2}\right)}
\end{array}\right) .
\end{aligned}
$$

\subsection{Recursion relations for the bi-orthogonal polynomials}

It is well known ${ }^{3}$ that we have the following recursion relations [15, 9]:

$$
\begin{aligned}
& x \psi_{n}=\sum_{m} Q_{n m} \psi_{m} \quad, \quad y \phi_{n}=\sum_{m} P_{n m} \phi_{m}, \\
& \psi_{n}^{\prime}=\sum_{m} P_{m n} \psi_{m} \quad, \quad \phi_{n}^{\prime}=\sum_{m} Q_{m n} \phi_{m} .
\end{aligned}
$$

Since $V_{1}$ and $V_{2}$ are polynomials, $Q$ and $P$ must be finite-band matrices, i.e.:

$$
\begin{aligned}
& Q_{n m} \neq 0 \quad \text { iff } n-d_{2} \leq m \leq n+1 \\
& P_{n m} \neq 0 \quad \text { iff } n-d_{1} \leq m \leq n+1
\end{aligned}
$$

In vector notations we have:

$$
\begin{array}{cc}
x \psi_{\infty}=Q \psi_{\infty} & , \quad y \phi_{\infty}=P \phi_{\infty} \\
\psi_{\infty}^{\prime}=P^{t} \psi_{\infty} & , \quad \phi_{\infty}^{\prime}=Q^{t} \phi_{\infty}
\end{array}
$$

\footnotetext{
${ }^{3} x$ and $\partial_{x}$ acting on a polynomial, gives a polynomial which can be decomposed on the basis of biorthogonal polynomials.
} 


\subsection{Relations between $\mathrm{Q}$ and $\mathrm{P}$}

The matrices $Q$ and $P$ have the following properties [15, 9, 10]:

$$
\begin{gathered}
{\left[Q, P^{t}\right]=\mathrm{Id} .} \\
Q_{n, n+1}=P_{n, n+1}=\sqrt{\frac{h_{n+1}}{h_{n}}}:=\gamma_{n+1} .
\end{gathered}
$$

$P^{t}+V_{1}^{\prime}(Q)$ is a strictly lower triangular matrix

$$
\left(P^{t}+V_{1}^{\prime}(Q)\right)_{+}=0 \quad, \quad\left(P^{t}+V_{1}^{\prime}(Q)\right)_{n, n-1}=\frac{n}{\gamma_{n}},
$$

and $Q^{t}+V_{2}^{\prime}(P)$ is a strictly lower triangular matrix

$$
\left(Q^{t}+V_{2}^{\prime}(P)\right)_{+}=0 \quad, \quad\left(Q^{t}+V_{2}^{\prime}(P)\right)_{n, n-1}=\frac{n}{\gamma_{n}} .
$$

\subsection{Recursion relations for the Cauchy transforms}

The Cauchy transforms also satisfy recursion relations [10]:

$$
\begin{gathered}
y \hat{\psi_{\infty}}(y)=P^{t} \hat{\psi_{\infty}}(y)+\frac{1}{\phi_{0}(y)} \mathbf{e}_{0} \quad, \quad x \hat{\phi_{\infty}}(x)=Q^{t} \hat{\phi_{\infty}}(x)+\frac{1}{\psi_{0}(x)} \mathbf{e}_{0}, \\
\hat{\psi}_{\infty}^{\prime}=-Q \hat{\psi_{\infty}}+\frac{1}{\phi_{0}} \frac{V_{2}^{\prime}(y)-V_{2}^{\prime}\left(P^{t}\right)}{y-P^{t}} \mathbf{e}_{0} \\
\hat{\phi}_{\infty}^{\prime}=-P \hat{\phi_{\infty}}+\frac{1}{\psi_{0}} \frac{V_{1}^{\prime}(x)-V_{1}^{\prime}\left(Q^{t}\right)}{x-Q^{t}} \mathbf{e}_{0}
\end{gathered}
$$

\subsection{Recursion relations for the Fourier-Laplace transforms}

For $i \neq 0$ we have 9, 10]:

$$
\begin{array}{cc}
y{\hat{\psi_{\infty}}}^{(i)}=P^{t}{\hat{\psi_{\infty}}}^{(i)} \quad, \quad x{\hat{\phi_{\infty}}}^{(i)}=Q^{t}{\hat{\phi_{\infty}}}^{(i)}, \\
{\hat{\psi_{\infty}}}^{(i)}=-Q{\hat{\psi_{\infty}}}^{(i)} \quad, \quad{\hat{\phi_{\infty}}}^{(i)}=-P{\hat{\phi_{\infty}}}^{(i)} .
\end{array}
$$

Notice that they satisfy the same recursion relation as the Cauchy transforms without the non-homogeneous term. 


\subsection{Recursion relations for the third type functions}

For $i \neq 0$ we have [10, 13, 25]:

$$
\begin{gathered}
x \psi_{\infty}^{(i)}=Q \psi_{\infty}{ }^{(i)}+\left(\frac{V_{2}^{\prime}(\partial / \partial x)-V_{2}^{\prime}\left(P^{t}\right)}{\partial / \partial x-P^{t}}\right) \mathbf{e}_{0} g^{(i)} \\
=Q \psi_{\infty}{ }^{(i)}+\frac{\sqrt{h_{0}}}{2 i \pi} \int_{\tilde{\gamma}^{(i)}} d y \mathrm{e}^{V_{2}(y)} \mathrm{e}^{x y}\left(\frac{V_{2}^{\prime}(y)-V_{2}^{\prime}\left(P^{t}\right)}{y-P^{t}}\right) \mathbf{e}_{0} \\
\psi_{\infty}^{\prime(i)}=P^{t} \psi_{\infty}^{(i)}-g^{(i)} \mathbf{e}_{0} \\
y \phi_{\infty}^{(i)}=P \phi_{\infty}^{(i)}+\left(\frac{V_{1}^{\prime}(\partial / \partial y)-V_{1}^{\prime}\left(Q^{t}\right)}{\partial / \partial y-Q^{t}}\right) \mathbf{e}_{0} \tilde{g}^{(i)} \\
\phi_{\infty}^{\prime(i)}=Q^{t} \phi_{\infty}^{(i)}-\tilde{g}^{(i)} \mathbf{e}_{0} .
\end{gathered}
$$

Notice that they satisfy the same recursion relation as the wave functions, with an additional non-homogeneous term.

\section{Inverses}

The formula for mixed correlation functions found in [8], is written in terms of the inverse operators of $x-Q$ and $y-P$, thus we study them in detail in this section. $x-Q$ and $y-P$ also have distinct right and left inverses, which were shown to play a crucial role in the notion of folding onto a window in [11].

\subsection{Inverse}

By definition, the infinite matrix $1 /(x-Q)$ has elements:

$$
\begin{aligned}
\left(\frac{1}{x-Q}\right)_{n m} & =\frac{1}{\sqrt{h_{n} h_{m}}} \int_{\Gamma} q_{m}\left(y^{\prime}\right) \frac{1}{x-x^{\prime}} p_{n}\left(x^{\prime}\right) d \mu\left(x^{\prime}, y^{\prime}\right) \\
& =\psi_{n}(x) \hat{\phi}_{m}(x)+R_{n m}(x),
\end{aligned}
$$

where

$$
R_{n m}(x):=\frac{-1}{\sqrt{h_{n} h_{m}}} \int_{\Gamma} q_{m}\left(y^{\prime}\right) \frac{p_{n}(x)-p_{n}\left(x^{\prime}\right)}{x-x^{\prime}} d \mu\left(x^{\prime}, y^{\prime}\right)
$$

which is a polynomial in $x$ of degree $n-1$.

In vector notations we have:

$$
\frac{1}{x-Q}=\psi_{\infty}(x){\hat{\phi_{\infty}}}^{t}(x)+R(x) .
$$


Similarly

$$
\frac{1}{y-P}=\phi_{\infty}(y){\hat{\psi_{\infty}}}^{t}(y)+\tilde{R}(y)
$$

where

$$
\tilde{R}_{m n}(y):=-\frac{1}{\sqrt{h_{n} h_{m}}} \int_{\Gamma} \frac{q_{n}(y)-q_{n}\left(y^{\prime}\right)}{y-y^{\prime}} p_{m}\left(x^{\prime}\right) d \mu\left(x^{\prime}, y^{\prime}\right) .
$$

\subsection{Right inverse}

The semi-infinite matrix $R(x)$ (resp. $\tilde{R}(y)$ ) is polynomial in $x$ (resp. $y$ ) and is strictly lower triangular:

$$
\left.R_{n m}=0 \quad \text { if } m \geq n \quad \text { (resp. } \tilde{R}_{n m}=0 \quad \text { if } m \geq n\right) .
$$

It is a right inverse [1] of $(x-Q)(\operatorname{resp} .(y-P))$ :

$$
(x-Q) R(x)=\mathrm{Id} \quad(\operatorname{resp} .(y-P) \tilde{R}(y)=\mathrm{Id}) .
$$

But it is not a left inverse, we have:

$$
R(x)(x-Q)=\operatorname{Id}-\frac{\psi_{\infty}(x) \mathbf{e}_{0}^{t}}{\psi_{0}(x)} \quad\left(\operatorname{resp} . \tilde{R}(y)(y-P)=\operatorname{Id}-\frac{\phi_{\infty}(y) \mathbf{e}_{0}^{t}}{\phi_{0}(y)}\right) .
$$

Notice that

Notice that we have:

$$
R_{n, n-1}=-\frac{1}{\gamma_{n}}=\tilde{R}_{n, n-1}
$$

$$
\begin{gathered}
\frac{\psi_{n}(x)-\psi_{n}\left(x^{\prime}\right)}{x-x^{\prime}}=-\sum_{m} R_{n m}(x) \psi_{m}\left(x^{\prime}\right) \\
\text { resp. } \left.\frac{\phi_{n}(y)-\phi_{n}\left(y^{\prime}\right)}{y-y^{\prime}}=-\sum_{m} \tilde{R}_{n m}(y) \phi_{m}\left(y^{\prime}\right)\right) .
\end{gathered}
$$

In particular at $x=x^{\prime}$ :

$$
\begin{aligned}
& \psi_{\infty}^{\prime}(x)=-R(x) \psi_{\infty}(x)=P^{t} \psi_{\infty}(x) \\
& \left(\operatorname{resp} . \phi_{\infty}^{\prime}(y)=-\tilde{R}(y) \phi_{\infty}(y)=Q^{t} \phi_{\infty}(y)\right) .
\end{aligned}
$$

Notice also that:

$$
R\left(x_{1}\right) R\left(x_{2}\right)=-\frac{R\left(x_{1}\right)-R\left(x_{2}\right)}{x_{1}-x_{2}}
$$

and

$$
\left[R\left(x_{1}\right), R\left(x_{2}\right)\right]=0 .
$$

It can be found, by solving directely the system $(x-Q) R(x)=1$ for a lower triangular matrix $R$, that:

$$
R_{n m}(x)= \begin{cases}\frac{-1}{\gamma_{m+1} \ldots \gamma_{n}} \operatorname{det}\left(\Pi_{m+1}^{n-1}(x-Q) \Pi_{m+1}^{n-1}\right) & \text { for } m \leq n-2 \\ \frac{-1}{\gamma_{n}} & \text { for } m=n-1 \\ 0 & \text { for } m \geq n\end{cases}
$$




\subsection{Left inverse}

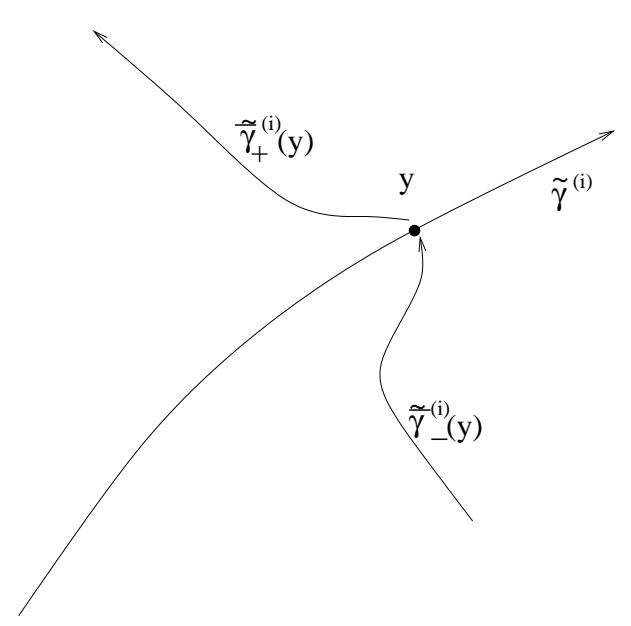

Figure 1: Definition of the contours $\tilde{\bar{\gamma}}_{+}^{(i)}(y)$ and $\tilde{\bar{\gamma}}_{-}^{(i)}(y)$.

Consider $y \in \tilde{\gamma}^{(i)}$, then deform the contour $\tilde{\bar{\gamma}}^{(i)}$ so that it crosses $\tilde{\gamma}^{(i)}$ at $y$, and define $\tilde{\bar{\gamma}}_{-}^{(i)}(y)$ the part of $\tilde{\bar{\gamma}}^{(i)}$ which stands on the right of $\tilde{\gamma}^{(i)}$ and which ends at $y$, and $\tilde{\bar{\gamma}}_{+}^{(i)}(y)$ the part of $\tilde{\bar{\gamma}}^{(i)}$ which stands on the left of $\tilde{\gamma}^{(i)}$ and which starts at $y$ (see fig (1). Then define:

$$
L_{n m}(x):=\frac{-1}{2 i \pi} \sum_{i=1}^{d_{2}} \int_{\tilde{\gamma}^{(i)}} d y \phi_{m}(y) \mathrm{e}^{-x y}\left(\int_{\tilde{\bar{\gamma}}_{+}^{(j)}(y)} d y^{\prime \prime}+\int_{\tilde{\bar{\gamma}}_{-}^{(j)}(y)} d y^{\prime \prime}\right) \hat{\psi}_{n}\left(y^{\prime \prime}\right) \mathrm{e}^{x y^{\prime \prime}}
$$

It has the following properties:

Theorem $3.1 L_{n m}(x)$ is a polynomial in $x$.

$L$ is upper triangular, with $L_{n m}(x)=0$ if $n+d_{2}>m$.

$L$ is a left inverse of $x-Q$ :

$$
L(x)(x-Q)=1
$$

We have:

$$
L_{n m}(x)=\left(\frac{1}{x-Q}\right)_{n m}+\sum_{i=1}^{d_{2}} \psi_{n}^{(i)}(x) \hat{\phi}_{m}^{(i)}(x)
$$

proof:

The proof is in appendix $\mathrm{A}$ 


\section{Alternative definition 1}

It can be found, by solving directely the system $L(x)(x-Q)=1$ for an upper triangular matrix $L$, that:

$$
L_{n m}(x)= \begin{cases}\frac{-1}{Q_{n+d_{2}, n} \cdots Q_{m, m-d_{2}}} \operatorname{det}\left(\Pi_{n+d_{2}}^{m-1}(x-Q) \Pi_{n+1}^{m-d_{2}}\right) & \text { for } m>n+d_{2} \\ \frac{-1}{Q_{n+d_{2}, n}} & \text { for } m=n+d_{2} \\ 0 & \text { for } m<n+d_{2}\end{cases}
$$

This proves that there is only one left inverse of $x-Q$ which is upper triangular.

\section{Alternative definition 2}

Define:

$$
l(x):=\sum_{i} \frac{\tilde{R}^{t}\left(y_{i}(x)\right)}{V_{2}^{\prime \prime}\left(y_{i}(x)\right)}
$$

where $y_{i}(x)$ are the $d_{2}$ solutions of $V_{2}^{\prime}(y)+x=0$. Notice that $l(x)$ is an upper triangular matrix such that:

$$
l_{n m}(x)=0 \quad \text { if } n+d_{2}>m .
$$

The left inverse [1] of $x-Q$ is given by:

$$
\begin{aligned}
& L(x) \quad:=\quad-l(x) \frac{1}{1+\left(V_{2}^{\prime}\left(P^{t}\right)+Q\right) l(x)} \\
& =\quad-l(x)+l(x)\left(V_{2}^{\prime}\left(P^{t}\right)+Q\right) l(x) \\
& -l(x)\left(V_{2}^{\prime}\left(P^{t}\right)+Q\right) l(x)\left(V_{2}^{\prime}\left(P^{t}\right)+Q\right) l(x)+\ldots .
\end{aligned}
$$

Since $l(x)$ and $V_{2}^{\prime}\left(P^{t}\right)+Q$ are strictly upper triangular matrices, each entry of that infinite sum, is actualy a finite sum. $L(x)$ is a strictly upper triangular matrix, such that:

$$
L_{n m}(x)=0 \quad \text { if } n+d_{2}>m .
$$

This definition gives clearly a left inverse of $x-Q$, and since the upper triangular left inverse of $x-Q$ is unique, it must coincide with the first definition.

Remark: $l$ computes the Euclidean division of $q_{m}$ by $V_{2}^{\prime}(y)+x$ :

$$
\phi_{m}(y)=-\left(V_{2}^{\prime}(y)+x\right) \sum_{n} l_{n m}(x) \phi_{n}(y)+\left(\operatorname{deg} \leq d_{2}-1\right) .
$$

Similarly, we define:

$$
\tilde{l}(y):=\sum_{i} \frac{R^{t}\left(x_{i}(y)\right)}{V_{1}^{\prime \prime}\left(x_{i}(y)\right)}
$$


and the left inverse of $y-P$ is:

$$
\begin{array}{rlrl}
\tilde{L}(y) \quad:= & -\tilde{l}(y) & \frac{1}{1+\left(V_{1}^{\prime}\left(Q^{t}\right)+P\right) \tilde{l}(y)} \\
& = & -\tilde{l}(y) & +\tilde{l}(y)\left(V_{1}^{\prime}\left(Q^{t}\right)+P\right) \tilde{l}(y) \\
& & & -\tilde{l}(y)\left(V_{1}^{\prime}\left(Q^{t}\right)+P\right) \tilde{l}(y)\left(V_{1}^{\prime}\left(Q^{t}\right)+P\right) \tilde{l}(y)+\ldots .
\end{array}
$$

$(3-27)$

\subsection{Relationship between right and left inverses}

We have:

$$
\begin{aligned}
\left(\frac{1}{x-Q}\right)_{n m} & =R_{n m}(x)+\psi_{n}^{(0)}(x) \hat{\phi}_{m}^{(0)}(x) \\
& =L_{n m}(x)-\sum_{i=1}^{d_{2}} \psi_{n}^{(i)}(x) \hat{\phi}_{m}^{(i)}(x)
\end{aligned}
$$

i.e. in vector notations

$$
\frac{1}{x-Q}=L(x)-\sum_{i=1}^{d_{2}} \psi_{\infty}{ }^{(i)}(x){\hat{\phi_{\infty}}}^{(i) t}(x)=R(x)+\psi_{\infty}(x){\hat{\phi_{\infty}}}^{t}(x) .
$$

This implies:

$$
L(x)-R(x)=\sum_{i=0}^{d_{2}} \psi_{\infty}{ }^{(i)}(x){\hat{\phi_{\infty}}}^{(i) t}(x)=\Psi_{\infty} \hat{\Phi}_{\infty}^{t}
$$

which is a matrix of rank $d_{2}+1$.

Since $R$ is lower triangular, we have, for $n \leq m$ :

$$
L_{n m}(x)=\sum_{i=0}^{d_{2}} \psi_{n}^{(i)}(x) \hat{\phi}_{m}^{(i)}(x) \quad \text { if } n \leq m,
$$

and since $L$ is upper triangular, we have for $n \geq m-d_{2}+1$ :

$$
R_{n m}(x)=-\sum_{i=0}^{d_{2}} \psi_{n}^{(i)}(x) \hat{\phi}_{m}^{(i)}(x) \quad \text { if } n \geq m-d_{2}+1 .
$$

Notice that if $n \leq m \leq n+d_{2}-1$, we have:

$$
\sum_{i=0}^{d_{2}} \psi_{n}^{(i)}(x) \hat{\phi}_{m}^{(i)}(x)=0 \quad \text { if } n \leq m \leq n+d_{2}-1
$$

and thus:

$$
\left(\frac{1}{x-Q}\right)_{n m}= \begin{cases}\psi_{n}(x) \hat{\phi}_{m}(x) & \text { if } n \leq m \\ -\sum_{i=1}^{d_{2}} \psi_{n}^{(i)}(x) \hat{\phi}_{m}^{(i)}(x) & \text { if } n \geq m-d_{2}+1\end{cases}
$$




\section{Kernels}

The kernels are the building blocks of non-mixed expectation values [16, 2, and we need them because they will appear in mixed expectation values as well. Thus we recall their definitions and some properties below.

\subsection{Definition of the kernels}

\subsection{1 $K$ kernels}

We introduce the kernel:

$$
K_{n}(x, y):=\sum_{m=0}^{n-1} \phi_{m}(y) \psi_{m}(x)=\phi_{\infty}^{t}(y) \Pi_{n-1} \psi_{\infty}(x)
$$

and more generaly:

$$
\begin{aligned}
K_{n}^{(i, j)}(x, y) & :=\sum_{m=0}^{n-1} \phi_{m}^{(i)}(y) \psi_{m}^{(j)}(x)+K_{0}^{(i, j)}(x, y) \\
& =\phi_{\infty}{ }^{(i) t}(y) \Pi_{n-1} \psi_{\infty}{ }^{(j)}(x)+K_{0}^{(i, j)}(x, y)
\end{aligned}
$$

where

$$
\begin{aligned}
K_{0}^{(0,0)}:=0 & K_{0}^{(i, 0)}(x, y):=-\frac{1}{2 i \pi} \mathrm{e}^{-V_{1}(x)} \int_{\bar{\gamma}^{(i)}} \frac{1}{x-x^{\prime}} \mathrm{e}^{V_{1}\left(x^{\prime}\right)} \mathrm{e}^{x^{\prime} y} d x^{\prime} \\
& K_{0}^{(0, j)}(x, y):=-\frac{1}{2 i \pi} \mathrm{e}^{-V_{2}(y)} \int_{\tilde{\bar{\gamma}}^{(j)}} \frac{1}{y-y^{\prime}} \mathrm{e}^{V_{2}\left(y^{\prime}\right)} \mathrm{e}^{x y^{\prime}} d y^{\prime} \\
K_{0}^{(i, j)}(x, y):= & \frac{1}{(2 i \pi)^{2}} \int_{\tilde{\bar{\gamma}}^{(i)}} d x^{\prime \prime} \int_{\bar{\gamma}^{(j)}} d y^{\prime \prime} \int_{\Gamma} d \mu\left(x^{\prime}, y^{\prime}\right) \mathrm{e}^{V_{1}\left(x^{\prime \prime}\right)} \mathrm{e}^{V_{2}\left(y^{\prime \prime}\right)} \mathrm{e}^{x y^{\prime \prime}+x^{\prime \prime} y} \\
& \left(\frac{1}{\left(x-x^{\prime}\right)\left(x-x^{\prime \prime}\right)} \frac{1}{V_{2}^{\prime}\left(y^{\prime}\right)-V_{2}^{\prime}\left(y^{\prime \prime}\right)}\right. \\
y^{\prime}-y^{\prime \prime} & \left.\frac{1}{\left(y-y^{\prime}\right)\left(y-y^{\prime \prime}\right)} \frac{V_{1}^{\prime}\left(x^{\prime}\right)-V_{1}^{\prime}\left(x^{\prime \prime}\right)}{x^{\prime}-x^{\prime \prime}}\right)
\end{aligned}
$$

\subsubsection{J kernels}

We also introduce the kernels:

$$
J_{n}(x, y):=J_{0}(x, y)-\sum_{m=0}^{n-1} \hat{\phi}_{m}(x) \hat{\psi}_{m}(y):=J_{0}(x, y)-\hat{\phi_{\infty}}(x)^{t} \Pi_{n-1} \hat{\psi_{\infty}}(y)
$$

and more generaly

$$
J_{n}^{(i, j)}(x, y):=J_{0}^{(i, j)}(x, y)-\sum_{m=0}^{n-1} \hat{\phi}_{m}^{(i)}(x) \hat{\psi}_{m}^{(j)}(y)
$$


where

$$
\begin{gathered}
J_{0}(x, y):=J_{0}^{(0,0)}(x, y):=\mathrm{e}^{V_{1}(x)} \mathrm{e}^{V_{2}(y)} \int_{\Gamma} \frac{1}{x-x^{\prime}} \frac{1}{y-y^{\prime}} d \mu\left(x^{\prime}, y^{\prime}\right) \\
J_{0}^{(0, i)}(x, y):=\mathrm{e}^{V_{1}(x)} \int_{\gamma^{(i)}} \frac{1}{x-x^{\prime}} \mathrm{e}^{-V_{1}\left(x^{\prime}\right)} \mathrm{e}^{-x^{\prime} y} d x^{\prime} \\
J_{0}^{(i, 0)}(x, y):=\mathrm{e}^{V_{2}(y)} \int_{\tilde{\gamma}^{(i)}} \frac{1}{y-y^{\prime}} \mathrm{e}^{-V_{2}\left(y^{\prime}\right)} \mathrm{e}^{-x y^{\prime}} d y^{\prime} \\
J_{0}^{(i, j)}(x, y):=\mathrm{e}^{-x y}
\end{gathered}
$$

Sometimes one writes abusively: $J_{n}(x, y)=\sum_{m=n}^{\infty} \hat{\phi}_{m}(x) \hat{\psi}_{m}(y)={\hat{\phi_{\infty}}}^{t} \Pi^{n} \hat{\psi_{\infty}}$.

\subsection{3 $H$ kernels}

We also introduce the kernels:

$$
H_{n}^{(i j)}\left(x_{1}, x_{2}\right):=\sum_{m=0}^{n-1} \hat{\phi}_{m}^{(i)}\left(x_{1}\right) \psi_{m}^{(j)}\left(x_{2}\right)+H_{0}^{(i j)}\left(x_{1}, x_{2}\right)
$$

where

$$
\begin{gathered}
H_{0}^{(i, j)}\left(x_{1}, x_{2}\right):=\frac{1}{x_{2}-x_{1}} \frac{\mathrm{e}^{V_{1}\left(x_{1}\right)-V_{1}\left(x_{2}\right)}}{2 i \pi} \int_{\tilde{\bar{\gamma}}^{(j)}} d y \int_{\tilde{\gamma}^{(i)}} d y^{\prime} \frac{V_{2}^{\prime}(y)-V_{2}^{\prime}\left(y^{\prime}\right)}{y-y^{\prime}} \frac{\omega\left(x_{1}, y^{\prime}\right)}{\omega\left(x_{2}, y\right)} \\
H_{0}^{(0, j)}\left(x_{1}, x_{2}\right):=\frac{\mathrm{e}^{V_{1}\left(x_{1}\right)-V_{1}\left(x_{2}\right)}}{2 i \pi} \int_{\tilde{\bar{\gamma}}^{(j)}} d y \int_{\Gamma} d x^{\prime} d y^{\prime} \frac{V_{2}^{\prime}(y)-V_{2}^{\prime}\left(y^{\prime}\right)}{y-y^{\prime}} \frac{1}{x^{\prime}-x_{1}} \frac{1}{x^{\prime}-x_{2}} \frac{\omega\left(x^{\prime}, y^{\prime}\right)}{\omega\left(x_{2}, y\right)} \\
H_{0}^{(i, 0)}\left(x_{1}, x_{2}\right):=0 \\
H_{0}^{(0,0)}\left(x_{1}, x_{2}\right):=\frac{1}{x_{2}-x_{1}} \mathrm{e}^{V_{1}\left(x_{1}\right)-V_{1}\left(x_{2}\right)}
\end{gathered}
$$

Notice that at $x_{1}=x_{2}$ we have:

$$
\lim _{x_{1} \rightarrow x_{2}}\left(x_{2}-x_{1}\right) H_{n}^{(i, j)}\left(x_{1}, x_{2}\right)=\delta_{i, j}
$$

\subsection{4 $\tilde{H}$ kernels}

Similarly:

$$
\tilde{H}_{n}^{(i j)}\left(y, y^{\prime}\right):=\sum_{m=0}^{n-1} q_{m}^{(i)}(y) \hat{p}_{m}^{(j)}\left(y^{\prime}\right)+\tilde{H}_{0}^{(i j)}\left(y, y^{\prime}\right)
$$




\subsection{Christoffel-Darboux matrices}

We define the Christoffel-Darboux matrices [9]:

$$
\begin{aligned}
& A_{n}:=\left[\Pi_{n-1}, Q\right]=\gamma_{n} \mathbf{e}_{n-1} \mathbf{e}_{n}^{t}-\left(1-\Pi_{n-1}\right) Q \Pi_{n-1}, \\
& B_{n}:=\left[\Pi_{n-1}, P\right]=\gamma_{n} \mathbf{e}_{n-1} \mathbf{e}_{n}^{t}-\left(1-\Pi_{n-1}\right) P \Pi_{n-1} .
\end{aligned}
$$

Notice that since $Q$ (resp. $P$ ) is finite band, $A_{n}$ (resp. $B_{n}$ ) is non-vanishing only in a sub-block of size $d_{2}+1 \times d_{2}+1$ (resp. $d_{1}+1 \times d_{1}+1$ ), i.e.

$$
\begin{aligned}
& \left(A_{n}\right)_{i j} \neq 0 \text { iff } n-d_{2} \leq i \leq n \text { and } n-1 \leq j \leq n+d_{2}-1 \\
& \left(B_{n}\right)_{i j} \neq 0 \text { iff } n-d_{1} \leq i \leq n \text { and } n-1 \leq j \leq n+d_{1}-1
\end{aligned}
$$

We say that $A_{n}$ and $B_{n}$ are "small matrices", i.e. their size is not growing with $n$.

\subsection{Christoffel-Darboux theorems}

Using the recursion relations Eq. (2-39) to Eq. (2-56), we have the following ChristoffelDarboux theorems [9, 10]:

\section{Theorem 4.1}

$$
\begin{aligned}
\left(x_{2}-x_{1}\right) H_{n}^{(i j)}\left(x_{1}, x_{2}\right) & ={\hat{\phi_{\infty}}}^{(i) t}\left(x_{1}\right) A_{n} \psi_{\infty}{ }^{(j)}\left(x_{2}\right) \\
\left(\partial_{x_{1}}+\partial_{x_{2}}\right) H_{n}^{(i j)}\left(x_{1}, x_{2}\right) & =-{\hat{\phi_{\infty}}}^{(i) t}\left(x_{1}\right) B_{n}^{t} \psi_{\infty}{ }^{(j)}\left(x_{2}\right) \\
(x-\partial / \partial y) K_{n}^{(i j)}(x, y) & =\phi_{\infty}{ }^{(i) t}(y) A_{n} \psi_{\infty}{ }^{(j)}(x) \\
(y-\partial / \partial x) K_{n}^{(i j)}(x, y) & =\phi_{\infty}^{(i) t}(y) B_{n}^{t} \psi_{\infty}^{(j)}(x) \\
(x+\partial / \partial y) J_{n}(x, y) & ={\hat{\phi_{\infty}}}^{(i) t}(y) \hat{A}_{n}{\hat{\psi_{\infty}}}^{(j)}(x) \\
(y+\partial / \partial x) J_{n}(x, y) & ={\hat{\phi_{\infty}}}^{(i) t}(y) B_{n}^{t}{\hat{\psi_{\infty}}}^{(j)}(x)
\end{aligned}
$$

In particular, at $x_{1}=x_{2}=x$ in Eq. (4-24), and using Eq. (4-18), we recover the duality of $[9]$ :

$$
{\hat{\phi_{\infty}}}^{(i) t}(x) A_{n} \psi_{\infty}{ }^{(j)}(x)=\delta_{i, j}
$$

and we find:

$$
H_{n}^{(i j)}(x, x)={\hat{\phi_{\infty}}}^{(i) t}(x) A_{n} \psi_{\infty}^{\prime(j)}(x)
$$




\section{Determinantal formula for the orthogonal poly- nomials and kernels}

\subsection{Determinantal formulae}

The following formulae are very useful to express kernels or polynomials, as well as the Cauchy transforms, as $n \times n$ determinants. The proofs are given in appendix B.

1. - determinant of $\Pi_{n-1}(x-Q) \Pi_{n-1}$ :

$$
\operatorname{det}\left(\Pi_{n-1}(x-Q) \Pi_{n-1}\right)=p_{n}(x) \quad, \quad \operatorname{det}\left(\Pi_{n-1}(y-P) \Pi_{n-1}\right)=q_{n}(y)
$$

2. - determinant of $\Pi_{n-1} \frac{1}{x-Q} \Pi_{n-1}$ :

$$
\begin{aligned}
& \operatorname{det}\left(\Pi_{n-1} \frac{1}{x-Q} \Pi_{n-1}\right)=\frac{\mathrm{e}^{-V_{1}(x)}}{\sqrt{h_{n-1}}} \hat{\phi}_{n-1}(x) \\
& \operatorname{det}\left(\Pi_{n-1} \frac{1}{y-P} \Pi_{n-1}\right)=\frac{\mathrm{e}^{-V_{2}(y)}}{\sqrt{h_{n-1}}} \hat{\psi}_{n-1}(y)
\end{aligned}
$$

3. kernel $K_{n}$ :

$$
\operatorname{det}\left(\Pi_{n-1}(x-Q)\left(y-P^{t}\right) \Pi_{n-1}\right)=h_{n} \mathrm{e}^{V_{1}(x)+V_{2}(y)} K_{n+1}(x, y)
$$

4. • kernel $J_{n}$ :

$$
\operatorname{det}\left(\Pi_{n-1} \frac{1}{x-Q} \frac{1}{y-P^{t}} \Pi_{n-1}\right)=\frac{\mathrm{e}^{-V_{1}(x)-V_{2}(y)}}{h_{n-1}} J_{n-1}(x, y)
$$

\subsection{Inverses}

It is usefull to compute also the inverses (i.e. all minors) of the previous matrices. The proofs are given in appendix 11. The following formula give inverses of $n \times n$ matrices.

1. - inverse of $\Pi_{n-1}(x-Q) \Pi_{n-1}$ :

$$
\left(\Pi_{n-1}(x-Q) \Pi_{n-1}\right)^{-1}=\Pi_{n-1}\left(1-\frac{\psi_{\infty} \mathbf{e}_{n}^{t}}{\psi_{n}}\right) \Pi_{n} R \Pi_{n-1}
$$

2. • inverse of $\prod_{n-1} \frac{1}{x-Q} \Pi_{n-1}$ :

$$
\left(\Pi_{n-1} \frac{1}{x-Q} \Pi_{n-1}\right)^{-1}=\Pi_{n-1}\left(1+\frac{\mathbf{e}_{n-1}{\hat{\phi_{\infty}}}^{t}}{\hat{\phi}_{n-1}} \Pi^{n}\right)(x-Q) \Pi_{n-1}
$$


3. • inverse of $\Pi_{n-1}(x-Q)\left(y-P^{t}\right) \Pi_{n-1}$ :

$$
\left(\Pi_{n-1}(x-Q)\left(y-P^{t}\right) \Pi_{n-1}\right)^{-1}=\Pi_{n-1} \tilde{R}^{t} \Pi_{n}\left(1-\frac{1}{K_{n+1}} \psi_{\infty} \phi_{\infty}^{t}\right) \Pi_{n} R \Pi_{n-1}
$$

4. • inverse of $\Pi_{n-1} \frac{1}{x-Q} \frac{1}{y-P^{t}} \Pi_{n-1}$ :

$$
\begin{aligned}
& \left(\Pi_{n-1} \frac{1}{x-Q} \frac{1}{y-P^{t}} \Pi_{n-1}\right)^{-1} \\
= & \Pi_{n-1}\left(y-P^{t}\right)\left(\Pi_{n-2}+\frac{1}{J_{n-1}}\left(1-\Pi_{n-2}\right){\hat{\psi_{\infty}}}^{\hat{\phi}_{\infty}^{t}}\left(1-\Pi_{n-2}\right)\right)(x-Q) \Pi_{n-1}
\end{aligned}
$$

5. • equivalent formula for $\prod_{n-1} \frac{1}{x-Q} \frac{1}{y-P^{t}} \Pi_{n-1}$ :

$$
\begin{aligned}
& \Pi_{n-1} \frac{1}{x-Q} \frac{1}{y-P^{t}} \Pi_{n-1} \\
= & \Pi_{n-1} \frac{1}{x-Q} \Pi_{n-1} \frac{1}{y-P^{t}} \Pi_{n-1}+J_{n} \Pi_{n-1} \psi_{\infty} \phi_{\infty}{ }^{t} \Pi_{n-1} \\
= & \Pi_{n-1} \frac{1}{x-Q} \Pi_{n-2} \frac{1}{y-P^{t}} \Pi_{n-1}+J_{n-1} \Pi_{n-1} \psi_{\infty} \phi_{\infty}{ }^{t} \Pi_{n-1}
\end{aligned}
$$

$(5-10)$

\section{Windows}

The Christoffel Darboux theorems of section 4.3, show that the kernels can be computed in terms of only $\psi_{m}$ with $n-d_{2} \leq m \leq n$ and $\hat{\phi}_{m}$ with $n-1 \leq m \leq n+d_{2}-1$ (resp. $\phi_{m}$ with $n-d_{1} \leq m \leq n$ and $\hat{\psi}_{m}$ with $\left.n-1 \leq m \leq n+d_{1}-1\right)$. We thus introduce the following vectors, called "windows" [9, 10, 11]:

$$
\vec{\psi}_{n}(x)=\left(\psi_{n-d_{2}}, \psi_{n-d_{2}+1}, \ldots, \psi_{n-1}, \psi_{n}\right)^{t}=\Pi_{n}^{n-d_{2}} \psi_{\infty}
$$

and similarly:

$$
\vec{\phi}_{n}(y)=\Pi_{n}^{n-d_{1}} \phi_{\infty} \quad, \quad \hat{\vec{\psi}}_{n}(y)=\Pi_{n+d_{1}-1}^{n-1} \hat{\psi_{\infty}} \quad, \quad \hat{\vec{\phi}}_{n}(y)=\Pi_{n+d_{2}-1}^{n-1} \hat{\phi_{\infty}}
$$

We also introduce the following matrices, called "windows" 9, 10, 11]:

$$
\begin{array}{ll}
\Psi_{n}(x)_{m i}:=\psi_{m}^{(i)}(x) \quad, \quad m=n-d_{2}, \ldots, n \quad, & i=0, \ldots, d_{2} \\
\Phi_{n}(y)_{m i}:=\phi_{m}^{(i)}(y) \quad, \quad m=n-d_{1}, \ldots, n \quad, \quad i=0, \ldots, d_{1}
\end{array}
$$




$$
\begin{aligned}
& \hat{\Psi}_{n}(y)_{m i}:=\hat{\psi}_{m}^{(i)}(y) \quad, \quad m=n-1, \ldots, n+d_{1}-1 \quad, \quad i=0, \ldots, d_{1} \\
& \hat{\Phi}_{n}(x)_{m i}:=\hat{\phi}_{m}^{(i)}(x) \quad, \quad m=n-1, \ldots, n+d_{2}-1 \quad, \quad i=0, \ldots, d_{2}
\end{aligned}
$$

The relationship Eq. (4-30) implies the duality (found in [9]):

$$
\hat{\Phi}_{n}^{t}(x) A_{n} \Psi_{n}(x)=\operatorname{Id}_{d_{2}+1}
$$

and similarly:

$$
\Phi_{n}^{t}(y) B_{n}^{t} \hat{\Psi}_{n}(y)=\operatorname{Id}_{d_{1}+1}
$$

Notice that (combining Eq. (3-30) and Eq. (6-7)):

$$
\Psi_{\infty}(x)=(L(x)-R(x)) A_{n} \Psi_{n}(x)
$$

The matrix

$$
F_{n}(x):=(L(x)-R(x)) A_{n}
$$

is the so called Folding matrix of [11]. It's property is to fold any operator acting on $\Psi_{\infty}$ into an operator acting on the window only. For any finite band operator $\hat{O}$, we have:

$$
\Pi_{n}^{n-d_{2}} \hat{O} \Psi_{\infty}(x)=\left(\Pi_{n}^{n-d_{2}} \hat{O} F_{n}(x)\right) \Psi_{n}(x)
$$

$\Pi_{n}^{n-d_{2}} \hat{O} F_{n}(x)$ is a square matrix of size $d_{2}+1 \times d_{2}+1$. When acting on the window $\Psi_{n}$, it gives the same result as the operator $\hat{O}$ acting on the infinite matrix $\Psi_{\infty}$.

\section{Mixed correlation function}

We now arrive to the main results of this article, which concerns the computation of the mixed correlation function

$$
W_{n}(x, y)=1+\left\langle\operatorname{Tr} \frac{1}{x-M_{1}} \frac{1}{y-M_{2}}\right\rangle,
$$

where $M_{1}$ and $M_{2}$ are in the ensemble $H_{n} \times H_{n}(\Gamma)$ of remark 2.1. and explained in [18], with the measure:

$$
\frac{1}{Z} \mathrm{e}^{-\operatorname{Tr}\left(V_{1}\left(M_{1}\right)+V_{2}\left(M_{2}\right)+M_{1} M_{2}\right)} d M_{1} d M_{2} .
$$

and where $Z$ is the normalization constant, called partition function:

$$
Z=\int_{H_{n} \times H_{n}(\Gamma)} \mathrm{e}^{-\operatorname{Tr}\left(V_{1}\left(M_{1}\right)+V_{2}\left(M_{2}\right)+M_{1} M_{2}\right)} d M_{1} d M_{2} .
$$

We recall that $H_{n} \times H_{n}(\Gamma)$ is the set of normal matrices (i.e. $\left[M_{1}, M_{1}^{\dagger}\right]=0=\left[M_{2}, M_{2}^{\dagger}\right]$ ) with pairs of eigenvalues constrained to be on $\Gamma$, see [18] for more details. In the case $\Gamma=\mathbf{R} \times \mathbf{R}, M_{1}$ and $M_{2}$ are hermitean matrices of size $n$. 
The formula of [8] reads:

$$
W_{n}(x, y)=\operatorname{det}_{n}\left(\operatorname{Id}_{n}+\Pi_{n-1} \frac{1}{x-Q} \frac{1}{y-P^{t}} \Pi_{n-1}\right)
$$

which is a $n \times n$ determinant, and is thus not convenient for large $n$ computations, and for many other applications.

The purpose of this section is to write it in terms of a determinant of size $d_{2}+1$ or $d_{1}+1$.

\subsection{Formula for $W_{n}$}

We introduce the following lower triangular matrices ${ }^{4}$ of size $d_{1}+1\left(\right.$ resp. $\left.d_{2}+1\right)$ :

$$
\begin{array}{rlll}
U_{n}(x, y) & := & -\frac{y+V_{1}^{\prime}(x)}{\gamma_{n}} \mathbf{e}_{n} \mathbf{e}_{n-1}^{t}-\Pi_{n} \frac{V_{1}^{\prime}(x)-V_{1}^{\prime}(Q)}{x-Q} \Pi^{n-1} \\
\tilde{U}_{n}(x, y) & := & -\frac{x+V_{2}^{\prime}(y)}{\gamma_{n}} \mathbf{e}_{n} \mathbf{e}_{n-1}^{t}-\Pi_{n} \frac{V_{2}^{\prime}(y)-V_{2}^{\prime}(P)}{y-P} \Pi^{n-1}
\end{array}
$$

Many of their properties are described in appendix C.

The following theorem is proved in appendix D:

\section{Theorem 7.1}

$$
\begin{aligned}
& W_{n}(x, y) \\
& =\quad \gamma_{n}^{2} K_{n+1}(x, y) J_{n-1}(x, y) \operatorname{det}\left(\operatorname{Id}_{n}-\Pi_{n}\left(1-\frac{\psi_{\infty} \phi_{\infty}^{t}}{K_{n+1}}\right) U_{n}\left(1-\frac{\hat{\psi_{\infty}} \hat{\phi}_{\infty}^{t}}{J_{n-1}}\right) \tilde{U}_{n}^{t}\right) \\
& =\quad \gamma_{n}^{2} K_{n+1}(x, y) J_{n-1}(x, y) \operatorname{det}\left(\operatorname{Id}_{d_{2}+1}-\Pi_{n}^{n-d_{2}}\left(1-\frac{\vec{\psi}_{n} \vec{\phi}_{n}^{t}}{K_{n+1}}\right) U_{n}\left(1-\frac{\hat{\vec{\psi}}_{n} \hat{\vec{\phi}}_{n}^{t}}{J_{n-1}}\right) \tilde{U}_{n}^{t}\right) \\
& =\quad \gamma_{n}^{2} K_{n+1}(x, y) J_{n-1}(x, y) \operatorname{det}\left(\operatorname{Id}_{d_{1}+1}-\Pi_{n}^{n-d_{1}}\left(1-\frac{\hat{\vec{\psi}}_{n} \hat{\vec{\phi}}_{n}^{t}}{J_{n-1}}\right) \tilde{U}_{n}^{t}\left(1-\frac{\vec{\psi}_{n} \vec{\phi}_{n}^{t}}{K_{n+1}}\right) U_{n}\right) \\
& (7-6)
\end{aligned}
$$

or also:

$$
\begin{aligned}
W_{n}(x, y)= & \gamma_{n}^{2} \operatorname{det}\left(\operatorname{Id}_{n+1}-U_{n}(x, y) \tilde{U}_{n}(x, y)^{t}\right) \\
& \left(\left(J_{n+d_{2}}+\hat{\vec{\phi}}_{n}^{t} \frac{1}{1-\tilde{U}_{n}^{t} U_{n}} \hat{\vec{\psi}}_{n}\right)\left(K_{n-d_{2}}+\vec{\phi}_{n}^{t} \frac{1}{1-U_{n} \tilde{U}_{n}^{t}} \vec{\psi}_{n}\right)\right. \\
& \left.\quad\left(\hat{\vec{\phi}}_{n}^{t} \tilde{U}_{n}^{t} \frac{1}{1-U_{n} \tilde{U}_{n}^{t}} \vec{\psi}_{n}\right)\left(\vec{\phi}_{n}^{t} \frac{1}{1-U_{n} \tilde{U}_{n}^{t}} U_{n} \hat{\vec{\psi}}_{n}\right)\right)
\end{aligned}
$$

\footnotetext{
${ }^{4}$ They are infinite matrices, with only a non-vanishing sub-block of size $d_{1}+1$ (resp. $\left.d_{2}+1\right)$.
} 
Notice that those formulae involve only functions which are within the windows. The determinants are in fact of size $\min \left(d_{2}+1, d_{1}+1\right)$.

The proof is given in appendix D.

\section{Theorem 7.2}

$$
W_{n}(x, y)=-\tilde{t} K_{n-d_{2}}(x, y) J_{n+d_{2}}(x, y) \operatorname{det}(y-M(x, y))
$$

where $M$ is the $\left(d_{2}+1\right) \times\left(d_{2}+1\right)$ matrix

$$
M_{i j}=H_{n}^{(i j)}(x, x)+\frac{\delta_{i 0}}{K_{n-d_{2}}(x, y)}\left(y-\partial_{x}\right) K_{n-d_{2}}^{(0 j)}(x, y)+\frac{\delta_{j 0}}{J_{n+d_{2}}(x, y)}\left(y+\partial_{x}\right) J_{n+d_{2}}^{(i 0)}(x, y)
$$

\section{proof:}

We use expressions of appendix F, and Lemma I.2.

\subsection{Recursion $W_{n+1}-W_{n}$}

We introduce the following lower triangular matrices of size $d_{1}\left(\right.$ resp. $\left.d_{2}\right)$ :

$$
\mathcal{W}_{n}(x)=\Pi_{n} \frac{V_{1}^{\prime}(x)-V_{1}^{\prime}(Q)}{x-Q} \Pi^{n} \quad, \quad \tilde{\mathcal{W}}_{n}(y)=\Pi_{n} \frac{V_{2}^{\prime}(y)-V_{2}^{\prime}(P)}{y-P} \Pi^{n}
$$

The following theorem is proved in appendix E:

\section{Theorem 7.3}

$$
\begin{aligned}
& W_{n+1}(x, y)-W_{n}(x, y) \\
& =\quad K_{n+1} J_{n} \operatorname{det}\left(1-\Pi_{n}\left(1-\frac{\psi_{\infty} \phi_{\infty}^{t}}{K_{n+1}}\right) \mathcal{W}_{n}\left(1-\frac{{\hat{\psi_{\infty}}}_{\phi_{\infty}}^{t}}{J_{n}}\right) \tilde{\mathcal{W}}_{n}^{t}\right) \\
& (7-11)
\end{aligned}
$$

i.e.

$$
\begin{aligned}
& W_{n+1}(x, y)-W_{n}(x, y) \\
&=\operatorname{det}(\left.1-\mathcal{W}_{n} \tilde{\mathcal{W}}_{n}^{t}\right) \cdot\left[\left(J_{n}+\left(\hat{\phi}_{\infty}^{t} \mathcal{W}_{n}^{t} \frac{1}{1-\mathcal{W}_{n} \tilde{\mathcal{W}}_{n}^{t}} \tilde{\mathcal{W}}_{n} \hat{\psi_{\infty}}\right)\right)\left(\phi_{\infty}^{t} \frac{1}{1-\hat{\mathcal{W}}_{n} \tilde{\mathcal{W}}_{n}^{t}} \psi_{\infty}\right)\right. \\
&\left.\quad-\left(\phi_{\infty}^{t} \frac{1}{1-\mathcal{W}_{n} \tilde{\mathcal{W}}_{n}^{t}} \mathcal{W}_{n} \hat{\psi_{\infty}}\right)\left(\hat{\phi}_{\infty}^{t} \tilde{\mathcal{W}}_{n}^{t} \frac{1}{1-\mathcal{W}_{n} \tilde{\mathcal{W}}_{n}^{t}} \psi_{\infty}\right)\right]
\end{aligned}
$$

i.e. $W_{n+1}-W_{n}$ actualy involves the computation of a determinant and inverse of a matrix of size $\min \left(d_{1}, d_{2}\right)$.

The proof is given in appendix E. 


\section{Application: differential systems and spectral curve}

An important observation of [9] and [10, 32, 29] is that windows of consecutive biorthogonal polynomials satisfy some integrable differential systems, and a RiemannHilbert problem. An explicit representation of those differential systems was found in 11. Here, thanks to the result for the mixed correlation function, we are able to give new representations of those systems, and compute explicitely their spectral curve.

\subsection{Differential systems}

We define the following $\left(d_{2}+1\right) \times\left(d_{2}+1\right)$ and $\left(d_{1}+1\right) \times\left(d_{1}+1\right)$ matrices:

$$
\begin{array}{lll}
\mathcal{D}_{n}(x):=\Psi_{n}^{\prime}(x) \Psi_{n}^{-1}(x) \quad & \hat{\mathcal{D}}_{n}(x):=-\hat{\Phi}_{n}^{\prime}(x) \hat{\Phi}_{n}^{-1}(x) \\
\tilde{\mathcal{D}}_{n}(y):=\Phi_{n}^{\prime}(y) \Phi_{n}^{-1}(y) & , & \hat{\tilde{\mathcal{D}}}_{n}(y):=-\hat{\Psi}_{n}^{\prime}(y) \hat{\Psi}_{n}^{-1}(y) .
\end{array}
$$

Those matrices are square matrices of the size of the corresponding window, and they have polynomial entries. They give some ODE's for the windows:

$$
\Psi_{n}^{\prime}(x)=\mathcal{D}_{n}(x) \Psi_{n}(x)
$$

It was found in 9] that they enjoy some duality relations (which merely come from duality Eq. (6-7) $)$ :

$$
\hat{\mathcal{D}}_{n}^{t}(x) A_{n}=A_{n} \mathcal{D}_{n}(x) \quad, \quad \hat{\tilde{\mathcal{D}}}_{n}^{t}(y) B_{n}=B_{n} \tilde{\mathcal{D}}_{n}(y) .
$$

Theorem 8.1 We have the following equivalent expressions for $\mathcal{D}$ :

$$
\begin{array}{lll}
\mathcal{D}_{n}(x) & =\Pi_{n}^{n-d_{2}} P^{t}(L(x)-R(x)) A_{n} \\
y-\mathcal{D}_{n}(x) & =\left(\tilde{U}_{n}^{t-1}(x, y)-\Pi_{n}^{n-d_{2}} U_{n}(x, y)\right) A_{n} \\
\left(\Psi_{n}^{-1}(x) \mathcal{D}_{n}(x) \Psi_{n}(x)\right)_{i j} & =H^{(i j)}(x, x)
\end{array}
$$

And the matrix elements of $y-\mathcal{D}_{n}(x)$ are $\left(d_{1}+1\right) \times\left(d_{1}+1\right)$ determinants given by $\left(0 \leq k, l \leq d_{2}\right)$ :

- If $k \geq d_{1}$

$$
\operatorname{det}\left(\begin{array}{cc}
\mathbf{e}_{n-k}^{t}\left(y-P_{n}\right) \Pi_{n-d_{2}-1}^{n-d_{1}-d_{2}} & \frac{1}{\prod_{m=1}^{d_{1}} Q_{n-m, n-m-d_{2}}} \\
\mathbf{e}_{n-k}^{t}\left(y-P^{t}\right) \mathbf{e}_{n-l} \\
\prod_{n-1}^{n-d_{1}}(x-Q) \Pi_{n-d_{2}-1}^{n-d_{1}-d_{2}} & \Pi_{n-1}^{n-d_{1}}(x-Q) \mathbf{e}_{n-l}
\end{array}\right)
$$




$$
\begin{aligned}
& \text { - If } k<d_{1} \\
& \begin{array}{c}
\left(y-\mathcal{D}_{n}(x)\right)_{n-k, n-l}=\frac{(-1)^{d_{1}-k}}{\prod_{m=0}^{d_{1}-k} \gamma_{n+m-1} \prod_{m=1}^{k} Q_{n-m, n-m-d_{2}}} \times \operatorname{det} \\
\left(\begin{array}{ccc}
\mathbf{e}_{n-k}^{t}\left(y-P^{t}\right) \Pi_{n-d_{2}-1}^{n-d_{2}-k} & \mathbf{e}_{n-k}^{t}\left(y-P^{t}\right) \mathbf{e}_{n-l} & \mathbf{e}_{n-k}^{t}\left(y-P^{t}\right) \Pi_{n+d_{1}-k}^{n+1} \\
\prod_{n+d_{1}-k-1}^{n-k}(x-Q) \Pi_{n-d_{2}-1}^{n-d_{2}-k} & \prod_{n+d_{1}-k-1}^{n-k}(x-Q) \mathbf{e}_{n-l} & \prod_{n+d_{1}-k-1}^{n-k}(x-Q) \Pi_{n+d_{1}-k}^{n+1}
\end{array}\right)
\end{array}
\end{aligned}
$$

The first equality of Eq. (8-5) was found in [11, it comes from the Folding matrix Eq. (6-10): $\Psi_{n}^{\prime}=\Pi_{n}^{n-d_{2}} \Psi_{\infty}^{\prime}=\Pi_{n}^{n-d_{2}} P^{t} \Psi_{\infty}=\Pi_{n}^{n-d_{2}} P^{t} F_{n}(x) \Psi_{n}$.

The second equality is a rewriting of the first one using the matrices $U_{n}$ and $\tilde{U}_{n}$, see appendix C.

The third equality is a mere rewriting of the definition of $\mathcal{D}$, using the duality Eq. (4-30).

The last representation is obtained by using the first formula, and computing the folding matrix by inverting the linear problem $\Psi_{\infty}=F_{n} \Psi_{n}$ in the kernel of $x-Q$.

\subsection{Spectral curve}

It was proven in [9] that all those differential systems share the same spectral curve. We define:

$$
\begin{aligned}
\mathcal{E}_{n}(x, y) & :=\quad \tilde{t} \operatorname{det}\left(y-\mathcal{D}_{n}(x)\right)=\tilde{t} \operatorname{det}\left(y-\hat{\mathcal{D}}_{n}(x)\right) \\
& =\quad t \operatorname{det}\left(x-\tilde{\mathcal{D}}_{n}(y)\right)=t \operatorname{det}\left(x-\tilde{\hat{\mathcal{D}}}_{n}(y)\right) \\
(8-8) &
\end{aligned}
$$

We are going to give here several equivalent formulae for computing $\mathcal{E}$.

Theorem 8.2 We have:

$$
\mathcal{E}_{n}(x, y)=-\gamma_{n}^{2} \operatorname{det}\left(1-U_{n} \tilde{U}_{n}^{t}\right)
$$

and

$$
\mathcal{E}_{n+1}(x, y)-\mathcal{E}_{n}(x, y)=-\operatorname{det}\left(1-\mathcal{W}_{n} \tilde{\mathcal{W}}_{n}^{t}\right)
$$

proof:

For the first expression, use the 2nd expression in theorem 8.1. The proof of the recursion formula is found in appendix $\mathrm{H}$.

Then, we introduce the following Lemma, which consists in taking the polynomial part at large $y$ of formula Eq. (7-7): 


\section{Lemma 8.1}

$$
\underset{y \rightarrow \infty}{\operatorname{Pol}}\left(V_{2}^{\prime}(y)+x\right) W_{n}(x, y)=\tilde{t} \operatorname{det}_{i, j \neq 0}\left(y-H^{(i j)}\right)
$$

i.e.

$$
V_{2}^{\prime}(y)+x+\left\langle\operatorname{Tr} \frac{1}{x-M_{1}} \frac{V_{2}^{\prime}(y)-V_{2}^{\prime}\left(M_{2}\right)}{y-M_{2}}\right\rangle=\tilde{t} \operatorname{det}_{i, j \neq 0}\left(y-H^{(i j)}\right)
$$

Notice that this formula is the finite $n$ counterpart of what was found in the formal large $n$ expansion in [14, namely theorem 3.1, equation (3.3) of [14].

proof:

This Lemma is proved in appendix F.5.

Using this Lemma, taking the polynomial part in $x$ at large $x$, we prove the following theorem, which was conjectured by Marco Bertola [5]:

Theorem 8.3 Proof of Bertola's conjecture:

$$
\underset{x \rightarrow \infty}{\operatorname{Pol}} \operatorname{Pol}\left(V_{1}^{\prime}(x)+y\right)\left(V_{2}^{\prime}(y)+x\right) W_{n}(x, y)=2 n+\mathcal{E}_{n}(x, y)
$$

i.e.

$$
\mathcal{E}_{n}(x, y)=\left(V_{1}^{\prime}(x)+y\right)\left(V_{2}^{\prime}(y)+x\right)+\left\langle\operatorname{Tr} \frac{V_{1}^{\prime}(x)-V_{1}^{\prime}\left(M_{1}\right)}{x-M_{1}} \frac{V_{2}^{\prime}(y)-V_{2}^{\prime}\left(M_{2}\right)}{y-M_{2}}\right\rangle-n
$$

Marco Bertola has proved it for potentials of degree $\max \left(d_{1}, d_{2}\right) \leq 5$, which is quite remarkable, and also for the smallest values of $n$ [5].

This theorem is also the finite $n$ counterpart of theorem 3.1 of [14. Notice that the spectral curve for finite $n$ is the same as the algebraic curve found from large $n$ considerations 14. This fact has important consequences, some of which are described below in the next section.

\section{proof:}

The proof is in appendix $\mathrm{G}$.

For completeness, we also write a formula for the spectral curve, due to Jacques Hurtubise:

Theorem 8.4 Hurtubise formula [26]

$$
\mathcal{E}_{n}(x, y)=-\frac{\gamma_{n}}{\prod_{j=n-d_{2}}^{n+d_{1}} \gamma_{j}} \operatorname{det}\left(\begin{array}{c}
\prod_{n}^{n-d_{2}}\left(P^{t}-y\right) \\
\prod_{n+d_{1}-1}^{n-1}(Q-x)
\end{array}\right)
$$


which simply amounts to say that if $\mathcal{E}_{n}(x, y)=0$, there must exist some functions $\psi_{n-d_{2}-1}, \ldots, \psi_{n+d_{1}}$, such that we have simultaneously $y \psi_{i}(x)=\psi_{i}^{\prime}(x)=\sum_{i} P_{j i} \psi_{j}(x)$ for $i=n-d_{2}, \ldots, n$ and $x \psi_{i}(x)=\sum_{i} Q_{i j} \psi_{j}(x)$ for $i=n-1, \ldots, n+d_{1}-1$, i.e. the vector $\left(\psi_{n-d_{2}-1}, \ldots, \psi_{n+d_{1}}\right)^{t}$ is in the kernel of the matrix above.

\section{$9 \quad$ Examples}

\subsection{Gaussian case, Ginibre polynomials}

Consider $V_{1}(x)=V_{2}(y)=0$, which is Ginibre's ensemble. It is well known that the bi-orthogonal polynomials are monomials [24]:

$$
p_{n}(x)=x^{n} \quad, \quad q_{n}(y)=y^{n} \quad, \quad h_{n}=n ! \pi
$$

and the Cauchy transforms are

$$
\hat{p}_{n}(y)=\frac{\pi n !}{y^{n+1}} \quad, \quad \hat{q}_{n}(x)=\frac{\pi n !}{x^{n+1}} .
$$

We have:

$$
Q_{n m}=P_{n m}=\sqrt{n+1} \delta_{m, n+1} \quad, \quad \gamma_{n}=\sqrt{n}
$$

We find that for $n>m$ :

$$
R_{n m}(x)=-\psi_{n}(x) \hat{\phi}_{m}(x),
$$

and $\frac{1}{x-Q}$ is an upper triangular matrix.

In that case, theorem 7.1 becomes:

$$
\begin{aligned}
W_{n}(x, y) & =1+n J_{n-1}(x, y) K_{n}(x, y)-x y J_{n}(x, y) K_{n-1}(x, y) \\
& =n K_{n+1} J_{n-1}-x y K_{n} J_{n},
\end{aligned}
$$

and theorem (7.3) with $\mathcal{W}_{n}=0$ becomes:

$$
W_{n+1}-W_{n}=K_{n+1} J_{n}
$$

\subsection{Gaussian elliptical case}

Consider $V_{1}^{\prime}(x)=t x, V_{2}^{\prime}(y)=\tilde{t} y$, we write:

$$
\delta=1-t \tilde{t}
$$

The bi-orthogonal polynomials are rescaled Hermite polynomials [33], and we have:

$$
\gamma_{n}=Q_{n-1, n}=P_{n-1, n}=\sqrt{\frac{n}{\delta}}
$$




$$
Q_{n, n-1}=-\tilde{t} \gamma_{n} \quad, \quad P_{n, n-1}=-t \gamma_{n}
$$

The Christoffel-Darboux matrices are:

$$
A_{n}=\gamma_{n}\left(\begin{array}{cc}
0 & 1 \\
\tilde{t} & 0
\end{array}\right) \quad, \quad B_{n}=\gamma_{n}\left(\begin{array}{ll}
0 & 1 \\
t & 0
\end{array}\right)
$$

The matrices $U_{n}, \tilde{U}_{n}$ are:

$$
U_{n}(x, y)=-\left(\begin{array}{cc}
t & 0 \\
\frac{y+t x}{\gamma_{n}} & t
\end{array}\right) \quad, \quad \tilde{U}_{n}^{t}(x, y)=-\left(\begin{array}{cc}
\tilde{t} & \frac{x+\tilde{t} y}{\gamma_{n}} \\
0 & \tilde{t}
\end{array}\right)
$$

and the matrices $\mathcal{W}_{n}$ and $\tilde{\mathcal{W}}_{n}$ are of dimension 1 :

$$
\mathcal{W}(x)=t \quad, \quad \tilde{\mathcal{W}}(y)=\tilde{t}
$$

The differential systems are:

$$
\begin{aligned}
& \mathcal{D}_{n}(x)=\left(\begin{array}{cc}
-\frac{x}{t} & \frac{\delta}{t} \gamma_{n} \\
\delta \gamma_{n} & -t x
\end{array}\right) \quad, \quad \overline{\mathcal{D}}_{n}(x)=\left(\begin{array}{cc}
-t x & \delta \gamma_{n} \\
\frac{\delta}{t} \gamma_{n} & -\frac{x}{t}
\end{array}\right) \\
& \tilde{\mathcal{D}}_{n}(y)=\left(\begin{array}{cc}
-\frac{y}{t} & \frac{\delta}{t} \gamma_{n} \\
\delta \gamma_{n} & -\tilde{t} y
\end{array}\right) \quad, \quad \overline{\mathcal{D}}_{n}(y)=\left(\begin{array}{cc}
-\tilde{t} y & \delta \gamma_{n} \\
\frac{\delta}{t} \gamma_{n} & -\frac{y}{t}
\end{array}\right)
\end{aligned}
$$

thus the spectral curve is:

$$
\mathcal{E}_{n}(x, y)=\tilde{t} \operatorname{det}\left(y-\mathcal{D}_{n}(x)\right)=(x+\tilde{t} y)(y+t x)-\delta n=-\gamma_{n}^{2} \operatorname{det}\left(1-U_{n} \tilde{U}_{n}^{t}\right)
$$

Theorem 7.3 gives:

$$
W_{n+1}-W_{n}=J_{n} K_{n+1}-t \tilde{t} J_{n+1} K_{n}
$$

\section{Tau-function}

Theorem 8.3 implies for $k \leq d_{1}$ and $l \leq d_{2}$ :

$$
\left\langle\operatorname{Tr} M_{1}^{k} M_{2}^{l}\right\rangle=\operatorname{Res}_{x \rightarrow \infty} \operatorname{Res}_{y \rightarrow \infty} \frac{\mathcal{E}_{n}(x, y) x^{k} y^{l}}{V_{1}^{\prime}(x) V_{2}^{\prime}(y)} d x d y
$$

Thus, if we introduce the matrix integral:

$$
\tilde{Z}=\int d M_{1} d M_{2} \mathrm{e}^{-\left[\sum_{k, l} t_{k, l} \operatorname{Tr} M_{1}^{k} M_{2}^{l}\right]}
$$

we have:

$$
\operatorname{Res}_{x \rightarrow \infty} \operatorname{Res}_{y \rightarrow \infty} \frac{\mathcal{E}_{n}(x, y) x^{k} y^{l}}{V_{1}^{\prime}(x) V_{2}^{\prime}(y)} d x d y=-\left.\frac{\partial \ln \tilde{Z}}{\partial t_{k, l}}\right|_{t_{k, l}=0 \text { if } k l>1}
$$


Equation Eq. (10-3) is similar to the tau-function of [28, 27, 36, 22] generalized to the resonant case by [7, and thus, similarly to [12, we have proved that:

$$
\tau\left(\left\{t_{k, l}\right\}\right)=\tilde{Z} \quad \text { for } t_{k, l}=0 \text { if } k l>1
$$

This strongly suggests that the general setting of the isomonodromic problem should include all $t_{k, l}$ 's.

It is easy to define bi-orthogonal polynomials with a weight of the form

$$
\omega(x, y)=\mathrm{e}^{-\sum_{k, l} t_{k, l} x^{k} y^{l}}
$$

and they are related to the so called ensemble of normal complex matrix integrals (see 38 ):

$$
Z_{\text {normal }}=\int_{N_{n}} d M \mathrm{e}^{-\sum_{k, l} t_{k, l} \operatorname{Tr} M^{k} M^{\dagger l}}
$$

where $N_{n}$ is the set of complex matrices which commute with their adjoint $\left[M, M^{\dagger}\right]=0$ (they have complex eigenvalues not constrained on any path). For normal matrices, we have:

$$
\left\langle\operatorname{Tr} \frac{1}{x-M} \frac{1}{y-M^{\dagger}}\right\rangle=\operatorname{Tr}\left(\Pi_{n-1} \frac{1}{y-P^{t}} \frac{1}{x-Q} \Pi_{n-1}\right)
$$

so that our $W_{n}$ is not the mixed correlation function for normal matrices.

Our $W_{n}(x, y)$ is in that model:

$$
W_{n}(x, y)=\left\langle\operatorname{det}\left(1+\frac{1}{x-M} \frac{1}{y-M^{\dagger}}\right)\right\rangle=\operatorname{det}\left(1+\Pi_{n-1} \frac{1}{x-Q} \frac{1}{y-P^{t}} \Pi_{n-1}\right) .
$$

It is not clear yet why this function should be related to the spectral curve for the normal matrix model...

\section{Conclusion}

In this article, we have found a formula for computing the mixed correlation function as a $\min \left(d_{1}+1, d_{2}+1\right)$ determinant, instead of a $n \times n$ determinant. This kind of formula can be very useful for finding large $n$ limits. Beside, we have found several expressions for the spectral curve, which can be useful for studying the integrability properties. In particular, we have proved the conjecture of Marco Bertola, which should open the route to other applications.

In this article we have computed the 2-point mixed correlation function, however, for many applications to physics (BMN limit of ADS/CFT correspondance in string theory, or boundary conformal field theory), one needs mixed correlation functions involving more than one trace, and more than two matrices in a trace. The key element 
of the work of [8], was the use of Morozov's formula for 2-point correlation functions of unitary integrals. Since then, some generalizations of Morozov's formula have been found for arbitrary correlation functions [21], and it seems natural to generalize the result of [8], and of the present paper. In that prospect, one could expect to understand the Bethe-ansatz-like structure of large $n$ mixed correlation, which was found in [20].

\section{Aknowledgements}

We would like to thank Marco Bertola, John Harnad, Jacques Hurtubise and Ken Mc Laughlin for fruitful discussions on those subjects. Our work is partly supported by the Enigma european network MRT-CT-2004-5652, by the ANR project Géométrie et intégrabilité en physique mathématique ANR-05-BLAN-0029-01, and by the Enrage european network MRTN-CT-2004-005616. B.E. thanks the CRM and the BIRS for their supports. 


\section{Appendix A Proof of theorem 3.1}

proof:

Notice that $\hat{\psi}_{n}(y)$ is piecewise analytical in the connected domains separated by the $\tilde{\gamma}^{(i)}$ 's. In each such domain, it bahaves at $\infty$, like:

$$
\hat{\psi}_{n}(y) \sim_{\infty} \sqrt{h_{n}} y^{-n-1} \mathrm{e}^{V_{2}(y)}(1+O(1 / y))
$$

Let $f_{n}(x, y)$ be the primitive of $\hat{\psi}_{n}(y) \mathrm{e}^{x y}$ which vanishes at $\infty$ in sectors where $\operatorname{Re} V_{2}<0$ :

$$
f_{n}(x, y)=\int^{y} \hat{\psi}_{n}\left(y^{\prime \prime}\right) \mathrm{e}^{x y^{\prime \prime}}
$$

$f_{n}(x, y)$ behaves at $\infty$ in each connected domain, like:

$$
f_{n}(x, y) \sim_{\infty} \frac{1}{\tilde{t}} \sqrt{h_{n}} y^{-n-d_{2}-1} \mathrm{e}^{V_{2}(y)+x y}\left(1+\sum_{k=1}^{\infty} f_{n, k}(x) y^{-k}\right)
$$

where each $f_{n, k}(x)$ is a polynomial in $x$.

By definition of $L_{n m}(x)$ we have:

$$
\begin{aligned}
L_{n m}(x) & =\frac{1}{2 i \pi} \sum_{i=1}^{d_{2}} \int_{\tilde{\gamma}^{(i)}} d y \phi_{m}(y) \mathrm{e}^{-x y}\left(f_{n}\left(x, y_{+}\right)-f_{n}\left(x, y_{-}\right)\right) \\
& =\frac{1}{2 i \pi} \sum_{\text {domains } D} \int_{\partial D} d y \phi_{m}(y) \mathrm{e}^{-x y} f_{n}(x, y) \\
& =\frac{1}{2 i \pi} \sum_{\text {domains } D} \int_{\partial D} d y q_{m}(y) y^{-n-d_{2}-1} \mathrm{e}^{-x y}\left(1+\sum_{k=1}^{\infty} f_{n, k}(x) y^{-k}\right) \\
& =\operatorname{Res}_{\infty} d y q_{m}(y) y^{-n-d_{2}-1} \mathrm{e}^{-x y}\left(1+\sum_{k=1}^{\infty} f_{n, k}(x) y^{-k}\right) \\
(1-4) &
\end{aligned}
$$

This expression vanishes if $m<n+d_{2}$, and is clearly a polynomial in $x$.

Now, compute:

$$
\begin{aligned}
& \sum_{i=1}^{d_{2}} \psi_{n}^{(i)}(x) \hat{\phi}_{m}^{(i)}(x) \\
= & \frac{1}{2 i \pi \sqrt{h_{n} h_{m}}} \sum_{i=1}^{d_{2}} \int_{\tilde{\gamma}^{(i)}} d y \int_{\tilde{\bar{\gamma}}^{(i)}} d y^{\prime \prime} \int_{\Gamma} d x^{\prime} d y^{\prime} \mathrm{e}^{-V_{2}(y)} \mathrm{e}^{V_{2}\left(y^{\prime \prime}\right)} \mathrm{e}^{-V_{2}\left(y^{\prime}\right)} \mathrm{e}^{-V_{1}\left(x^{\prime}\right)} \\
& \frac{1}{x-x^{\prime}} \frac{V_{2}^{\prime}\left(y^{\prime \prime}\right)-V_{2}^{\prime}\left(y^{\prime}\right)}{y^{\prime \prime}-y^{\prime}} p_{n}\left(x^{\prime}\right) q_{m}(y) \mathrm{e}^{x y^{\prime \prime}} \mathrm{e}^{-x y} \mathrm{e}^{-x^{\prime} y^{\prime}} \\
= & \sum_{i=1}^{d_{2}} \sum_{j, k} \frac{\kappa_{k j}}{2 i \pi \sqrt{h_{n} h_{m}}} \int_{\tilde{\gamma}^{(i)}} d y \int_{\tilde{\gamma}^{(i)}} d y^{\prime \prime} \int_{\gamma^{(k)}} d x^{\prime} \int_{\tilde{\gamma}^{(j)}} d y^{\prime} \mathrm{e}^{-V_{2}(y)} \mathrm{e}^{V_{2}\left(y^{\prime \prime}\right)} \mathrm{e}^{-V_{2}\left(y^{\prime}\right)} \mathrm{e}^{-V_{1}\left(x^{\prime}\right)}
\end{aligned}
$$




$$
\begin{aligned}
& \frac{1}{x-x^{\prime}} \frac{V_{2}^{\prime}\left(y^{\prime \prime}\right)-V_{2}^{\prime}\left(y^{\prime}\right)}{y^{\prime \prime}-y^{\prime}} p_{n}\left(x^{\prime}\right) q_{m}(y) \mathrm{e}^{x y^{\prime \prime}} \mathrm{e}^{-x y} \mathrm{e}^{-x^{\prime} y^{\prime}} \\
= & \sum_{i=1}^{d_{2}} \sum_{j, k} \frac{\kappa_{k j}}{2 i \pi \sqrt{h_{n} h_{m}}} \int_{\tilde{\gamma}^{(i)}} d y \int_{\tilde{\bar{\gamma}}_{+}^{(i)}(y) \cup \tilde{\bar{\gamma}}_{-}^{(i)}(y)} d y^{\prime \prime} \int_{\gamma^{(k)}} d x^{\prime} \int_{\tilde{\gamma}^{(j)}} d y^{\prime} \\
& \mathrm{e}^{-V_{2}(y)} \mathrm{e}^{V_{2}\left(y^{\prime \prime}\right)} \mathrm{e}^{-V_{2}\left(y^{\prime}\right)} \mathrm{e}^{-V_{1}\left(x^{\prime}\right)} \frac{1}{x-x^{\prime}} \frac{V_{2}^{\prime}\left(y^{\prime \prime}\right)-V_{2}^{\prime}\left(y^{\prime}\right)}{y^{\prime \prime}-y^{\prime}} p_{n}\left(x^{\prime}\right) q_{m}(y) \mathrm{e}^{x y^{\prime \prime}} \mathrm{e}^{-x y} \mathrm{e}^{-x^{\prime} y^{\prime}}
\end{aligned}
$$

Moreover we have:

$$
\begin{aligned}
& =\frac{1}{2 i \pi} \int_{\tilde{\bar{\gamma}}_{+}^{(i)}(y) \cup \tilde{\bar{\gamma}}_{-}^{(i)}(y)} d y^{\prime \prime} \int_{\tilde{\gamma}^{(j)}} d y^{\prime} \mathrm{e}^{V_{2}\left(y^{\prime \prime}\right)} \mathrm{e}^{-V_{2}\left(y^{\prime}\right)} \frac{V_{2}^{\prime}\left(y^{\prime \prime}\right)-V_{2}^{\prime}\left(y^{\prime}\right)}{y^{\prime \prime}-y^{\prime}} \mathrm{e}^{x y^{\prime \prime}} \mathrm{e}^{-x^{\prime} y^{\prime}} \\
& =\frac{1}{2 i \pi} \int_{\tilde{\bar{\gamma}}_{+}^{(i)}(y) \cup \tilde{\bar{\gamma}}_{-}^{(i)}(y)} d y^{\prime \prime} \int_{\tilde{\gamma}^{(j)}} d y^{\prime} \frac{V_{2}^{\prime}\left(y^{\prime \prime}\right)}{y^{\prime \prime}-y^{\prime}} \mathrm{e}^{V_{2}\left(y^{\prime \prime}\right)} \mathrm{e}^{-V_{2}\left(y^{\prime}\right)} \mathrm{e}^{-x^{\prime} y^{\prime}} \mathrm{e}^{x y^{\prime \prime}} \\
& -\frac{1}{2 i \pi} \int_{\tilde{\bar{\gamma}}_{+}^{(i)}(y) \cup \tilde{\bar{\gamma}}_{-}^{(i)}(y)} d y^{\prime \prime} \int_{\tilde{\gamma}^{(j)}} d y^{\prime} \frac{V_{2}^{\prime}\left(y^{\prime}\right)}{y^{\prime \prime}-y^{\prime}} \mathrm{e}^{V_{2}\left(y^{\prime \prime}\right)} \mathrm{e}^{-V_{2}\left(y^{\prime}\right)} \mathrm{e}^{-x^{\prime} y^{\prime}} \mathrm{e}^{x y^{\prime \prime}} \\
& =\frac{1}{2 i \pi} \int_{\tilde{\bar{\gamma}}_{+}^{(i)}(y) \cup \tilde{\bar{\gamma}}_{-}^{(i)}(y)} d y^{\prime \prime} \int_{\tilde{\gamma}^{(j)}} d y^{\prime} \frac{1}{\left(y^{\prime \prime}-y^{\prime}\right)^{2}} \mathrm{e}^{V_{2}\left(y^{\prime \prime}\right)} \mathrm{e}^{-V_{2}\left(y^{\prime}\right)} \mathrm{e}^{-x^{\prime} y^{\prime}} \mathrm{e}^{x y^{\prime \prime}} \\
& -\frac{1}{2 i \pi} \int_{\tilde{\bar{\gamma}}_{+}^{(i)}(y) \cup \tilde{\bar{\gamma}}_{-}^{(i)}(y)} d y^{\prime \prime} \int_{\tilde{\gamma}^{(j)}} d y^{\prime} \frac{x}{y^{\prime \prime}-y^{\prime}} \mathrm{e}^{V_{2}\left(y^{\prime \prime}\right)} \mathrm{e}^{-V_{2}\left(y^{\prime}\right)} \mathrm{e}^{-x^{\prime} y^{\prime}} \mathrm{e}^{x y^{\prime \prime}} \\
& -\frac{\delta_{i j}}{2 i \pi} \int_{\tilde{\gamma}^{(j)}} d y^{\prime}\left[\frac{1}{y^{\prime \prime}-y^{\prime}} \mathrm{e}^{V_{2}\left(y^{\prime \prime}\right)} \mathrm{e}^{-V_{2}\left(y^{\prime}\right)} \mathrm{e}^{-x^{\prime} y^{\prime}} \mathrm{e}^{x y^{\prime \prime}}\right] \\
& -\frac{1}{2 i \pi} \int_{\tilde{\bar{\gamma}}_{+}^{(i)}(y) \cup \tilde{\bar{\gamma}}_{-}^{(i)}(y)} d y^{\prime \prime} \int_{\tilde{\gamma}^{(j)}} d y^{\prime} \frac{1}{\left(y^{\prime \prime}-y^{\prime}\right)^{2}} \mathrm{e}^{V_{2}\left(y^{\prime \prime}\right)} \mathrm{e}^{-V_{2}\left(y^{\prime}\right)} \mathrm{e}^{-x^{\prime} y^{\prime}} \mathrm{e}^{x y^{\prime \prime}} \\
& +\frac{1}{2 i \pi} \int_{\tilde{\bar{\gamma}}_{+}^{(i)}(y) \cup \tilde{\bar{\gamma}}_{-}^{(i)}(y)} d y^{\prime \prime} \int_{\tilde{\gamma}^{(j)}} d y^{\prime} \frac{x^{\prime}}{y^{\prime \prime}-y^{\prime}} \mathrm{e}^{V_{2}\left(y^{\prime \prime}\right)} \mathrm{e}^{-V_{2}\left(y^{\prime}\right)} \mathrm{e}^{-x^{\prime} y^{\prime}} \mathrm{e}^{x y^{\prime \prime}} \\
& =\quad \delta_{i j} \operatorname{Res}_{y} d y^{\prime} \frac{1}{y-y^{\prime}} \mathrm{e}^{V_{2}(y)} \mathrm{e}^{-V_{2}\left(y^{\prime}\right)} \mathrm{e}^{-x^{\prime} y^{\prime}} \mathrm{e}^{x y} \\
& -\frac{1}{2 i \pi} \int_{\tilde{\bar{\gamma}}_{+}^{(i)}(y) \cup \tilde{\bar{\gamma}}_{-}^{(i)}(y)} d y^{\prime \prime} \int_{\tilde{\gamma}^{(j)}} d y^{\prime} \frac{x-x^{\prime}}{y^{\prime \prime}-y^{\prime}} \mathrm{e}^{V_{2}\left(y^{\prime \prime}\right)} \mathrm{e}^{-V_{2}\left(y^{\prime}\right)} \mathrm{e}^{-x^{\prime} y^{\prime}} \mathrm{e}^{x y^{\prime \prime}} \\
& =\quad-\delta_{i j} \mathrm{e}^{\left(x-x^{\prime}\right) y} \\
& -\frac{1}{2 i \pi} \int_{\tilde{\bar{\gamma}}_{+}^{(i)}(y) \cup \tilde{\bar{\gamma}}_{-}^{(i)}(y)} d y^{\prime \prime} \int_{\tilde{\gamma}^{(j)}} d y^{\prime} \frac{x-x^{\prime}}{y^{\prime \prime}-y^{\prime}} \mathrm{e}^{V_{2}\left(y^{\prime \prime}\right)} \mathrm{e}^{-V_{2}\left(y^{\prime}\right)} \mathrm{e}^{-x^{\prime} y^{\prime}} \mathrm{e}^{x y^{\prime \prime}}
\end{aligned}
$$

Therefore:

$$
\begin{aligned}
& \sum_{i=1}^{d_{2}} \psi_{n}^{(i)}(x) \hat{\phi}_{m}^{(i)}(x) \\
= & -\sum_{i=1}^{d_{2}} \sum_{j, k} \frac{\kappa_{k j} \delta_{i j}}{\sqrt{h_{n} h_{m}}} \int_{\tilde{\gamma}^{(i)}} d y \int_{\gamma^{(k)}} d x^{\prime} \mathrm{e}^{-V_{2}(y)} \mathrm{e}^{-V_{1}\left(x^{\prime}\right)} \frac{1}{x-x^{\prime}} p_{n}\left(x^{\prime}\right) q_{m}(y) \mathrm{e}^{-x^{\prime} y} \\
& -\sum_{i=1}^{d_{2}} \sum_{j, k} \frac{\kappa_{k j}}{2 i \pi \sqrt{h_{n} h_{m}}} \int_{\tilde{\gamma}^{(i)}} d y \int_{\tilde{\bar{\gamma}}_{+}^{(i)}(y) \cup \tilde{\gamma}_{-}^{(i)}(y)} d y^{\prime \prime} \int_{\gamma^{(k)}} d x^{\prime} \int_{\tilde{\gamma}^{(j)}} d y^{\prime}
\end{aligned}
$$




$$
\begin{aligned}
& \mathrm{e}^{-V_{2}(y)} \mathrm{e}^{V_{2}\left(y^{\prime \prime}\right)} \mathrm{e}^{-V_{2}\left(y^{\prime}\right)} \mathrm{e}^{-V_{1}\left(x^{\prime}\right)} \frac{1}{y^{\prime \prime}-y^{\prime}} p_{n}\left(x^{\prime}\right) q_{m}(y) \mathrm{e}^{x y^{\prime \prime}} \mathrm{e}^{-x y} \mathrm{e}^{-x^{\prime} y^{\prime}} \\
= & -\left(\frac{1}{x-Q}\right)_{n m} \\
& -\sum_{i=1}^{d_{2}} \sum_{j, k} \frac{\kappa_{k j}}{2 i \pi \sqrt{h_{n} h_{m}}} \int_{\tilde{\gamma}^{(i)}} d y \int_{\tilde{\bar{\gamma}}_{+}^{(i)}(y) \cup \tilde{\bar{\gamma}}_{-}^{(i)}(y)} d y^{\prime \prime} \int_{\gamma^{(k)}} d x^{\prime} \int_{\tilde{\gamma}^{(j)}} d y^{\prime} \\
= & -\left(\frac{1}{x-Q}\right)_{n m}-\sum_{i=1}^{-V_{2}(y)} \frac{1}{2 i \pi} \int_{\tilde{\gamma}^{(i)}} d y \int_{\tilde{\bar{\gamma}}_{+}^{(i)}(y) \cup \tilde{\bar{\gamma}}_{-}^{(i)}(y)} \mathrm{e}^{-V_{2}\left(y^{\prime}\right)} \mathrm{e}^{-V_{1}\left(x^{\prime}\right)} \frac{1}{y^{\prime \prime}-y^{\prime}} p_{n}\left(x^{\prime}\right) q_{m}(y) \mathrm{e}^{x y^{\prime \prime}} \mathrm{e}^{-x y} \mathrm{e}^{-x^{\prime} y^{\prime}} \\
= & \left.-\left(\frac{1}{x-Q}\right)_{n m}+L_{n m}^{\prime \prime}\right) \phi_{m}(x) \mathrm{e}^{x y^{\prime \prime}} \mathrm{e}^{-x y}
\end{aligned}
$$

Using Eq. (2-52), this also proves that $L$ is a left inverse of $x-Q$.

\section{Appendix B Proof of determinantal formulae}

\section{B.1 Inverses}

1.

$$
\begin{aligned}
& \left(\Pi_{n-1}\left(1-\frac{\psi_{\infty} \mathbf{e}_{n}^{t}}{\psi_{n}}\right) \Pi_{n} R \Pi_{n-1}\right) \cdot\left(\Pi_{n-1}(x-Q) \Pi_{n-1}\right) \\
= & \Pi_{n-1}\left(1-\frac{\psi_{\infty} \mathbf{e}_{n}^{t}}{\psi_{n}}\right) \Pi_{n} R(x-Q) \Pi_{n-1} \\
= & \Pi_{n-1}\left(1-\frac{\psi_{\infty} \mathbf{e}_{n}^{t}}{\psi_{n}}\right) \Pi_{n}\left(1-\frac{\psi_{\infty} \mathbf{e}_{0}^{t}}{\psi_{0}}\right) \Pi_{n-1} \\
= & \Pi_{n-1}\left(1-\frac{\psi_{\infty} \mathbf{e}_{n}^{t}}{\psi_{n}}-\frac{\psi_{\infty} \mathbf{e}_{0}^{t}}{\psi_{0}}+\frac{\psi_{\infty} \mathbf{e}_{n}^{t}}{\psi_{n}} \frac{\psi_{\infty} \mathbf{e}_{0}^{t}}{\psi_{0}}\right) \Pi_{n-1} \\
= & \Pi_{n-1}\left(1-\frac{\psi_{\infty} \mathbf{e}_{n}^{t}}{\psi_{n}}\right) \Pi_{n-1} \\
= & \operatorname{Id}_{n}
\end{aligned}
$$

2. inverse of $\prod_{n-1} \frac{1}{x-Q} \prod_{n-1}$ :

$$
\begin{aligned}
& \left(\Pi_{n-1} \frac{1}{x-Q} \Pi_{n-1}\right) \cdot\left(\Pi_{n-1}\left(1+\frac{\mathbf{e}_{n-1} \hat{\phi}_{\infty}^{t}}{\hat{\phi}_{n-1}} \Pi^{n}\right)(x-Q) \Pi_{n-1}\right) \\
= & \Pi_{n-1}\left(R+\psi_{\infty}{\hat{\phi_{\infty}}}^{t} \Pi_{n-1}\right)\left(1+\frac{\mathbf{e}_{n-1}{\hat{\phi_{\infty}}}^{t}}{\hat{\phi}_{n-1}} \Pi^{n}\right)(x-Q) \Pi_{n-1} \\
= & \Pi_{n-1}\left(R+R \frac{\mathbf{e}_{n-1}{\hat{\phi_{\infty}}}^{t}}{\hat{\phi}_{n-1}} \Pi^{n}+\psi_{\infty} \hat{\phi}_{\infty}^{t} \Pi_{n-1}+\psi_{\infty} \hat{\phi}_{\infty}^{t} \frac{t \mathbf{e}_{n-1} \hat{\phi}_{\infty}^{t}}{\hat{\phi}_{n-1}} \Pi^{n}\right) \\
& \left(x-\hat{\Pi_{n-1}}\right.
\end{aligned}
$$




$$
\begin{aligned}
& =\Pi_{n-1}\left(R+\psi_{\infty}{\hat{\phi_{\infty}}}^{t}\left(\Pi_{n-1}+\Pi^{n}\right)\right)(x-Q) \Pi_{n-1} \\
& =\Pi_{n-1}\left(R+\psi_{\infty}{\hat{\phi_{\infty}}}^{t}\right)(x-Q) \Pi_{n-1} \\
& =\operatorname{Id}_{n}
\end{aligned}
$$

3. inverse of $\Pi_{n-1}(x-Q)\left(y-P^{t}\right) \Pi_{n-1}$ :

$$
\begin{aligned}
& \left(\Pi_{n-1}(x-Q)\left(y-P^{t}\right) \Pi_{n-1}\right) \cdot\left(\Pi_{n-1} \tilde{R}^{t} \Pi_{n}\left(1-\frac{1}{K_{n+1}} \psi_{\infty} \phi_{\infty}^{t}\right) \Pi_{n} R \Pi_{n-1}\right) \\
& =\quad \Pi_{n-1}(x-Q)\left(y-P^{t}\right) \tilde{R}^{t} \Pi_{n}\left(1-\frac{1}{K_{n+1}} \psi_{\infty} \phi_{\infty}^{t}\right) \Pi_{n} R \Pi_{n-1} \\
& =\quad \Pi_{n-1}(x-Q)\left(\Pi_{n}-\frac{\mathbf{e}_{0} \phi_{\infty}^{t}}{\phi_{0}} \Pi_{n}\right)\left(1-\frac{1}{K_{n+1}} \psi_{\infty} \phi_{\infty}^{t}\right) \Pi_{n} R \Pi_{n-1} \\
& =\Pi_{n-1}(x-Q)\left(\Pi_{n}-\Pi_{n} \frac{\psi_{\infty} \phi_{\infty}{ }^{t}}{K_{n+1}}-\frac{\mathbf{e}_{0} \phi_{\infty}{ }^{t}}{\phi_{0}} \Pi_{n}+\frac{\mathbf{e}_{0} \phi_{\infty}{ }^{t}}{\phi_{0}} \Pi_{n} \frac{\psi_{\infty} \phi_{\infty}{ }^{t}}{K_{n+1}}\right) \Pi_{n} R \Pi_{n-1} \\
& =\quad \Pi_{n-1}(x-Q)\left(\Pi_{n}-\Pi_{n} \frac{\psi_{\infty} \phi_{\infty}{ }^{t}}{K_{n+1}}-\frac{\mathbf{e}_{0} \phi_{\infty}{ }^{t}}{\phi_{0}} \Pi_{n}+\frac{\mathbf{e}_{0} \phi_{\infty}{ }^{t}}{\phi_{0}}\right) \Pi_{n} R \Pi_{n-1} \\
& =\quad \Pi_{n-1}(x-Q)\left(\Pi_{n}-\Pi_{n} \frac{\psi_{\infty} \phi_{\infty}{ }^{t}}{K_{n+1}} \Pi_{n}\right) R \Pi_{n-1} \\
& =\quad \Pi_{n-1}(x-Q)\left(1-\frac{\psi_{\infty} \phi_{\infty} t}{K_{n+1}} \Pi_{n}\right) R \Pi_{n-1} \\
& =\quad \Pi_{n-1}(x-Q) R \Pi_{n-1} \\
& =\quad \operatorname{Id}_{n}
\end{aligned}
$$

4. Proof that:

$$
\begin{aligned}
& \Pi_{n-1} \frac{1}{x-Q} \frac{1}{y-P^{t}} \Pi_{n-1} \\
= & \Pi_{n-1} \frac{1}{x-Q} \Pi_{n-1} \frac{1}{y-P^{t}} \Pi_{n-1}+J_{n} \Pi_{n-1} \psi_{\infty} \phi_{\infty}{ }^{t} \Pi_{n-1} \\
= & \Pi_{n-1} \frac{1}{x-Q} \Pi_{n-2} \frac{1}{y-P^{t}} \Pi_{n-1}+J_{n-1} \Pi_{n-1} \psi_{\infty} \phi_{\infty}{ }^{t} \Pi_{n-1} \\
(2-4) &
\end{aligned}
$$

We have:

$$
\begin{aligned}
& \left(\frac{1}{x-Q} \frac{1}{y-P^{t}}\right)_{n m} \\
= & \frac{1}{\sqrt{h_{n} h_{m}}} \int_{\Gamma} q_{m}\left(y^{\prime}\right) \frac{1}{y-y^{\prime}} \frac{1}{x-x^{\prime}} p_{n}\left(x^{\prime}\right) \omega\left(x^{\prime}, y^{\prime}\right) d x^{\prime} d y^{\prime} \\
= & \frac{1}{\sqrt{h_{n} h_{m}}} \int_{\Gamma} \frac{q_{m}(y)-\left(q_{m}(y)-q_{m}\left(y^{\prime}\right)\right)}{y-y^{\prime}} \frac{p_{n}(x)-\left(p_{n}(x)-p_{n}\left(x^{\prime}\right)\right)}{x-x^{\prime}} d \mu\left(x^{\prime}, y^{\prime}\right) \\
= & \frac{1}{\sqrt{h_{n} h_{m}}} \int_{\Gamma} \frac{q_{m}(y)}{y-y^{\prime}} \frac{p_{n}(x)}{x-x^{\prime}} \omega\left(x^{\prime}, y^{\prime}\right) d x^{\prime} d y^{\prime} \\
& -\frac{1}{\sqrt{h_{n} h_{m}}} \int_{\Gamma} \frac{q_{m}(y)-q_{m}\left(y^{\prime}\right)}{y-y^{\prime}} \frac{p_{n}(x)}{x-x^{\prime}} \omega\left(x^{\prime}, y^{\prime}\right) d x^{\prime} d y^{\prime} \\
& -\frac{1}{\sqrt{h_{n} h_{m}}} \int_{\Gamma} \frac{q_{m}(y)}{y-y^{\prime}} \frac{p_{n}(x)-p_{n}\left(x^{\prime}\right)}{x-x^{\prime}} \omega\left(x^{\prime}, y^{\prime}\right) d x^{\prime} d y^{\prime} \\
& +\frac{1}{\sqrt{h_{n} h_{m}}} \int_{\Gamma} \frac{q_{m}(y)-q_{m}\left(y^{\prime}\right)}{y-y_{n}^{\prime}(x)-p_{n}\left(x^{\prime}\right)}-\omega\left(x^{\prime}, y^{\prime}\right) d x^{\prime} d y^{\prime} \\
& =x^{\prime}
\end{aligned}
$$




$$
\begin{aligned}
= & \psi_{n}(x) \phi_{m}(y) J_{0}(x, y) \\
& +\sum_{l=0}^{m-1} \frac{1}{\sqrt{h_{n} h_{m}}} \int_{\Gamma} \tilde{R}_{m l}(y) q_{l}\left(y^{\prime}\right) \frac{p_{n}(x)}{x-x^{\prime}} \omega\left(x^{\prime}, y^{\prime}\right) d x^{\prime} d y^{\prime} \\
& +\sum_{j=0}^{n-1} \frac{1}{\sqrt{h_{n} h_{m}}} \int_{\Gamma} \frac{q_{m}(y)}{y-y^{\prime}} R_{n j}(x) p_{j}\left(x^{\prime}\right) \omega\left(x^{\prime}, y^{\prime}\right) d x^{\prime} d y^{\prime} \\
& +\sum_{j=0}^{n-1} \sum_{l=0}^{m-1} \frac{1}{\sqrt{h_{n} h_{m}}} \int_{\Gamma} \tilde{R}_{m l}(y) R_{n j}(x) q_{l}\left(y^{\prime}\right) p_{j}\left(x^{\prime}\right) \omega\left(x^{\prime}, y^{\prime}\right) d x^{\prime} d y^{\prime} \\
= & \left(J_{0}(x, y) \psi_{\infty}(x) \phi_{\infty}{ }^{t}(y)+\psi_{\infty}(x) \hat{\phi}_{\infty}^{t}(x) \tilde{R}^{t}(y)+\right. \\
& \left.R(x) \hat{\psi_{\infty}}(y) \phi_{\infty}^{t}(y)+R(x) \tilde{R}^{t}(y)\right)_{n m}
\end{aligned}
$$

Thus:

$$
\frac{1}{x-Q} \frac{1}{y-P^{t}}=J_{0} \psi_{\infty} \phi_{\infty}^{t}+\psi_{\infty}{\hat{\phi_{\infty}}}^{t} \tilde{R}^{t}+R \hat{\psi_{\infty}} \phi_{\infty}^{t}+R \tilde{R}^{t}
$$

Now, we take the $\Pi_{n-1}$ projection:

$$
\begin{aligned}
& \Pi_{n-1} \frac{1}{x-Q} \frac{1}{y-P^{t}} \Pi_{n-1}-J_{0} \Pi_{n-1} \psi_{\infty} \phi_{\infty}{ }^{t} \Pi_{n-1} \\
& =\Pi_{n-1} \psi_{\infty}{\hat{\phi_{\infty}}}^{t} \tilde{R}^{t} \Pi_{n-1}+\Pi_{n-1} R \hat{\psi_{\infty}} \phi_{\infty}^{t} \Pi_{n-1}+\Pi_{n-1} R \tilde{R}^{t} \Pi_{n-1} \\
& =\Pi_{n-1} \psi_{\infty} \hat{\phi}_{\infty}^{t} \Pi_{n-1} \tilde{R}^{t} \Pi_{n-1}+\Pi_{n-1} R \Pi_{n-1} \hat{\psi_{\infty}} \phi_{\infty}^{t} \Pi_{n-1}+ \\
& \Pi_{n-1} R \Pi_{n-1} \tilde{R}^{t} \Pi_{n-1} \\
& =\Pi_{n-1}\left(R+\psi_{\infty}{\hat{\phi_{\infty}}}^{t}\right) \Pi_{n-1}\left(\tilde{R}^{t}+{\hat{\psi_{\infty}}}_{\phi_{\infty}^{t}}^{t}\right) \Pi_{n-1} \\
& -\Pi_{n-1} \psi_{\infty} \hat{\phi_{\infty}}{ }^{t} \Pi_{n-1} \hat{\psi_{\infty}} \phi_{\infty}^{t} \Pi_{n-1} \\
& =\left(J_{n}-J_{0}\right) \Pi_{n-1} \psi_{\infty} \phi_{\infty}{ }^{t} \Pi_{n-1} \\
& +\Pi_{n-1}\left(R+\psi_{\infty}{\hat{\phi_{\infty}}}^{t}\right) \Pi_{n-1}\left(\tilde{R}^{t}+{\hat{\psi_{\infty}}}_{\phi_{\infty}^{t}}^{t}\right) \Pi_{n-1} \\
& =\Pi_{n-1} \frac{1}{x-Q} \Pi_{n-1} \frac{1}{y-P^{t}} \Pi_{n-1}+J_{n} \Pi_{n-1} \psi_{\infty} \phi_{\infty}{ }^{t} \Pi_{n-1}
\end{aligned}
$$

Similarly we have:

$$
\begin{aligned}
& \Pi_{n-1} \frac{1}{x-Q} \frac{1}{y-P^{t}} \Pi_{n-1}-J_{0} \Pi_{n-1} \psi_{\infty} \phi_{\infty}^{t} \Pi_{n-1} \\
& =\Pi_{n-1} \psi_{\infty}{\hat{\phi_{\infty}}}^{t} \tilde{R}^{t} \Pi_{n-1}+\Pi_{n-1} R \hat{\psi_{\infty}} \phi_{\infty}^{t} \Pi_{n-1}+\Pi_{n-1} R \tilde{R}^{t} \Pi_{n-1} \\
& =\Pi_{n-1} \psi_{\infty}{\hat{\phi_{\infty}}}^{t} \Pi_{n-2} \tilde{R}^{t} \Pi_{n-1}+\Pi_{n-1} R \Pi_{n-2} \hat{\psi_{\infty}} \phi_{\infty}^{t} \Pi_{n-1}+\Pi_{n-1} R \Pi_{n-2} \tilde{R}^{t} \Pi_{n-1} \\
& =\Pi_{n-1}\left(R+\psi_{\infty}{\hat{\phi_{\infty}}}^{t}\right) \Pi_{n-2}\left(\tilde{R}^{t}+\hat{\psi_{\infty}} \phi_{\infty}^{t}\right) \Pi_{n-1}-\Pi_{n-1} \psi_{\infty}{\hat{\phi_{\infty}}}^{t} \Pi_{n-2} \hat{\psi_{\infty}} \phi_{\infty}^{t} \Pi_{n-1} \\
& =\left(J_{n-1}-J_{0}\right) \Pi_{n-1} \psi_{\infty} \phi_{\infty}^{t} \Pi_{n-1}+\Pi_{n-1}\left(R+\psi_{\infty}{\hat{\phi_{\infty}}}^{t}\right) \Pi_{n-2}\left(\tilde{R}^{t}+\hat{\psi_{\infty}} \phi_{\infty}^{t}\right) \Pi_{n-1} \\
& =\Pi_{n-1} \frac{1}{x-Q} \Pi_{n-2} \frac{1}{y-P^{t}} \Pi_{n-1}+J_{n-1} \Pi_{n-1} \psi_{\infty} \phi_{\infty}{ }^{t} \Pi_{n-1}
\end{aligned}
$$

5. - inverse of $\Pi_{n-1} \frac{1}{x-Q} \frac{1}{y-P^{t}} \Pi_{n-1}$ :

First, notice that:

$$
{\hat{\phi_{\infty}}}^{t} \Pi^{n-1}(x-Q) \Pi_{n-1}={\hat{\phi_{\infty}}}^{t}\left(1-\Pi_{n-2}\right)(x-Q) \Pi_{n-1}
$$




$$
\begin{aligned}
& ={\hat{\phi_{\infty}}}^{t}(x-Q) \Pi_{n-1}-{\hat{\phi_{\infty}}}^{t} \Pi_{n-2}(x-Q) \Pi_{n-1} \\
& =\frac{\hat{\mathbf{e}}_{0}^{t}}{\psi_{0}}-{\hat{\phi_{\infty}}}^{t} \Pi_{n-2}(x-Q)
\end{aligned}
$$

Now, compute:

$$
\begin{aligned}
& \Pi_{n-1}\left(y-P^{t}\right)\left(\Pi_{n-2}+\Pi^{n-1} \frac{\hat{\psi_{\infty}}}{{\hat{\phi_{\infty}}}^{t}} \Pi^{n-1}\right)(x-Q) \Pi_{n-1} \\
= & \Pi_{n-1}\left(y-P^{t}\right)\left(\Pi_{n-2}(x-Q)+\frac{1}{J_{n-1}} \Pi^{n-1} \hat{\psi_{\infty}}\left(\frac{\mathbf{e}_{0}^{t}}{\psi_{0}}-{\hat{\phi_{\infty}}}^{t} \Pi_{n-2}(x-Q)\right)\right) \\
= & \Pi_{n-1}\left(y-P^{t}\right)\left(\left(1-\Pi^{n-1} \frac{\hat{\psi_{\infty}}}{{\hat{\phi_{\infty}}}^{t}}\right) \Pi_{n-2}(x-Q)+\Pi^{n-1} \frac{\hat{\psi_{\infty}} \mathbf{e}_{0}^{t}}{J_{n-1} \psi_{0}}\right)
\end{aligned}
$$

Thus:

$$
\begin{aligned}
& \Pi_{n-1}\left(y-P^{t}\right)\left(\Pi_{n-2}+\Pi^{n-1} \frac{\hat{\psi_{\infty}}{\hat{\phi_{\infty}}}^{t}}{{\hat{J_{n-1}}}_{t}} \Pi^{n-1}\right)(x-Q) \Pi_{n-1} \frac{1}{x-Q} \frac{1}{y-P^{t}} \Pi_{n-1} \\
& =\Pi_{n-1}\left(y-P^{t}\right)\left(1-\Pi^{n-1} \frac{\hat{\hat{\psi}_{\infty}}{\hat{\phi_{\infty}}}^{t}}{J_{n-1}}\right) \Pi_{n-2} \frac{1}{y-P^{t}} \Pi_{n-1} \\
& +\Pi_{n-1}\left(y-P^{t}\right) \Pi^{n-1} \frac{\hat{\psi_{\infty}}}{J_{n-1} \psi_{0}} \mathbf{e}_{0}^{t} \frac{1}{x-Q} \frac{1}{y-P^{t}} \Pi_{n-1} \\
& =\Pi_{n-1}\left(y-P^{t}\right)\left(1-\Pi^{n-1} \frac{\hat{\psi_{\infty}}{\hat{\phi_{\infty}}}^{t}}{J_{n-1}}\right) \Pi_{n-2}\left(\tilde{R}^{t}+\hat{\psi_{\infty}} \phi_{\infty}^{t}\right) \Pi_{n-1} \\
& +\Pi_{n-1}\left(y-P^{t}\right) \Pi^{n-1} \frac{\hat{\psi_{\infty}}}{J_{n-1} \psi_{0}} \mathbf{e}_{0}^{t}\left(R+\psi_{\infty}{\hat{\phi_{\infty}}}^{t}\right) \Pi_{n-2}\left(\tilde{R}^{t}+{\hat{\psi_{\infty}}}_{\phi_{\infty}}^{t}\right) \Pi_{n-1} \\
& +\Pi_{n-1}\left(y-P^{t}\right) \Pi^{n-1} \frac{\hat{\psi_{\infty}}}{J_{n-1} \psi_{0}} \mathbf{e}_{0}^{t}\left(J_{n-1} \psi_{\infty} \phi_{\infty}^{t}\right) \Pi_{n-1} \\
& =\Pi_{n-1}\left(y-P^{t}\right)\left(1-\Pi^{n-1} \frac{\hat{\psi_{\infty}}{\hat{\phi_{\infty}}}^{t}}{J_{n-1}}\right)\left(\tilde{R}^{t}+\Pi_{n-2} \hat{\psi_{\infty}} \phi_{\infty}^{t}\right) \Pi_{n-1} \\
& +\Pi_{n-1}\left(y-P^{t}\right) \Pi^{n-1} \frac{\hat{\psi_{\infty}}}{{J_{n-1}}_{\phi_{\infty}}^{t}}\left(\tilde{R}^{t}+\Pi_{n-2} \hat{\psi_{\infty}} \phi_{\infty}^{t}\right) \Pi_{n-1} \\
& +\Pi_{n-1}\left(y-P^{t}\right) \Pi^{n-1}{\hat{\psi_{\infty}}}_{\infty}^{t} \Pi_{n-1} \\
& =\Pi_{n-1}\left(y-P^{t}\right)\left(\tilde{R}^{t}+\Pi_{n-2} \hat{\psi_{\infty}} \phi_{\infty}^{t}\right) \Pi_{n-1}+\Pi_{n-1}\left(y-P^{t}\right) \Pi^{n-1} \hat{\psi}_{\infty} \phi_{\infty}^{t} \Pi_{n-1} \\
& =\Pi_{n-1}\left(y-P^{t}\right)\left(\tilde{R}^{t}+\hat{\psi_{\infty}} \phi_{\infty}^{t}\right) \Pi_{n-1} \\
& =\operatorname{Id}_{n}
\end{aligned}
$$

\section{B.2 Determinants}

1. - determinant of $\Pi_{n-1}(x-Q) \Pi_{n-1}$. This is a classical result and can be found in the litterature. 
One possible proof, is that $\operatorname{det}\left(\Pi_{n-1}(x-Q) \Pi_{n-1}\right)$ is a monic polynomial of degree $n$. Consider $x$ a zeroe of $p_{n}(x)$, this implies that $\Pi_{n-1} \psi_{\infty}(x)=\Pi_{n} \psi_{\infty}(x)$, thus:

$$
\Pi_{n-1}(x-Q) \Pi_{n-1} \psi_{\infty}(x)=\Pi_{n-1}(x-Q) \Pi_{n} \psi_{\infty}(x)=\Pi_{n-1}(x-Q) \psi_{\infty}(x)=0
$$

Thus, the $n$ zeroes of $\psi_{n}$ are also the zeroes of $\operatorname{det}\left(\Pi_{n-1}(x-Q) \Pi_{n-1}\right)$. The converse is easy too.

Thus we have:

$$
\operatorname{det}\left(\Pi_{n-1}(x-Q) \Pi_{n-1}\right)=p_{n}(x) \quad, \quad \operatorname{det}\left(\Pi_{n-1}(y-P) \Pi_{n-1}\right)=q_{n}(y)
$$

2. - determinant of $\Pi_{n-1} \frac{1}{x-Q} \Pi_{n-1}$ :

Compute:

$$
\begin{aligned}
& p_{n}(x) \operatorname{det}\left(\Pi_{n-1} \frac{1}{x-Q} \Pi_{n-1}\right) \\
= & \operatorname{det}\left(\Pi_{n-1}(x-Q) \Pi_{n-1} \frac{1}{x-Q} \Pi_{n-1}\right) \\
= & \operatorname{det}\left(\Pi_{n-1}(x-Q) \Pi_{n-1}\left(R+\psi_{\infty}{\hat{\phi_{\infty}}}^{t}\right) \Pi_{n-1}\right) \\
= & \operatorname{det}\left(\Pi_{n-1}(x-Q)\left(\Pi_{n}-\mathbf{e}_{n} \mathbf{e}_{n}^{t}\right)\left(R+\psi_{\infty} \hat{\phi}_{\infty}^{t}\right) \Pi_{n-1}\right) \\
= & \left.\operatorname{det}\left(\Pi_{n-1}(x-Q)\left(R+\psi_{\infty} \hat{\phi}_{\infty}^{t}\right) \Pi_{n-1} \hat{R}^{t}\right) \Pi_{n-1}\right) \\
= & \operatorname{det}\left(\operatorname{Id}_{n}+\gamma_{n-1}(x-Q) \mathbf{e}_{n-1} \mathbf{e}_{n}^{t}\left(R+\psi_{\infty}\left(R+\hat{\psi}_{\infty} \hat{\phi}_{\infty}^{t}\right) \Pi_{n-1}\right)\right. \\
= & 1+\gamma_{n} \mathbf{e}_{n}^{t}\left(R+\psi_{\infty} \hat{\phi}_{\infty}^{t}\right) \mathbf{e}_{n-1} \\
= & 1+\gamma_{n}\left(R_{n, n-1}+\psi_{n} \hat{\phi}_{n-1}\right) \\
= & 1+\gamma_{n}\left(-\frac{1}{\gamma_{n}}+\psi_{n} \hat{\phi}_{n-1}\right) \\
= & \gamma_{n} \psi_{n} \hat{\phi}_{n-1}
\end{aligned}
$$

i.e.

$$
\operatorname{det}\left(\Pi_{n-1} \frac{1}{x-Q} \Pi_{n-1}\right)=\frac{\gamma_{n} \mathrm{e}^{-V_{1}}}{\sqrt{h_{n}}} \hat{\phi}_{n-1}=\frac{\mathrm{e}^{-V_{1}}}{\sqrt{h_{n-1}}} \hat{\phi}_{n-1}
$$

3. kernel $K_{n}$ :

$$
\begin{aligned}
& \operatorname{det}\left(\Pi_{n-1}(x-Q)\left(y-P^{t}\right) \Pi_{n-1}\right) \\
= & \operatorname{det}\left(\Pi_{n-1}(x-Q) \Pi_{n-1}\left(y-P^{t}\right) \Pi_{n-1}+\Pi_{n-1}(x-Q) \Pi^{n}\left(y-P^{t}\right) \Pi_{n-1}\right) \\
= & \operatorname{det}\left(\Pi_{n-1}(x-Q) \Pi_{n-1}\left(y-P^{t}\right) \Pi_{n-1}+\gamma_{n}^{2} \mathbf{e}_{n-1} \mathbf{e}_{n-1}^{t}\right) \\
= & p_{n}(x) q_{n}(y) \operatorname{det}\left(\operatorname{Id}_{n}+\right. \\
& \left.\quad+\gamma_{n}^{2} \Pi_{n-1}\left(1-\frac{\psi_{\infty} \mathbf{e}_{n}^{t}}{\psi_{n}}\right) \Pi_{n} R \mathbf{e}_{n-1} \mathbf{e}_{n-1}^{t} \tilde{R}^{t} \Pi_{n}\left(1-\frac{\mathbf{e}_{n} \phi_{\infty}^{t}}{\phi_{n}}\right) \Pi_{n-1}\right) \\
& \quad p_{n}(x) q_{n}(y) \operatorname{det}\left(\operatorname{Id}_{n}+\Pi_{n-1}\left(1-\frac{\psi_{\infty} \mathbf{e}_{n}^{t}}{\psi_{n}}\right) \mathbf{e}_{n} \mathbf{e}_{n}^{t}\left(1-\frac{\mathbf{e}_{n} \phi_{\infty}^{t}}{\phi_{n}}\right) \Pi_{n-1}\right)
\end{aligned}
$$




$$
\begin{aligned}
& =p_{n}(x) q_{n}(y)\left(1+\mathbf{e}_{n}^{t}\left(1-\frac{\mathbf{e}_{n} \phi_{\infty}^{t}}{\phi_{n}}\right) \Pi_{n-1}\left(1-\frac{\psi_{\infty} \mathbf{e}_{n}^{t}}{\psi_{n}}\right) \mathbf{e}_{n}\right) \\
& =p_{n}(x) q_{n}(y)\left(1+\frac{K_{n}}{\psi_{n} \phi_{n}}\right) \\
& =p_{n}(x) q_{n}(y) \frac{K_{n+1}}{\psi_{n} \phi_{n}} \\
& =h_{n} \mathrm{e}^{V_{1}(x)+V_{2}(y)} K_{n+1}
\end{aligned}
$$

4. kernel $J_{n}$ :

$$
\begin{aligned}
& \operatorname{det}\left(\Pi_{n-1} \frac{1}{x-Q} \frac{1}{y-P^{t}} \Pi_{n-1}\right) \\
& =\operatorname{det}\left(J_{n} \Pi_{n-1} \psi_{\infty} \phi_{\infty}^{t} \Pi_{n-1}+\Pi_{n-1} \frac{1}{x-Q} \Pi_{n-1} \frac{1}{y-P^{t}} \Pi_{n-1}\right) \\
& =\operatorname{det}\left(\Pi_{n-1} \frac{1}{x-Q} \Pi_{n-1} \frac{1}{y-P^{t}} \Pi_{n-1}\right) \\
& \left(1+J_{n} \phi_{\infty}^{t}\left(\Pi_{n-1} \frac{1}{x-Q} \Pi_{n-1} \frac{1}{y-P^{t}} \Pi_{n-1}\right)^{-1} \psi_{\infty}\right) \\
& =\frac{\mathrm{e}^{-V_{1}-V_{2}}}{h_{n-1}} \hat{\psi}_{n-1} \hat{\phi}_{n-1} \\
& \left(1+J_{n} \phi_{\infty}^{t}\left(\Pi_{n-1} \frac{1}{y-P^{t}} \Pi_{n-1}\right)^{-1}\left(\Pi_{n-1} \frac{1}{x-Q} \Pi_{n-1}\right)^{-1} \psi_{\infty}\right) \\
& =\frac{\mathrm{e}^{-V_{1}-V_{2}}}{h_{n-1}} \hat{\psi}_{n-1} \hat{\phi}_{n-1}\left(1+J_{n} \phi_{\infty}^{t} \Pi_{n-1}\left(y-P^{t}\right)\right.
\end{aligned}
$$

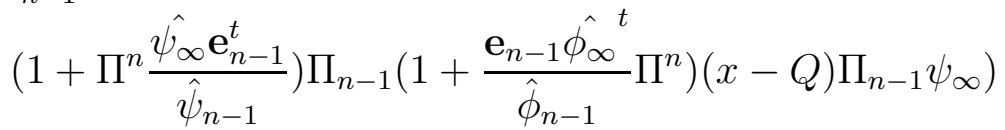

$$
\begin{aligned}
& =\frac{\mathrm{e}^{-V_{1}-V_{2}}}{h_{n-1}} \hat{\psi}_{n-1} \hat{\phi}_{n-1}\left(1+J_{n} \phi_{\infty}^{t} \Pi_{n-1}\left(y-P^{t}\right)\right. \\
& \left.\left(1+\Pi^{n} \frac{\hat{\psi_{\infty}} \mathbf{e}_{n-1}^{t}}{\hat{\psi}_{n-1}}\right) \Pi_{n-1}\left(1+\frac{\mathbf{e}_{n-1} \hat{\phi}_{\infty}^{t}}{\hat{\phi}_{n-1}} \Pi^{n}\right)(x-Q) \Pi_{n-1} \psi_{\infty}\right) \\
& =\frac{\mathrm{e}^{-V_{1}-V_{2}}}{h_{n-1}} \hat{\psi}_{n-1} \hat{\phi}_{n-1}\left(1+J_{n} \phi_{\infty}^{t} \Pi_{n-1}\left(y-P^{t}\right)\right. \\
& \left.\left(1+\Pi^{n} \frac{\hat{\psi_{\infty}} \mathbf{e}_{n-1}^{t}}{\hat{\psi}_{n-1}}\right)\left(\Pi_{n-2}+\mathbf{e}_{n-1} \mathbf{e}_{n-1}^{t}\right)\left(1+\frac{\mathbf{e}_{n-1}{\hat{\phi_{\infty}}}^{t}}{\hat{\phi}_{n-1}} \Pi^{n}\right)(x-Q) \Pi_{n-1} \psi_{\infty}\right) \\
& =\frac{\mathrm{e}^{-V_{1}-V_{2}}}{h_{n-1}} \hat{\psi}_{n-1} \hat{\phi}_{n-1}\left(1+J_{n} \phi_{\infty}^{t} \Pi_{n-1}\left(y-P^{t}\right)\right. \\
& \left.\left(1+\Pi^{n} \frac{\hat{\psi_{\infty}} \mathbf{e}_{n-1}^{t}}{\hat{\psi}_{n-1}}\right) \mathbf{e}_{n-1} \mathbf{e}_{n-1}^{t}\left(1+\frac{\mathbf{e}_{n-1} \hat{\phi}_{\infty}^{t}}{\hat{\phi}_{n-1}} \Pi^{n}\right)(x-Q) \Pi_{n-1} \psi_{\infty}\right) \\
& =\frac{\mathrm{e}^{-V_{1}-V_{2}}}{h_{n-1}} \hat{\psi}_{n-1} \hat{\phi}_{n-1}\left(1+J_{n} \phi_{\infty}^{t} \Pi_{n-1}\left(y-P^{t}\right)\right. \\
& \left.\left(\mathbf{e}_{n-1}+\Pi^{n} \frac{\hat{\psi_{\infty}}}{\hat{\psi}_{n-1}}\right)\left(\mathbf{e}_{n-1}^{t}+\frac{{\hat{\phi_{\infty}}}^{t}}{\hat{\phi}_{n-1}} \Pi^{n}\right)(x-Q) \Pi_{n-1} \psi_{\infty}\right) \\
& =\frac{\mathrm{e}^{-V_{1}-V_{2}}}{h_{n-1}} \hat{\psi}_{n-1} \hat{\phi}_{n-1}(1+
\end{aligned}
$$




$$
\begin{aligned}
& \left.+J_{n} \phi_{\infty}^{t} \Pi_{n-1}\left(y-P^{t}\right) \Pi^{n-1} \frac{\hat{\psi_{\infty}}}{\hat{\psi}_{n-1}} \frac{\hat{\phi}_{\infty}^{t}}{\hat{\phi}_{n-1}} \Pi^{n-1}(x-Q) \Pi_{n-1} \psi_{\infty}\right) \\
= & \frac{\mathrm{e}^{-V_{1}-V_{2}}}{h_{n-1}} \hat{\psi}_{n-1} \hat{\phi}_{n-1}\left(1+J_{n} \phi_{\infty}^{t} \Pi_{n-1}\left(y-P^{t}\right) \frac{\hat{\psi_{\infty}}}{\hat{\psi}_{n-1}} \frac{\hat{\phi}_{\infty}}{\hat{\phi}_{n-1}}(x-Q) \Pi_{n-1} \psi_{\infty}\right) \\
= & \frac{\mathrm{e}^{-V_{1}-V_{2}}}{h_{n-1}} \hat{\psi}_{n-1} \hat{\phi}_{n-1}\left(1+J_{n} \phi_{\infty}^{t} \frac{\mathbf{e}_{0}}{\hat{\psi}_{n-1} \phi_{0}} \frac{\mathbf{e}_{0}^{t}}{\hat{\phi}_{n-1} \psi_{0}} \psi_{\infty}\right) \\
= & \frac{\mathrm{e}^{-V_{1}-V_{2}}}{h_{n-1}} \hat{\psi}_{n-1} \hat{\phi}_{n-1}\left(1+\frac{J_{n}}{\hat{\psi}_{n-1} \hat{\phi}_{n-1}}\right) \\
= & \frac{\mathrm{e}^{-V_{1}-V_{2}}}{h_{n-1}} J_{n-1}
\end{aligned}
$$

\section{Appendix C Matrices $U$ and $\tilde{U}$}

\section{C.3 Definitions}

The following matrices play an important role.

Define:

$$
U_{n}(x, y):=\Pi_{n}\left(y-P^{t}\right) R(x) \Pi^{n-1} \quad, \quad \tilde{U}_{n}(x, y):=\Pi_{n}\left(x-Q^{t}\right) \tilde{R}(y) \Pi^{n-1}
$$

Notice that since $R$ is lower triangular and $P$ is finite band, $U_{n}(x, y)\left(\operatorname{resp} . \tilde{U}_{n}(x, y)\right)$ is a small lower triangular matrix of size $d_{1}+1$ (resp. $\left.d_{2}+1\right)$ :

$$
U_{n}(x, y)=\Pi_{n}^{n-d_{1}}\left(y-P^{t}\right) R(x) \Pi_{n+d_{1}-1}^{n-1}
$$

$U_{n}(x, y)$ (resp. $\left.\tilde{U}_{n}(x, y)\right)$ is linear in $y$ (resp. $x$ ) and of degree at most $d_{1}$ in $x$ (resp. $d_{2}$ in $y$ ).

$U_{n}(x, y)$ can also be rewritten as:

$$
U_{n}(x, y)=-\frac{y+V_{1}^{\prime}(x)}{\gamma_{n}} \mathbf{e}_{n} \mathbf{e}_{n-1}^{t}-\Pi_{n} \frac{V_{1}^{\prime}(x)-V_{1}^{\prime}(Q)}{x-Q} \Pi^{n-1}
$$

proof:

$$
\begin{aligned}
U_{n}(x, y)= & \Pi_{n}^{n-d_{1}}\left(y-P^{t}\right) R(x) \Pi_{n+d_{1}-1}^{n-1} \\
= & \Pi_{n}^{n-d_{1}}\left(y+V_{1}^{\prime}(x)-V_{1}^{\prime}(x)+V_{1}^{\prime}(Q)-V_{1}^{\prime}(Q)-P^{t}\right) R(x) \Pi_{n+d_{1}-1}^{n-1} \\
= & \Pi_{n}^{n-d_{1}}\left(y+V_{1}^{\prime}(x)\right) R(x) \Pi_{n+d_{1}-1}^{n-1}-\Pi_{n}^{n-d_{1}}\left(V_{1}^{\prime}(x)-V_{1}^{\prime}(Q)\right) R(x) \Pi_{n+d_{1}-1}^{n-1} \\
& \quad-\Pi_{n}^{n-d_{1}}\left(V_{1}^{\prime}(Q)+P^{t}\right) R(x) \Pi_{n+d_{1}-1}^{n-1} \\
= & \Pi_{n}^{n-d_{1}}\left(y+V_{1}^{\prime}(x)\right) R(x) \Pi_{n+d_{1}-1}^{n-1}-\Pi_{n}^{n-d_{1}}\left(V_{1}^{\prime}(x)-V_{1}^{\prime}(Q)\right) R(x) \Pi_{n+d_{1}-1}^{n-1} \\
= & \left(y+V_{1}^{\prime}(x)\right) \Pi_{n}^{n-d_{1}} R(x) \Pi_{n+d_{1}-1}^{n-1} \\
& \quad-\Pi_{n}^{n-d_{1}} \frac{V_{1}^{\prime}(x)-V_{1}^{\prime}(Q)}{x-Q}(x-Q) R(x) \Pi_{n+d_{1}-1}^{n-1}
\end{aligned}
$$




$$
\begin{aligned}
& =-\frac{y+V_{1}^{\prime}(x)}{\gamma_{n}} \mathbf{e}_{n} \mathbf{e}_{n-1}^{t}-\Pi_{n}^{n-d_{1}} \frac{V_{1}^{\prime}(x)-V_{1}^{\prime}(Q)}{x-Q} \Pi_{n+d_{1}-1}^{n-1} \\
& =-\frac{y+V_{1}^{\prime}(x)}{\gamma_{n}} \mathbf{e}_{n} \mathbf{e}_{n-1}^{t}-\Pi_{n} \frac{V_{1}^{\prime}(x)-V_{1}^{\prime}(Q)}{x-Q} \Pi^{n-1}
\end{aligned}
$$

Notice also that if $n>d_{2} d_{1}$, we have:

$$
U_{n}(x, y):=\Pi_{n} R(x)\left(y-P^{t}\right) \Pi^{n-1}
$$

\section{C.4 Some properties}

- Multiplication by the Christoffel-Darboux matrix:

$$
\begin{gathered}
-U_{n}(x, y) A_{n}=B_{n+1}^{t}+\left(y-P^{t}\right) \mathbf{e}_{n} \mathbf{e}_{n}^{t}-\Pi_{n}\left(y-P^{t}\right) R \Pi^{n}(x-Q) \\
-A_{n} U_{n}(x, y)=B_{n-1}^{t}+\mathbf{e}_{n-1} \mathbf{e}_{n-1}^{t}\left(y-P^{t}\right)-(x-Q) \Pi_{n-1}\left(y-P^{t}\right) R \Pi^{n-1}
\end{gathered}
$$

and subsequentely:

$$
\begin{aligned}
& \Phi_{\infty}^{t} U_{n}(x, y) A_{n} \Psi_{\infty}=-\Phi_{\infty}^{t} B_{n+1}^{t} \Psi_{\infty} \\
& \hat{\Phi}_{\infty}^{t} A_{n} U_{n}(x, y) \hat{\Psi}_{\infty}=-\hat{\Phi}_{\infty}^{t} B_{n-1}^{t} \hat{\Psi}_{\infty}
\end{aligned}
$$

proof:

$$
\begin{array}{lll} 
& \quad-U_{n}(x, y) A_{n} \\
= & \Pi_{n}\left(y-P^{t}\right) R(x-Q) \Pi^{n}-\Pi_{n}\left(y-P^{t}\right) R \Pi^{n}(x-Q) \\
= & \Pi_{n}\left(y-P^{t}\right) \Pi^{n}-\Pi_{n}\left(y-P^{t}\right) R \Pi^{n}(x-Q) \\
= & {\left[\Pi_{n}, y-P^{t}\right] \Pi^{n}+\left(y-P^{t}\right) \mathbf{e}_{n} \mathbf{e}_{n}^{t}-\Pi_{n}\left(y-P^{t}\right) R \Pi^{n}(x-Q)} \\
= & B_{n+1}^{t}+\left(y-P^{t}\right) \mathbf{e}_{n} \mathbf{e}_{n}^{t}-\Pi_{n}\left(y-P^{t}\right) R \Pi^{n}(x-Q) \\
(3-10) & \\
& \\
& \\
& -A_{n} U_{n}(x, y) \\
= & \Pi_{n-1}(x-Q)\left(y-P^{t}\right) R \Pi^{n-1}-(x-Q) \Pi_{n-1}\left(y-P^{t}\right) R \Pi^{n-1} \\
= & \Pi_{n-1}\left(1+\left(y-P^{t}\right)(x-Q)\right) R \Pi^{n-1}-(x-Q) \Pi_{n-1}\left(y-P^{t}\right) R \Pi^{n-1} \\
= & \Pi_{n-1}\left(y-P^{t}\right) \Pi^{n-1}+\Pi_{n-1} R \Pi^{n-1}-(x-Q) \Pi_{n-1}\left(y-P^{t}\right) R \Pi^{n-1} \\
= & \Pi_{n-1}\left(y-P^{t}\right) \Pi^{n-1}-(x-Q) \Pi_{n-1}\left(y-P^{t}\right) R \Pi^{n-1} \\
= & \Pi_{n-1}\left[y-P^{t}, \Pi^{n-1}\right]+\Pi_{n-1} \Pi^{n-1}\left(y-P^{t}\right) \\
& \quad-(x-Q) \Pi_{n-1}\left(y-P^{t}\right) R \Pi^{n-1}
\end{array}
$$




$$
\begin{array}{ll}
=\quad \Pi_{n-1}\left[P^{t}, \Pi_{n-2}\right]+\mathbf{e}_{n-1} \mathbf{e}_{n-1}^{t}\left(y-P^{t}\right)-(x-Q) \Pi_{n-1}\left(y-P^{t}\right) R \Pi^{n-1} \\
=\quad B_{n-1}^{t}+\mathbf{e}_{n-1} \mathbf{e}_{n-1}^{t}\left(y-P^{t}\right)-(x-Q) \Pi_{n-1}\left(y-P^{t}\right) R \Pi^{n-1}
\end{array}
$$

$(3-11)$

- Inverse:

$$
\tilde{U}_{n}^{t-1}(x, y)=\Pi_{n}^{n-d_{2}}\left(y-P^{t}\right) L(x) \Pi_{n+d_{2}-1}^{n-1}
$$

proof:

$$
\begin{aligned}
& \tilde{U}_{n}^{t}(x, y) \Pi_{n}^{n-d_{2}}\left(y-P^{t}\right) L(x) \Pi_{n+d_{2}-1}^{n-1} \\
= & \Pi^{n-1} \tilde{R}^{t}(y)(x-Q) \Pi_{n}^{n-d_{2}}\left(y-P^{t}\right) L(x) \Pi_{n+d_{2}-1}^{n-1} \\
= & \Pi^{n-1} \tilde{R}^{t}(y) \Pi^{n}(x-Q) \Pi_{n}\left(y-P^{t}\right) \Pi_{n-1} L(x) \Pi_{n+d_{2}-1}^{n-1} \\
= & \Pi^{n-1} \tilde{R}^{t}(y) \Pi^{n}(x-Q)\left(y-P^{t}\right) \Pi_{n-1} L(x) \Pi_{n+d_{2}-1}^{n-1} \\
= & \Pi^{n-1} \tilde{R}^{t}(y) \Pi^{n}\left(y-P^{t}\right)(x-Q) \Pi_{n-1} L(x) \Pi_{n+d_{2}-1}^{n-1} \\
= & \Pi^{n-1} \tilde{R}^{t}(y)\left(y-P^{t}\right)(x-Q) L(x) \Pi_{n+d_{2}-1}^{n-1} \\
= & \Pi_{n+d_{2}-1}^{n-1}
\end{aligned}
$$

- Multiplication of the inverse by the Christoffel-Darboux matrix:

$$
\begin{aligned}
& -\tilde{U}_{n}^{t-1}(x, y) A_{n}=B_{n-d_{2}}^{t} \Pi^{n}+\left(y-P^{t}\right) \mathbf{e}_{n} \mathbf{e}_{n}^{t}-\Pi_{n}^{n-d_{2}}\left(y-P^{t}\right) L(x) \Pi^{n}(x-Q) \\
& A_{n} \tilde{U}_{n}^{t-1}(x, y)=-\Pi^{n} B_{n+d_{2}}^{t}+\Pi_{n+d_{2}-1}^{n}\left(y-P^{t}\right)-(x-Q) \Pi^{n}\left(y-P^{t}\right) L(x) \Pi_{n+d_{2}-1}^{n-1}
\end{aligned}
$$

In particular:

$$
\Phi_{\infty}^{t} \tilde{U}_{n}^{t-1}(x, y) A_{n} \Psi_{\infty}=-\Phi_{\infty}^{t} B_{n-d_{2}}^{t} \Pi_{n-1} \Psi_{\infty}
$$

and

$$
\hat{\Phi}_{\infty}^{t} A_{n} \tilde{U}_{n}^{t-1}(x, y) \hat{\vec{\psi}}=-\hat{\Phi}_{\infty}^{t} \Pi^{n} B_{n+d_{2}}^{t} \hat{\vec{\psi}}
$$

proof:

$$
\begin{array}{ll} 
& \tilde{U}_{n}^{t-1}(x, y) A_{n} \\
= & \Pi_{n}^{n-d_{2}}\left(y-P^{t}\right) L(x)\left[x-Q, \Pi_{n-1}\right] \\
= & \Pi_{n}^{n-d_{2}}\left(y-P^{t}\right) L(x)(x-Q) \Pi_{n-1}-\Pi_{n}^{n-d_{2}}\left(y-P^{t}\right) L(x) \Pi_{n-1}(x-Q) \\
= & \Pi_{n}^{n-d_{2}}\left(y-P^{t}\right) \Pi_{n-1}+\gamma_{n-d_{2}} L_{n-d_{2}-1, n-1} \mathbf{e}_{n-d_{2}} \mathbf{e}_{n-1}^{t}(x-Q)
\end{array}
$$




$$
\begin{array}{ll} 
& -\tilde{U}_{n}^{t-1}(x, y) A_{n} \\
= & \Pi_{n}^{n-d_{2}}\left(y-P^{t}\right) L(x)\left[x-Q, \Pi^{n}\right] \\
= & \Pi_{n}^{n-d_{2}}\left(y-P^{t}\right) L(x)(x-Q) \Pi^{n}-\Pi_{n}^{n-d_{2}}\left(y-P^{t}\right) L(x) \Pi^{n}(x-Q) \\
= & \Pi_{n}^{n-d_{2}}\left(y-P^{t}\right) \Pi^{n}-\Pi_{n}^{n-d_{2}}\left(y-P^{t}\right) L(x) \Pi^{n}(x-Q) \\
= & {\left[\Pi_{n}^{n-d_{2}}, y-P^{t}\right] \Pi^{n}+\left(y-P^{t}\right) \Pi_{n}^{n-d_{2}} \Pi^{n}} \\
& \quad-\Pi_{n}^{n-d_{2}}\left(y-P^{t}\right) L(x) \Pi^{n}(x-Q) \\
= & B_{n-d_{2}}^{t} \Pi^{n}+\left(y-P^{t}\right) \mathbf{e}_{n} \mathbf{e}_{n}^{t}-\Pi_{n}^{n-d_{2}}\left(y-P^{t}\right) L(x) \Pi^{n}(x-Q)
\end{array}
$$

Then:

$$
\begin{array}{ll} 
& A_{n} \tilde{U}_{n}^{t-1}(x, y) \\
= & -\left[x-Q, \Pi^{n}\right]\left(y-P^{t}\right) L(x) \Pi_{n+d_{2}-1}^{n-1} \\
= & \Pi^{n}(x-Q)\left(y-P^{t}\right) L(x) \Pi_{n+d_{2}-1}^{n-1}-(x-Q) \Pi^{n}\left(y-P^{t}\right) L(x) \Pi_{n+d_{2}-1}^{n-1} \\
= & \Pi^{n}\left(1+\left(y-P^{t}\right)(x-Q)\right) L(x) \Pi_{n+d_{2}-1}^{n-1} \\
& \quad-(x-Q) \Pi^{n}\left(y-P^{t}\right) L(x) \Pi_{n+d_{2}-1}^{n-1} \\
= & \Pi^{n}\left(y-P^{t}\right) \Pi_{n+d_{2}-1}^{n-1}+\Pi^{n} L(x) \Pi_{n+d_{2}-1}^{n-1} \\
& \quad-(x-Q) \Pi^{n}\left(y-P^{t}\right) L(x) \Pi_{n+d_{2}-1}^{n-1} \\
= & \Pi^{n}\left(y-P^{t}\right) \Pi_{n+d_{2}-1}-(x-Q) \Pi^{n}\left(y-P^{t}\right) L(x) \Pi_{n+d_{2}-1}^{n-1} \\
= & \Pi^{n}\left[\left(y-P^{t}\right), \Pi_{n+d_{2}-1}\right]+\Pi_{n+d_{2}-1}^{n}\left(y-P^{t}\right) \\
& \quad-(x-Q) \Pi^{n}\left(y-P^{t}\right) L(x) \Pi_{n+d_{2}-1}^{n-1} \\
= & -\Pi^{n} B_{n+d_{2}}^{t}+\Pi_{n+d_{2}-1}^{n}\left(y-P^{t}\right)-(x-Q) \Pi^{n}\left(y-P^{t}\right) L(x) \Pi_{n+d_{2}-1}^{n-1}
\end{array}
$$

$(3-20)$

\section{Appendix D Proof of theorem 7.1}

\section{proof:}

Let us introduce the following $n \times n$ matrices:

$$
\begin{gathered}
\zeta_{n}:=\Pi_{n-1}(x-Q)\left(y-P^{t}\right) \Pi_{n-1} \\
\xi_{n}:=\Pi_{n-1} \frac{1}{x-Q} \frac{1}{y-P^{t}} \Pi_{n-1} \\
\omega_{n}:=1_{n}+\Pi_{n-1}\left(y-P^{t}\right) \Pi_{n-2}(x-Q) \Pi_{n-1}
\end{gathered}
$$


and, according to formulae of section 5.1

$$
\begin{gathered}
\operatorname{det} \zeta_{n}=h_{n} K_{n+1} \quad, \quad \zeta_{n}^{-1}=\Pi_{n-1} \tilde{R}^{t} \Pi_{n}\left(1-\frac{1}{K_{n+1}} \psi_{\infty} \phi_{\infty}^{t}\right) \Pi_{n} R \Pi_{n-1} \\
\operatorname{det} \xi_{n}=\frac{J_{n-1}}{h_{n-1}} \\
\xi_{n}^{-1}=\Pi_{n-1}\left(y-P^{t}\right)\left(\Pi_{n-2}+\frac{1}{J_{n-1}} \Pi^{n-1} \hat{\hat{\psi}_{\infty}}{\hat{\phi_{\infty}}}^{t} \Pi^{n-1}\right)(x-Q) \Pi_{n-1}
\end{gathered}
$$

We have:

$$
\begin{array}{rll}
\omega_{n} & =1_{n}+\Pi_{n-1}\left(y-P^{t}\right) \Pi_{n-2}(x-Q) \Pi_{n-1} \\
& =1_{n}+\Pi_{n-1}\left(y-P^{t}\right)(x-Q) \Pi_{n-1}-\Pi_{n-1}\left(y-P^{t}\right) \Pi^{n-1}(x-Q) \Pi_{n-1} \\
& =\quad \zeta_{n}-\Pi_{n-1}\left(y-P^{t}\right) \Pi^{n-1}(x-Q) \Pi_{n-1} \\
& = & \zeta_{n}\left(1-\frac{1}{\zeta_{n}} \Pi_{n-1}\left(y-P^{t}\right) \Pi^{n-1}(x-Q) \Pi_{n-1}\right) \\
(4-7) &
\end{array}
$$

Thus:

$$
\begin{array}{lll} 
& \quad \operatorname{det} \omega_{n} \\
= & \operatorname{det} \zeta_{n} \operatorname{det}\left(1-\frac{1}{\zeta_{n}} \Pi_{n-1}\left(y-P^{t}\right) \Pi^{n-1}(x-Q) \Pi_{n-1}\right) \\
= & h_{n} K_{n+1} \operatorname{det}\left(1-\Pi_{n-1} \tilde{R}^{t} \Pi_{n}\left(1-\frac{1}{K_{n+1}} \psi_{\infty} \phi_{\infty}^{t}\right) \Pi_{n} R \times\right. \\
& \left.\quad \times\left(y-P^{t}\right) \Pi^{n-1}(x-Q) \Pi_{n-1}\right) \\
= & h_{n} K_{n+1} \operatorname{det}\left(1-\Pi_{n-1} \tilde{R}^{t} \Pi_{n}\left(1-\frac{1}{K_{n+1}} \psi_{\infty} \phi_{\infty}^{t}\right) U_{n}(x-Q) \Pi_{n-1}\right) \\
= & h_{n} K_{n+1} \operatorname{det}\left(1-\Pi_{n}\left(1-\frac{1}{K_{n+1}} \psi_{\infty} \phi_{\infty}^{t}\right) U_{n} \tilde{U}_{n}^{t}\right) \\
(4-8) &
\end{array}
$$

and:

$$
\begin{aligned}
\operatorname{det} \omega_{n} & =h_{n} K_{n+1} \operatorname{det}\left(1-U_{n} \tilde{U}_{n}^{t}+\frac{1}{K_{n+1}} \Pi_{n} \psi_{\infty} \phi_{\infty}{ }^{t} U_{n} \tilde{U}_{n}^{t}\right) \\
& =h_{n} K_{n+1} \operatorname{det}\left(1-U_{n} \tilde{U}_{n}^{t}\right)\left(1+\frac{1}{K_{n+1}} \phi_{\infty}{ }^{t} U_{n} \tilde{U}_{n}^{t} \frac{1}{1-U_{n} \tilde{U}_{n}^{t}} \psi_{\infty}\right) \\
& =h_{n} \operatorname{det}\left(1-U_{n} \tilde{U}_{n}^{t}\right)\left(\phi_{\infty}{ }^{t} \frac{1}{1-U_{n} \tilde{U}_{n}^{t}} \psi_{\infty}\right)
\end{aligned}
$$$$
(4-9)
$$

Inverse:

$$
\omega_{n}^{-1}
$$




$$
\begin{array}{ccc}
= & \left(1-\frac{1}{\zeta_{n}} \Pi_{n-1}\left(y-P^{t}\right) \Pi^{n-1}(x-Q) \Pi_{n-1}\right)^{-1} \zeta_{n}^{-1} \\
= & \left(1-\Pi_{n-1} \tilde{R}^{t} \Pi_{n}\left(1-\frac{\psi_{\infty} \phi_{\infty}^{t}}{K_{n+1}}\right) U_{n}(x-Q) \Pi_{n-1}\right)^{-1} \zeta_{n}^{-1} \\
= & \left(1+\Pi_{n-1} \tilde{R}^{t} \Pi_{n} \frac{1}{1-\Pi_{n}\left(1-\frac{\psi_{\infty} \phi_{\infty}^{t}}{K_{n+1}}\right) U_{n} \tilde{U}_{n}^{t}} \Pi_{n}\left(1-\frac{\psi_{\infty} \phi_{\infty}{ }^{t}}{K_{n+1}}\right) U_{n} \times\right. \\
& \left.\quad(x-Q) \Pi_{n-1}\right) \zeta_{n}^{-1} \\
= & \zeta_{n}^{-1}+\Pi_{n-1} \tilde{R}^{t} \Pi_{n} \frac{1}{1-\Pi_{n}\left(1-\frac{\psi_{\infty} \phi_{\infty}^{t}}{K_{n+1}}\right) U_{n} \tilde{U}_{n}^{t}} \Pi_{n}\left(1-\frac{\psi_{\infty} \phi_{\infty}{ }^{t}}{K_{n+1}}\right) U_{n} \times \\
& \times \tilde{U}_{n}^{t}\left(1-\frac{\psi_{\infty} \phi_{\infty}^{t}}{K_{n+1}}\right) \Pi_{n} R \Pi_{n-1} \\
& \Pi_{n-1} \tilde{R}^{t} \Pi_{n} \frac{1}{1-\Pi_{n}\left(1-\frac{\psi_{\infty} \phi_{\infty}^{t}}{K_{n+1}}\right) U_{n} \tilde{U}_{n}^{t}}\left(1-\frac{\psi_{\infty} \phi_{\infty}^{t}}{K_{n+1}}\right) \Pi_{n} R \Pi_{n-1}
\end{array}
$$

$(4-10)$

Now, the formula of [8] gives $W_{n}=\operatorname{det}\left(1+\xi_{n}\right)$, thus we compute:

$$
\begin{array}{ll} 
& W_{n} \\
= & \operatorname{det}\left(1+\xi_{n}\right) \\
= & \operatorname{det} \xi_{n} \operatorname{det}\left(1+\xi_{n}^{-1}\right) \\
= & \operatorname{det} \xi_{n} \operatorname{det}\left(1+\Pi_{n-1}\left(y-P^{t}\right)\left(\Pi_{n-2}+\frac{1}{J_{n-1}} \Pi^{n-1} \hat{\psi_{\infty}}{\hat{\phi_{\infty}}}^{t} \Pi^{n-1}\right)(x-Q) \Pi_{n-1}\right) \\
= & \operatorname{det} \xi_{n} \operatorname{det}\left(\omega_{n}+\frac{1}{J_{n-1}} \Pi_{n-1}\left(y-P^{t}\right) \Pi^{n-1} \hat{\psi_{\infty}}{ }^{n-1}(x-Q) \Pi_{n-1}\right) \\
= & \operatorname{det} \xi_{n} \operatorname{det} \omega_{n}\left(1+\frac{1}{J_{n-1}} \hat{\phi}_{\infty}^{n-1}(x-Q) \Pi_{n-1} \omega_{n}^{-1} \Pi_{n-1}\left(y-P^{t}\right) \Pi^{n-1} \hat{\psi_{\infty}}\right)
\end{array}
$$

Thus:

$$
\begin{array}{ll} 
& h_{n-1} W_{n} \\
= & \operatorname{det} \omega_{n}\left(J_{n-1}+{\hat{\phi_{\infty}}}^{t} \Pi^{n-1}(x-Q) \Pi_{n-1} \omega_{n}^{-1} \Pi_{n-1}\left(y-P^{t}\right) \Pi^{n-1} \hat{\psi_{\infty}}\right) \\
= & \operatorname{det} \omega_{n}\left(J_{n-1}+{\hat{\phi_{\infty}}}^{t} \tilde{U}_{n}^{t} \frac{1}{1-\Pi_{n}\left(1-\frac{\psi_{\infty} \phi_{\infty} t}{K_{n+1}}\right) U_{n} \tilde{U}_{n}^{t}}\left(1-\frac{\psi_{\infty} \phi_{\infty}}{K_{n+1}}\right) U_{n} \hat{\psi_{\infty}}\right)
\end{array}
$$

i.e.

$$
W_{n}=\gamma_{n}^{2} K_{n+1} J_{n-1} \operatorname{det}\left(1-\Pi_{n}\left(1-\frac{\psi_{\infty} \phi_{\infty}^{t}}{K_{n+1}}\right) U_{n}\left(1-\frac{\hat{\psi_{\infty}}}{{\hat{\phi_{\infty}}}^{t}}\right){\tilde{U_{n-1}^{t}}}_{n}\right)
$$

The two other formulae are obtained by Weinstein-Aronstein duality.

The lemma 1.2 gives Eq. (7-7). 


\section{Appendix E Proof of theorem 7.3}

The formula of 8 gives:

$$
W_{n}(x, y)=\operatorname{det}_{n}\left(\operatorname{Id}_{n}+\pi_{n-1} \frac{1}{x-Q} \frac{1}{y-P^{t}} \pi_{n-1}^{t}\right)
$$

Using Eq. (5-10), it can be rewritten as:

$$
\begin{aligned}
& W_{n}(x, y)=\operatorname{det}_{n}\left(\operatorname{Id}_{n}+J_{n}(x, y) \pi_{n-1} \psi_{\infty} \phi_{\infty}{ }^{t} \pi_{n-1}^{t}+\pi_{n-1} \frac{1}{x-Q} \Pi_{n-1} \frac{1}{y-P^{t}} \pi_{n-1}^{t}\right) \\
& W_{n+1}(x, y)=\operatorname{det}_{n+1}\left(\operatorname{Id}_{n+1}+J_{n}(x, y) \pi_{n} \psi_{\infty} \phi_{\infty}{ }^{t} \pi_{n}^{t}+\pi_{n} \frac{1}{x-Q} \Pi_{n-1} \frac{1}{y-P^{t}} \pi_{n}^{t}\right)
\end{aligned}
$$

The difference is thus the same determinant with substracting 1 in the $n, n$ position:

$$
\begin{aligned}
& W_{n+1}(x, y)-W_{n}(x, y) \\
= & \operatorname{det}_{n+1}\left(\Pi_{n-1}+J_{n} \pi_{n} \psi_{\infty} \phi_{\infty}{ }^{t} \pi_{n}^{t}+\pi_{n} \frac{1}{x-Q} \Pi_{n-1} \frac{1}{y-P^{t}} \pi_{n}^{t}\right)
\end{aligned}
$$

For any arbitrary non-vanishing $\alpha$ and $\tilde{\alpha}$, multiply the matrix inside the determinant

Eq. (5-4) on the left by $\operatorname{Id}_{n+1}-\pi_{n} \frac{\psi_{\infty} \mathbf{e}_{n}^{t}}{\psi_{n}}+\alpha \frac{\mathbf{e}_{n} \mathbf{e}_{n}^{t}}{\psi_{n}}$ (whose determinant is $\frac{\alpha}{\psi_{n}}$ ), and on the right by $\operatorname{Id}_{n+1}-\frac{\mathbf{e}_{n} \phi_{\infty}^{t}}{\phi_{n}} \pi_{n}^{t}+\tilde{\alpha} \frac{\mathbf{e}_{n} \mathbf{e}_{n}^{t}}{\phi_{n}}$ (whose determinant is $\frac{\tilde{\alpha}}{\phi_{n}}$ ):

$$
\begin{gathered}
\left(\operatorname{Id}_{n+1}-\pi_{n} \frac{\psi_{\infty} \mathbf{e}_{n}^{t}}{\psi_{n}}+\alpha \frac{\mathbf{e}_{n} \mathbf{e}_{n}^{t}}{\psi_{n}}\right) \Pi_{n-1}\left(\operatorname{Id}_{n+1}-\frac{\mathbf{e}_{n} \phi_{\infty}^{t}}{\phi_{n}} \pi_{n}^{t}+\tilde{\alpha} \frac{\mathbf{e}_{n} \mathbf{e}_{n}^{t}}{\phi_{n}}\right)=\Pi_{n-1}=\operatorname{Id}_{n+1}-\mathbf{e}_{n} \mathbf{e}_{n}^{t} \\
\left(\operatorname{Id}_{n+1}-\pi_{n} \frac{\psi_{\infty} \mathbf{e}_{n}^{t}}{\psi_{n}}+\alpha \frac{\mathbf{e}_{n} \mathbf{e}_{n}^{t}}{\psi_{n}}\right) J_{n} \psi_{\infty} \phi_{\infty}{ }^{t}\left(\operatorname{Id}_{n+1}-\frac{\mathbf{e}_{n} \phi_{\infty}{ }^{t}}{\phi_{n}} \pi_{n}^{t}+\tilde{\alpha} \frac{\mathbf{e}_{n} \mathbf{e}_{n}^{t}}{\phi_{n}}\right)=J_{n} \alpha \tilde{\alpha} \mathbf{e}_{n} \mathbf{e}_{n}^{t} \text { (5-6) } \\
\quad\left(\operatorname{Id}_{n+1}-\pi_{n} \frac{\psi_{\infty} \mathbf{e}_{n}^{t}}{\psi_{n}}+\alpha \frac{\mathbf{e}_{n} \mathbf{e}_{n}^{t}}{\psi_{n}}\right) \pi_{n} \frac{1}{x-Q} \Pi_{n-1} \\
=\quad\left(\Pi_{n-1}\left(1-\frac{\psi_{\infty} \mathbf{e}_{n}^{t}}{\psi_{n}}\right)+\alpha \frac{\mathbf{e}_{n} \mathbf{e}_{n}^{t}}{\psi_{n}}\right)\left(R+\psi_{\infty} \hat{\phi}_{\infty}^{t}\right) \Pi_{n-1} \\
=\quad \Pi_{n-1}\left(1-\frac{\psi_{\infty} \mathbf{e}_{n}^{t}}{\psi_{n}}\right) \Pi_{n} R \Pi_{n-1}+\alpha \frac{\mathbf{e}_{n} \mathbf{e}_{n}^{t} R+\alpha \mathbf{e}_{n} \hat{\phi}_{\infty}{ }^{t} \Pi_{n-1}}{\psi_{n}} \\
=\quad \Pi_{n-1}\left(1-\frac{\psi_{\infty} \mathbf{e}_{n}^{t}}{\psi_{n}}\right) \Pi_{n} R \Pi_{n-1}+\alpha \mathbf{e}_{n} T_{n}^{t} \\
(5-7)
\end{gathered}
$$

where

$$
T_{n}^{t}=\frac{\mathbf{e}_{n}^{t} R}{\psi_{n}}+\hat{\phi}_{\infty}^{t} \Pi_{n-1} \quad, \quad \tilde{T}_{n}=\frac{\tilde{R}^{t} \mathbf{e}_{n}}{\phi_{n}}+\Pi_{n-1} \hat{\psi_{\infty}}
$$


Finaly, using Eq. (5-6) we have:

$$
\begin{array}{ll} 
& \frac{\alpha \tilde{\alpha}}{\psi_{n} \phi_{n}}\left(W_{n+1}(x, y)-W_{n}(x, y)\right) \\
=\quad & \operatorname{det}_{n+1}\left(\operatorname{Id}_{n+1}+\left(J_{n} \alpha \tilde{\alpha}-1\right) \mathbf{e}_{n} \mathbf{e}_{n}^{t}+\right. \\
& \left.+\left(\left(\Pi_{n-1}(x-Q) \Pi_{n-1}\right)^{-1}+\alpha \mathbf{e}_{n} T_{n}^{t}\right)\left(\left(\Pi_{n-1}\left(y-P^{t}\right) \Pi_{n-1}\right)^{-1}+\tilde{\alpha} \tilde{T}_{n} \mathbf{e}_{n}^{t}\right)\right)
\end{array}
$$

If we choose $\alpha \tilde{\alpha}=\frac{1}{J_{n}}$, we have:

$$
\begin{array}{ll} 
& \frac{1}{J_{n} \psi_{n} \phi_{n}}\left(W_{n+1}(x, y)-W_{n}(x, y)\right) \\
= & \operatorname{det}_{n+1}\left(\operatorname{Id}_{n+1}+\left(\left(\Pi_{n-1}(x-Q) \Pi_{n-1}\right)^{-1}+\alpha \mathbf{e}_{n} T_{n}^{t}\right)\left(\left(\Pi_{n-1}\left(y-P^{t}\right) \Pi_{n-1}\right)^{-1}\right.\right. \\
& \left.\left.+\tilde{\alpha} \tilde{T}_{n} \mathbf{e}_{n}^{t}\right)\right) \\
= & \operatorname{det}_{n}\left(\operatorname{Id}_{n}+\left(\left(\Pi_{n-1}\left(y-P^{t}\right) \Pi_{n-1}\right)^{-1}+\tilde{\alpha} \tilde{T}_{n} \mathbf{e}_{n}^{t}\right)\left(\left(\Pi_{n-1}(x-Q) \Pi_{n-1}\right)^{-1}\right.\right. \\
& \left.\left.\quad+\alpha \mathbf{e}_{n} T_{n}^{t}\right)\right) \\
& \quad \operatorname{det}_{n}\left(\operatorname{Id}_{n}+\left(\Pi_{n-1}\left(y-P^{t}\right) \Pi_{n-1}\right)^{-1}\left(\Pi_{n-1}(x-Q) \Pi_{n-1}\right)^{-1}+\frac{1}{J_{n}} \tilde{T}_{n} T_{n}^{t}\right)
\end{array}
$$

$(5-10)$

Then, we multiply both sides by Eq. (5-1), i.e. by $\operatorname{det}\left(\Pi_{n-1}(x-Q) \Pi_{n-1}\right)$ and $\operatorname{det}\left(\Pi_{n-1}\left(y-P^{t}\right) \Pi_{n-1}\right)$ :

$$
\begin{aligned}
& \frac{h_{n} \mathrm{e}^{V_{1}+V_{2}}}{J_{n}}\left(W_{n+1}(x, y)-W_{n}(x, y)\right) \\
& =\quad \operatorname{det}_{n}\left(\operatorname{Id}_{n}+\Pi_{n-1}\left(y-P^{t}\right) \Pi_{n-1}(x-Q) \Pi_{n-1}\right. \\
& \left.+\frac{1}{J_{n}} \Pi_{n-1}\left(y-P^{t}\right) \tilde{T}_{n} T_{n}^{t}(x-Q) \Pi_{n-1}\right)
\end{aligned}
$$

We have:

$$
\begin{aligned}
T_{n}^{t}(x-Q) \Pi_{n-1} & =\frac{1}{\psi_{n}} \mathbf{e}_{n}^{t} R(x-Q) \Pi_{n-1}+\hat{\phi}_{\infty}^{t} \Pi_{n-1}(x-Q) \Pi_{n-1} \\
& =\quad \frac{\psi_{n}}{\mathbf{\psi}_{n}^{t}}\left(1-\frac{\psi_{\infty} \mathbf{e}_{0}^{t}}{\psi_{0}}\right) \Pi_{n-1}+\hat{\phi}_{\infty}^{t}(x-Q)-\hat{\phi_{\infty}}{ }^{t} \Pi^{n}(x-Q) \Pi_{n-1} \\
& =-\frac{\mathbf{e}_{0}^{t}}{\psi_{0}}+\frac{\mathbf{e}_{0}^{t}}{\psi_{0}}-\hat{\phi_{\infty}^{t}} \Pi^{n}(x-Q) \Pi_{n-1} \\
& =-\hat{\phi}_{\infty}^{t} \Pi^{n}(x-Q) \Pi_{n-1} \\
(5-12) &
\end{aligned}
$$

Notice also that Heisenberg's relation Eq. (2-45) implies:

$$
\operatorname{Id}_{n}+\Pi_{n-1}\left(y-P^{t}\right)(x-Q) \Pi_{n-1}=\Pi_{n-1}(x-Q)\left(y-P^{t}\right) \Pi_{n-1}
$$


Thus:

$$
\begin{aligned}
& \frac{h_{n} \mathrm{e}^{V_{1}+V_{2}}}{J_{n}}\left(W_{n+1}(x, y)-W_{n}(x, y)\right) \\
= & \operatorname{det}_{n}\left(\operatorname{Id}_{n}+\Pi_{n-1}\left(y-P^{t}\right) \Pi_{n-1}(x-Q) \Pi_{n-1}\right. \\
& \left.\quad+\frac{1}{J_{n}} \Pi_{n-1}\left(y-P^{t}\right) \Pi^{n}{\hat{\psi_{\infty}}}_{\hat{\infty}}^{t} \Pi^{n}(x-Q) \Pi_{n-1}\right) \\
= & \operatorname{det}_{n}\left(\operatorname{Id}_{n}+\Pi_{n-1}\left(y-P^{t}\right)(x-Q) \Pi_{n-1}-\Pi_{n-1}\left(y-P^{t}\right) \Pi^{n}(x-Q) \Pi_{n-1}\right. \\
& \left.\quad+\frac{1}{J_{n}} \Pi_{n-1}\left(y-P^{t}\right) \Pi^{n} \hat{\psi_{\infty}}{\hat{\phi_{\infty}}}^{t} \Pi^{n}(x-Q) \Pi_{n-1}\right) \\
= & \operatorname{det}_{n}\left(\Pi_{n-1}(x-Q)\left(y-P^{t}\right) \Pi_{n-1}-\Pi_{n-1}\left(y-P^{t}\right) \Pi^{n}\left(1-\frac{\hat{\psi_{\infty}}}{J_{\infty}}\right) \Pi^{n}(x-Q) \Pi_{n-1}\right)
\end{aligned}
$$

Now, use Eq. (5-4) and Eq. (5-8), that gives:

$$
\begin{aligned}
& \quad \frac{1}{J_{n} K_{n+1}}\left(W_{n+1}(x, y)-W_{n}(x, y)\right) \\
& =\quad \operatorname{det}_{n}\left(\operatorname{Id}_{n}-\Pi_{n-1}\left(y-P^{t}\right) \Pi^{n}\left(1-\frac{\hat{\psi_{\infty} \hat{\phi}_{\infty}}}{J_{n}}\right) \Pi^{n}(x-Q) \Pi_{n-1} \tilde{R}^{t} \times\right. \\
& \left.\quad \Pi_{n}\left(1-\frac{\psi_{\infty} \phi_{\infty}^{t}}{K_{n+1}}\right) \Pi_{n} R \Pi_{n-1}\right) \\
& =\quad \operatorname{det}_{n+1}\left(\operatorname{Id}_{n+1}-\Pi_{n}\left(1-\frac{\psi_{\infty} \phi_{\infty}^{t}}{K_{n+1}}\right) \Pi_{n} R \Pi_{n-1}\left(y-P^{t}\right) \Pi^{n}\left(1-\frac{\hat{\psi_{\infty}}{\hat{\phi_{\infty}}}^{t}}{J_{n}}\right) \times\right. \\
& \left.\quad \Pi^{n}(x-Q) \Pi_{n-1} \tilde{R}^{t} \Pi_{n}\right) \\
& (5-15) \quad
\end{aligned}
$$

Now, because of Eq. (2-47), we have:

$$
\begin{array}{rlrl}
\Pi_{n} R \Pi_{n-1}\left(y-P^{t}\right) \Pi^{n} & = & \Pi_{n} R V_{1}^{\prime}(Q) \Pi^{n} \\
& = & -\Pi_{n} \frac{V_{1}^{\prime}(x)-V_{1}^{\prime}(Q)}{x-Q} \Pi^{n} \\
& = & -\mathcal{W}_{n}(x) & \\
(5-16) & &
\end{array}
$$

Therefore:

$$
W_{n+1}(x, y)-W_{n}(x, y)=J_{n} K_{n+1} \operatorname{det}_{n+1}\left(\operatorname{Id}_{n+1}-\Pi_{n}\left(1-\frac{\psi_{\infty} \phi_{\infty}^{t}}{K_{n+1}}\right) \mathcal{W}_{n}\left(1-\frac{\hat{\psi_{\infty} \hat{\phi}_{\infty}}}{J_{n}}\right) \tilde{\mathcal{W}}_{n}^{t}\right)
$$

The second part of the formula is obtained by applying Lemma $\amalg .2$ 


\section{Appendix F Proof of theorem 8.1}

We start from theorem 7.1 ;

$$
\begin{aligned}
& W_{n}(x, y)= \gamma_{n}^{2} \operatorname{det}\left(\mathrm{Id}_{n+1}-U_{n}(x, y) \tilde{U}_{n}(x, y)^{t}\right) \\
&\left(\left(J_{n+d_{2}}+\hat{\vec{\phi}}_{n} \frac{1}{1-\tilde{U}_{n}^{t} U_{n}} \hat{\vec{\psi}}\right)\left(K_{n-d_{2}}+\vec{\phi}_{n}^{t} \frac{1}{1-U_{n} \tilde{U}_{n}^{t}} \vec{\psi}_{n}\right)\right. \\
&\left.-\left(\hat{\vec{\phi}}_{n}^{t} \tilde{U}_{n}^{t} \frac{1}{1-U_{n} \tilde{U}_{n}^{t}} \vec{\psi}_{n}\right)\left(\vec{\phi}_{n}^{t} \frac{1}{1-U_{n} \tilde{U}_{n}^{t}} U_{n} \hat{\vec{\psi}}_{n}\right)\right)
\end{aligned}
$$

Let us compute the various terms:

$$
\begin{aligned}
& \vec{\phi}_{n}^{t} \frac{1}{1-U_{n} \tilde{U}_{n}^{t}} \vec{\psi}_{n} \\
= & \vec{\phi}_{n}^{t} \frac{1}{1-U_{n} \tilde{U}_{n}^{t}} \vec{\psi}_{n} \\
= & \vec{\phi}_{n}^{t} \tilde{U}_{n}^{t-1} A_{n} \Psi_{n} \frac{1}{\left(\tilde{U}_{n}^{t-1}-U_{n}\right) A_{n} \Psi_{n}} \vec{\psi}_{n} \\
= & -\phi_{\infty}^{t} B_{n-d_{2}}^{t} \Pi_{n-1} \Psi_{\infty}(x) \frac{1}{y-\Psi_{n}^{-1} \Psi_{n}^{\prime}} f^{(0)} \\
= & -\phi_{\infty}^{t}(y) \Pi_{n-d_{2}} B_{n-d_{2}}^{t} \Pi_{n-1} \Psi_{\infty}(x) \frac{1}{y-\Psi_{n}^{-1}(x) \Psi_{n}^{\prime}(x)} f^{(0)} \\
= & \mathrm{e}^{-V_{2}(y)} O\left(y^{n-d_{2}-1}\right)
\end{aligned}
$$

where $f^{(0)}$ is the vector $(1,0, \ldots, 0)^{t}$ of dimension $d_{2}+1$.

At large $y$, this expression behaves like $\mathrm{e}^{-V_{2}(y)} O\left(y^{n-d_{2}-1}\right)$.

$$
\begin{aligned}
& \hat{\phi}_{\infty}^{t} \Pi_{n+d_{2}-1}^{n-1} \frac{1}{1-\tilde{U}_{n}^{t} U_{n}} \Pi_{n+d_{1}-1}^{n-1} \hat{\psi_{\infty}} \\
= & f^{(0) t} \hat{\Phi}_{n}^{t} \frac{1}{\tilde{U}_{n}^{t-1}-U_{n}} \tilde{U}_{n}^{t-1} \hat{\Psi}_{n} f^{(0)} \\
= & f^{(0) t} \hat{\Phi}_{n}^{t} A_{n} \Psi_{n} \frac{1}{\left(\tilde{U}_{n}^{t-1}-U_{n}\right) A_{n} \Psi_{n}} \tilde{U}_{n}^{t-1} \hat{\Psi}_{n} f^{(0)} \\
= & f^{(0) t} \frac{1}{y \Psi_{n}-\Psi_{n}^{\prime}} \tilde{U}_{n}^{t-1} \hat{\Psi}_{n} f^{(0)} \\
= & f^{(0) t} \frac{1}{y-\Psi_{n}^{-1} \Psi_{n}^{\prime}} \Psi_{n}^{-1} \tilde{U}_{n}^{t-1} \hat{\Psi}_{n} f^{(0)} \\
= & f^{(0) t} \frac{1}{y-\Psi_{n}^{-1} \Psi_{n}^{\prime}} \hat{\Phi}_{n}^{t} A_{n} \tilde{U}_{n}^{t-1} \hat{\Psi}_{n} f^{(0)} \\
= & -f^{(0) t} \frac{1}{y-\Psi_{n}^{-1}(x) \Psi_{n}^{\prime}(x)} \hat{\Phi}_{\infty}^{t}(x) \Pi^{n} B_{n+d_{2}}^{t} \hat{\vec{\psi}}(y) \\
= & \mathrm{e}^{V_{2}(y)} \frac{O\left(y^{-n-d_{2}-1}\right)}{}
\end{aligned}
$$

It behaves as $\mathrm{e}^{V_{2}(y)} O\left(y^{-n-d_{2}-1}\right)$ at large $y$. 


$$
\begin{aligned}
& {\hat{\phi_{\infty}}}^{t} \tilde{U}_{n}^{t} \frac{1}{1-U_{n} \tilde{U}_{n}^{t}} \psi_{\infty} \\
= & f^{(0) t} \hat{\Phi}_{n}^{t} \frac{1}{\tilde{U}_{n}^{t-1}-U_{n}} \Psi_{n} f^{(0)} \\
= & f^{(0) t} \hat{\Phi}_{n}^{t} A_{n} \Psi_{n} \frac{1}{\left(\tilde{U}_{n}^{t-1}-U_{n}\right) A_{n} \Psi_{n}} \Psi_{n} f^{(0)} \\
= & f^{(0) t} \frac{1}{y \Psi_{n}-\Psi_{n}^{\prime}} \Psi_{n} f^{(0)} \\
= & f^{(0) t} \frac{1}{y-\Psi_{n}^{-1} \Psi_{n}^{\prime}} f^{(0)} \\
= & \left(\frac{1}{y-H_{n}(x, x)}\right)^{(00)}
\end{aligned}
$$

- Similarly, we have:

$$
\phi_{\infty}^{t} \frac{1}{1-U_{n} \tilde{U}_{n}^{t}} U_{n} \hat{\psi_{\infty}}=\left(\frac{1}{x-\tilde{H}_{n}(y, y)}\right)^{(00)}
$$

but unfortunately, that formula is not sufficient to find the large $y$ behaviour.

Instead, we prove the following lemma:

\section{Lemma F.1}

$$
\left(x+V_{2}^{\prime}(y)\right) \phi_{\infty}^{t} \frac{1}{1-U_{n} \tilde{U}_{n}^{t}} U_{n} \hat{\psi}_{n}=-1-\frac{n}{\tilde{t} y^{d_{2}+1}}+O\left(y^{-d_{2}-2}\right)
$$

and symetricaly:

$$
\left(y+V_{1}^{\prime}(x)\right) \hat{\phi}_{\infty}^{t} \tilde{U}_{n}^{t} \frac{1}{1-U_{n} \tilde{U}_{n}^{t}} \psi_{n}=-1-\frac{n}{t x^{d_{1}+1}}+O\left(x^{-d_{1}-2}\right)
$$

\section{proof:}

Compute the large $y$ behaviour of the following matrix:

$$
\begin{aligned}
& \Pi^{n-1}\left(\tilde{U}_{n}^{t}+\left(x+V_{2}^{\prime}\right) \hat{\psi}_{n} \phi_{\infty}{ }^{t}\right) \Pi_{n} \\
= & \Pi^{n-1}\left(\tilde{R}^{t}(x-Q)+\left(x+V_{2}^{\prime}\right) \hat{\psi}_{n} \phi_{\infty}{ }^{t}\right) \Pi_{n} \\
= & \Pi^{n-1}\left(\left(\tilde{R}^{t}+\hat{\psi}_{\infty} \phi_{\infty}{ }^{t}\right)(x-Q)+\hat{\psi}_{n} \phi_{\infty}{ }^{t}\left(V_{2}^{\prime}(y)-V_{2}^{\prime}\left(P^{t}\right)\right)+\hat{\psi}_{n} \phi_{\infty}{ }^{t}\left(V_{2}^{\prime}\left(P^{t}\right)+Q\right)\right) \Pi_{n} \\
= & \Pi^{n-1}\left(\frac{1}{y-P^{t}}(x-Q)+\hat{\psi}_{n} \phi_{\infty}{ }^{t}\left(V_{2}^{\prime}\left(P^{t}\right)+Q\right)\right) \Pi_{n}
\end{aligned}
$$

It is easy to see that this expression behaves like $O\left(y^{-1}\right)$ at large $y$. The leading term in $y$ is:

$$
\frac{1}{y} \Pi^{n-1}(x-Q) \Pi_{n}+\hat{\psi}_{n-1} \mathrm{e}^{-V_{2}(y)} \frac{\partial}{\partial y}\left(\mathrm{e}^{V_{2}(y)} \phi_{n}(y)\right) \mathbf{e}_{n-1} \mathbf{e}_{n}^{t}+O\left(y^{-2}\right)
$$




$$
\begin{aligned}
& =\frac{1}{y} \Pi^{n-1}(x-Q) \Pi_{n}+\sqrt{h_{n-1}} y^{-n} \frac{n}{\sqrt{h_{n}}} y^{n-1} \mathbf{e}_{n-1} \mathbf{e}_{n}^{t}+O\left(y^{-2}\right) \\
& =\frac{1}{y}\left(\Pi^{n-1}(x-Q) \Pi_{n}+\frac{n}{\gamma_{n}} \mathbf{e}_{n-1} \mathbf{e}_{n}^{t}\right)+O\left(y^{-2}\right) \\
(6-9) &
\end{aligned}
$$

and thus, using $U_{n}=-\frac{y}{\gamma_{n}} \mathbf{e}_{n} \mathbf{e}_{n-1}^{t}+O(1)$, we have:

$$
\begin{array}{ll} 
& U_{n}\left(\tilde{U}_{n}^{t}+\left(x+V_{2}^{\prime}\right) \hat{\psi}_{n} \phi_{\infty}{ }^{t}\right) \Pi_{n} \\
= & -\frac{1}{\gamma_{n}} \mathbf{e}_{n} \mathbf{e}_{n-1}^{t}\left((x-Q) \Pi_{n}+\frac{n}{\gamma_{n}} \mathbf{e}_{n-1} \mathbf{e}_{n}^{t}\right)+O\left(y^{-1}\right) \\
= & -\frac{1}{\gamma_{n}} \mathbf{e}_{n}\left(\mathbf{e}_{n-1}^{t}(x-Q) \Pi_{n}+\frac{n}{\gamma_{n}} \mathbf{e}_{n}^{t}\right)+O\left(y^{-1}\right)
\end{array}
$$$$
(6-10)
$$

and the following determinant is:

$$
\begin{aligned}
& \operatorname{det}\left(1-U_{n}\left(\tilde{U}_{n}^{t}+\left(x+V_{2}^{\prime}\right) \hat{\psi}_{n} \phi_{\infty}{ }^{t}\right) \Pi_{n}\right) \\
= & \operatorname{det}\left(1+\frac{1}{\gamma_{n}} \mathbf{e}_{n}\left(\mathbf{e}_{n-1}^{t}(x-Q) \Pi_{n}+\frac{n}{\gamma_{n}} \mathbf{e}_{n}^{t}\right)+O\left(y^{-1}\right)\right) \\
= & \left.1+\frac{1}{\gamma_{n}}\left(\mathbf{e}_{n-1}^{t}(x-Q) \mathbf{e}_{n}+\frac{n}{\gamma_{n}}\right)+O\left(y^{-1}\right)\right) \\
= & \left.1+\left(\frac{n}{\gamma_{n}^{2}}-1\right)+O\left(y^{-1}\right)\right) \\
= & \left.\frac{n}{\gamma_{n}^{2}}+O\left(y^{-1}\right)\right)
\end{aligned}
$$

It follows:

$$
\begin{aligned}
& \left.\frac{n}{\gamma_{n}^{2}}+O\left(y^{-1}\right)\right) \\
= & \operatorname{det}\left(1-U_{n}\left(\tilde{U}_{n}^{t}+\left(x+V_{2}^{\prime}\right) \hat{\psi}_{n} \phi_{\infty}^{t}\right) \Pi_{n}\right) \\
= & \left.\operatorname{det}\left(1-U_{n} \tilde{U}_{n}^{t}+\left(x+V_{2}^{\prime}\right) U_{n} \hat{\psi}_{n} \phi_{\infty}^{t}\right) \Pi_{n}\right) \\
= & \left.\operatorname{det}\left(1-U_{n} \tilde{U}_{n}^{t}\right) \operatorname{det}\left(1+\left(x+V_{2}^{\prime}\right) \frac{1}{1-U_{n} \tilde{U}_{n}^{t}} U_{n} \hat{\psi}_{n} \phi_{\infty}^{t}\right) \Pi_{n}\right) \\
= & \operatorname{det}\left(1-U_{n} \tilde{U}_{n}^{t}\right)\left(1+\left(x+V_{2}^{\prime}\right) \phi_{\infty}^{t} \frac{1}{1-U_{n} \tilde{U}_{n}^{t}} U_{n} \hat{\psi}_{n}\right)
\end{aligned}
$$

that implies:

$$
\begin{aligned}
\left(x+V_{2}^{\prime}\right) \phi_{\infty}{ }^{t} \frac{1}{1-U_{n} \tilde{U}_{n}^{t}} U_{n} \hat{\psi}_{n}= & -1+\frac{n}{\gamma_{n}^{2} \operatorname{det}\left(1-U_{n} \tilde{U}_{n}^{t}\right)}+O\left(y^{-d_{2}-2}\right) \\
& =-1-\frac{n}{\mathcal{E}_{n}(x, y)}+O\left(y^{-d_{2}-2}\right) \\
& =-1-\frac{n}{\tilde{t} y^{d_{2}+1}}+O\left(y^{-d_{2}-2}\right)
\end{aligned}
$$


similarly we have:

$$
\left(y+V_{1}^{\prime}(x)\right) \hat{\phi}_{\infty}^{t} \tilde{U}_{n}^{t} \frac{1}{1-U_{n} \tilde{U}_{n}^{t}} \psi_{n}=-1-\frac{n}{t x^{d_{1}+1}}+O\left(x^{-d_{2}-2}\right)
$$

\section{F.5 U-conjecture, Lemma 8.1}

Using lemma F.1, we have:

$$
\begin{aligned}
& \left(x+V_{2}^{\prime}(y)\right) \operatorname{det}\left(1-U_{n} \tilde{U}_{n}^{t}\right)\left(\hat{\phi}_{\infty}^{t} \tilde{U}_{n}^{t} \frac{1}{1-U_{n} \tilde{U}_{n}^{t}} \psi_{n}\right)\left(\phi_{\infty}^{t} \frac{1}{1-U_{n} \tilde{U}_{n}^{t}} U_{n} \hat{\psi}_{n}\right) \\
& =\quad-\operatorname{det}\left(1-U_{n} \tilde{U}_{n}^{t}\right)\left(\hat{\phi}_{\infty}^{t} \tilde{U}_{n}^{t} \frac{1}{1-U_{n} \tilde{U}_{n}^{t}} \psi_{n}\right)-\frac{n}{\gamma_{n}^{2}}\left(\hat{\phi}_{\infty}^{t} \tilde{U}_{n}^{t} \frac{1}{1-U_{n} \tilde{U}_{n}^{t}} \psi_{n}\right) \\
& +O\left(y^{-2}\right) \\
& =\quad-\operatorname{det}\left(1-U_{n} \tilde{U}_{n}^{t}\right)\left(\frac{1}{y-H_{n}(x, x)}\right)^{(00)}-\frac{n}{\gamma_{n}^{2}}\left(\frac{1}{y-H_{n}(x, x)}\right)^{(00)} \\
& +O\left(y^{-2}\right) \\
& =\frac{\tilde{t}}{\gamma_{n}^{2}} \operatorname{det}\left(y-H_{n}(x, x)\right)\left(\frac{1}{y-H_{n}(x, x)}\right)^{(00)}-\frac{n}{\gamma_{n}^{2}}\left(\frac{1}{y-H_{n}(x, x)}\right)^{(00)} \\
& +O\left(y^{-2}\right) \\
& =\frac{\tilde{t}}{\gamma_{n}^{2}} \operatorname{det}\left(y-H_{n}(x, x)\right)\left(\frac{1}{y-H_{n}(x, x)}\right)^{(00)}+O\left(y^{-1}\right)
\end{aligned}
$$

The first term is a polynomial in $y$, and this proves theorem 8.1

\section{Appendix G Proof of theorem 8.3}

Starting from lemma F.1, we have:

$$
\begin{aligned}
& \gamma_{n}^{2} \operatorname{det}\left(1-U_{n} \tilde{U}_{n}^{t}\right)\left(y+V_{1}^{\prime}(x)\right)\left(\hat{\phi_{\infty}}{ }^{t} \tilde{U}_{n}^{t} \frac{1}{1-U_{n} \tilde{U}_{n}^{t}} \psi_{n}\right) \\
= & \mathcal{E}_{n}(x, y)\left(1+\frac{n}{t x^{d_{1}+1}}\right)+O\left(x^{-1}\right) \\
= & \mathcal{E}_{n}(x, y)+n+O\left(x^{-1}\right) \\
(7-1) &
\end{aligned}
$$

from which theorem 8.3 follows. 


\section{Appendix $\mathrm{H} \quad$ Proof of theorem 8.2}

Let $r_{n}(x)$ be the matrix:

$$
r_{n}(x)=\Pi_{n+1}^{n-d_{2}+1} F_{n}(x)=\Pi_{n+1}^{n-d_{2}+1}+\frac{1}{\gamma_{n+1}} \mathbf{e}_{n+1} \mathbf{e}_{n}^{t}(x-Q)
$$

By definition, it is such that:

$$
\Psi_{n+1}(x)=r_{n}(x) \Psi_{n}(x)
$$

Thus:

$$
\begin{array}{rll}
\mathcal{D}_{n+1}(x) & =\quad \Psi_{n+1}^{\prime} \Psi_{n+1}^{-1}=\left(r_{n} \Psi_{n}\right)^{\prime}\left(r_{n} \Psi_{n}\right)^{-1} \\
& =\quad r_{n}^{\prime} r_{n}^{-1}+r_{n} \mathcal{D}_{n} r_{n}^{-1} \\
& =\quad r_{n}\left(r_{n}^{-1} r_{n}^{\prime}+\mathcal{D}_{n}\right) r_{n}^{-1} \\
(8-3) &
\end{array}
$$

Therefore:

$$
\mathcal{E}_{n+1}=\tilde{t} \operatorname{det}\left(y-\mathcal{D}_{n}-r_{n}^{-1} r_{n}^{\prime}\right)
$$

Now, notice that $r_{n}^{-1} r_{n}^{\prime}=-\frac{1}{Q_{n, n-d_{2}}} \mathbf{e}_{n-d_{2}} \mathbf{e}_{n}^{t}$, thus

$$
\mathcal{E}_{n+1}-\mathcal{E}_{n}=(-1)^{d_{2}+1} \frac{\tilde{t}}{Q_{n, n-d_{2}}} \operatorname{det}\left(\mathcal{C}_{n}\right)
$$

where we have defined

$$
\mathcal{C}_{n}(x, y)=\Pi_{n}^{n-d_{2}+1}\left(y+\mathcal{D}_{n}(x)\right) \Pi_{n-1}^{n-d_{2}}
$$

We have:

$$
\begin{array}{rll}
\mathcal{C}_{n} & =\Pi_{n}^{n-d_{2}+1}\left(y-P^{t}\right) \Pi_{n-1}^{n-d_{2}}+\Pi_{n}^{n-d_{2}+1} \frac{V_{1}^{\prime}(x)-V_{1}^{\prime}(Q)}{x-Q}\left(1-\Pi_{n-1}\right)(x-Q) \Pi_{n-1}^{n-d_{2}} \\
& =\quad \Pi_{n}^{n-d_{2}+1}\left(y-P^{t}\right) \Pi_{n-1}^{n-d_{2}}+\Pi_{n}^{n-d_{2}+1} \mathcal{W}_{n}(x-Q) \Pi_{n-1}^{n-d_{2}} \\
(8-7) & &
\end{array}
$$

and:

$$
\mathcal{C}_{n} \Pi_{n-1}^{n-d_{2}} \tilde{R}^{t} \Pi_{n}^{n-d_{2}+1}=\Pi_{n}^{n-d_{2}+1}\left(1-\mathcal{W}_{n} \tilde{\mathcal{W}}_{n}^{t}\right) \Pi_{n}^{n-d_{2}+1}
$$

That implies:

$$
\begin{aligned}
\operatorname{det}\left(\mathcal{C}_{n}\right) \Pi_{n-1}^{n-d_{2}} \tilde{R}^{t} \Pi_{n}^{n-d_{2}+1} & =(-1)^{d_{2}} \gamma_{n} \ldots \gamma_{n-d_{2}+1} \operatorname{det}\left(1-\mathcal{W}_{n} \tilde{\mathcal{W}}_{n}^{t}\right) \\
& =(-1)^{d_{2}+1} \frac{Q_{n, n-d_{2}}}{\tilde{t}} \operatorname{det}\left(1-\mathcal{W}_{n} \tilde{\mathcal{W}}_{n}^{t}\right)
\end{aligned}
$$$$
(8-9)
$$

which proves the formula. 


\section{Appendix I A usefull formula for determinants with rank 2 matrices}

Lemma I.2 if $M$ is an invertible matrix, and $a, b, c, d$ are arbitrary vectors, we have:

$$
\operatorname{det}\left(M+a b^{t}+c d^{t}\right)=\operatorname{det} M\left(\left(1+b^{t} \frac{1}{M} a\right)\left(1+d^{t} \frac{1}{M} c\right)-b^{t} \frac{1}{M} c d^{t} \frac{1}{M} a\right)
$$

\section{References}

[1] G. Akemann, A. Pottier, J. Phys. A: Math Gen 37 L453-60, hep-th/0404068.

[2] M.C. Bergère, "Orthogonal Polynomials for Potentials of two Variables with External Sources", hep-th/0311227.

[3] M.C. Bergère, "Biorthogonal Polynomials for Potentials of two Variables and External Sources at the Denominator", hep-th/0404126.

[4] M.C. Bergère, "Correlation Functions of Complex Matrix Models", J. Phys. A: Math. Gen 39 (2006), hep-th/0511019.

[5] M. Bertola, Conjecture for the spectral curve of biorthogonal differential systems, private communication (2003).

[6] M. Bertola, "Bilinear semi-classical moment functionals and their integral representation", J. App. Th. 121 71-99 (2003).

[7] M. Bertola, M. Y. Mo, "Isomonodromic deformation of resonant rational connections", nlin.SI/0510011.

[8] M. Bertola, B. Eynard, "Mixed Correlation Functions of the Two-Matrix Model", J. Phys. A36 (2003) 7733-7750, hep-th/0303161.

[9] M. Bertola, B. Eynard, J. Harnad, "Duality, Biorthogonal Polynomials and MultiMatrix Models", Commun. Math. Phys. 229 73-120 (2002).

[10] M. Bertola, B. Eynard, J. Harnad, "Differential systems for biorthogonal polynomials appearing in 2-matrix models and the associated Riemann-Hilbert problem", CRM-2852, Saclay-T02/097, nlin.SI/0208002, to appear in Commun. Math. Phys. in press (2002). 
[11] M. Bertola, B. Eynard, "The PDEs of biorthogonal polynomials arising in the 2matrix model", Math. Phys., Analysis and Geometry, ISSN: 1385-0172, 1572-9656. nlin.SI/0311033.

[12] M. Bertola, B. Eynard, J. Harnad, "Partition functions for matrix models and isomonodromic tau functions", J. Phys. A., 36 (2003) 3067-3083.

[13] M. Bertola, J. Harnad, A. Its, "Dual Riemann Hilbert approach to biorthogonal polynomials", in preparation.

[14] L. Chekhov, B. Eynard, N. Orantin, "Free energy topological expansion for the 2-matrix model", math-ph/0603003.

[15] P. Di Francesco, P. Ginsparg, J. Zinn-Justin, "2D Gravity and Random Matrices", Phys. Rep. 254, 1 (1995).

[16] B. Eynard, M.L. Mehta, "Matrices coupled in a chain: eigenvalue correlations", J. Phys. A: Math. Gen. 31, 4449 (1998), cond-mat/9710230.

[17] B. Eynard, "Eigenvalue distribution of large random matrices, from one matrix to several coupled matrices", Nuc. Phys. B 506,3 633-664 (1997).

[18] B. Eynard, "The 2-matrix model, biorthogonal polynomials, Riemann-Hilbert problem, and algebraic geometry", Habilitation thesis, Paris VII university, in french, math-ph/0504034.

[19] B. Eynard, "A short note about Morozov's formula", math-ph/0406063.

[20] B. Eynard, N. Orantin, "Mixed correlation functions in the 2-matrix model, and the Bethe ansatz", JHEP/0508 (2005) 028, hep-th/0504029.

[21] B. Eynard, A. Prats Ferrer, "2-matrix versus complex matrix model, integrals over the unitary group as triangular integrals", hep-th/0502041, accepted in Communications in Mathematical physics.

[22] A. Fokas, A. Its, A. Kitaev, "The isomonodromy approach to matrix models in 2D quantum gravity", Commun. Math. Phys. 147, 395-430 (1992).

[23] Y. V. Fyodorov, E. Strahov, "An exact formula for general spectral correlation function of random Hermitian matrices", J. PHYS. A 363203 (2003).

[24] J. Ginibre, "Statistical ensembles of complex, quaternion and real matrices", $J$. Math. Phys. 61965440. 
[25] J. Harnad, "Two matrix models and biorthogonal polynomials, integral representations of fundamental systems", CRM Short Program on Random Matrices, Random Processes and Integrable Systems 20 June - 8 July 2005, http://www.crm.umontreal.ca/

[26] J. Hurtubise, Some formula for the spectral curve of biorthogonal differential systems, private communication (2001).

[27] M. Jimbo, T. Miwa and K. Ueno, "Monodromy Preserving Deformation of Linear Ordinary Differential Equations with Rational Coeefficients I.", Physica 2D, 306352 (1981).

[28] M. Jimbo and T. Miwa, "Monodromy Preserving Deformation of Linear Ordinary Differential Equations with Rational Coeefficients II, III", Physica 2D, 407-448 (1981); ibid., 4D, 26-46 (1981).

[29] A. A. Kapaev, "The Riemann-Hilbert problem for the bi-orthogonal polynomials", J.Phys. A 36 (2003) 4629-4640.

[30] V.A. Kazakov, "Ising model on a dynamical planar random lattice: exact solution", Phys Lett. A119, 140-144 (1986).

[31] C. Kristjansen, J. Plefka, G. W. Semenoff and M. Staudacher, "A new doublescaling limit of $\mathrm{N}=4$ super Yang-Mills theory and PP-wave strings, Nucl. Phys. B, to appear (2002), hep-th/0205033.

[32] A. Kuijlars, K. T-R McLaughlin, "A Riemann-Hilbert problem for biorthogonal polynomials", J. Comput. Appl. Math. 178 (2005), 313-320. math.CV/0310204.

[33] M.L. Mehta, "A method of integration over matrix variables", Commun. Math. Phys. 79, 327 (1981).

[34] M.L. Mehta, Random Matrices, 2nd edition, (Academic Press, New York, 1991).

[35] A. Morozov, "Pair correlator in the Itzykson-Zuber Integral", Modern Phys. Lett. A 7, no. 37 3503-3507 (1992).

[36] K. Ueno and K. Takasaki, "Toda Lattice Hierarchy", Adv. Studies Pure Math. 4, 1-95 (1984).

[37] V. B. Uvarov, 1969 USSR, Comput. Math. 9 25-36.

[38] P. Wiegmann, A. Zabrodin, "Large N expansion for normal and complex matrix ensembles", hep-th/0309253. 